\title{
Potential Severe Effects of a Biosphere Collision and Planetary Protection Implications
}

Corresponding author: Robert Walker (robert@robertinventor.com)

Preprint DOI

\subsection{9/osf.io/kad38}

\section{Contents}

Contents

Abstract $\quad 4$

$\begin{array}{ll}\text { Extended abstract } & 4\end{array}$

Introduction $\quad 8$

$\begin{array}{ll}\text { Planetary protection measures under scrutiny } & 15\end{array}$

Example of early life on Mars shows potential for complete extinction in forward direction

Could evolution on Mars evolve as fast as on Earth, reaching simple animals such as sponges, evolve faster, reaching a genetic complexity we can't yet match?

Potential Mars surface and near surface habitats

Potential for fresh liquid water in polar regions through the solid state greenhouse effect - of special planetary protection relevance

Potential deep subsurface habitats communicating with the surface including ice fumaroles and hydrothermal systems on Mars

Lichens, cyanobacteria and black yeasts surviving in modern Mars surface conditions (similar to Gale crater) and other examples of Earth life that could potentially survive on Mars

Microhabitats - such as micropores in salt pillars and ground hugging water vapour as morning frosts evaporate

Unanswered questions about Mars special regions

Modern Mars habitability reassessment 
Radioresistant extremophiles like Chroococcidiopsis

UV tolerance by extremophiles and transport of spores in martian dust storms

Sources of nitrogen on Mars

All conditions for life may be present on or near the modern Mars surface

Could complete extinction be possible in the backwards direction, from space to Earth?

The need for a vigorous in situ astrobiological search

List of astrobiological instruments for in situ searches

Well beyond the capabilities of currently planned rovers - preparation for humans on Mars prioritized over astrobiology

Top candidate sites for an astrobiological investigation of Mars

Candidate sites for past life

Is transfer of life possible from Mars to Earth?

Has life from Mars caused mass extinctions on Earth in the past? Was the Great Oxygenation Event such an extinction?

Transfer of life is hard in the direction from Earth to Mars - and especially so for photosynthetic life (MID EDIT)

Accidental similarity of amino acids

Exotoxins, protoxins, allergens and opportunistic infection

Chemical transformation of habitats by life

Global modification - is a martian Gaia possible and could anti-Gaia lead to a martian swansong biosphere? - NEEDS CITES

Global impacts or minor nuisances in backwards direction to Earth

Potential for a more efficient photosynthetic life form based on exobiology - example of a global impact

Impossibility of quarantine provisions until you can assess the risk

Legal and practical issues for sample returns - a likely minimum of 20 years from initiation of legal procedures to permission for launch of the sample return mission 73

A method for $100 \%$ safety for sample returns without the legal complexity or the issues of building the receiving facility

Assessing the risk - Drake type equation approach

Mathematics of backwards contamination

Drake type equation approach to extinctions in the forward direction 
New version of the precautionary principle for superpositive outcomes

Mundane value of astrobiology for business - example of the billion dollar extremophile enzymes industry

Are $100 \%$ sterile rovers and instruments possible in the forward direction?

Designs for $500{ }^{\circ} \mathrm{C}$ to decompose organics if higher temperatures than $300{ }^{\circ} \mathrm{C}$ are needed

$\mathrm{CO}_{2}$ snow sterilization - alternative to temperatures high enough to decompose organics - MID EDIT

Sterilizing in situ life detection experiments that need amino acids, polyclonal antibodies and other delicate components

Biosphere collisions can be mutually beneficial as well as harmful

Earth using us to reach into space and "it will be magnificent" - Robert Zubrin

What if the decision is to keep Mars, Europa or Enceladus biologically isolated for ever?

We are our biosphere's noosphere - its thinking component

Value of experimental biosphere collisions in large kilometer scale habitats first

Seeding Earth life on exoplanets

Aim for "win win" rather than uneasy compromise between colonization enthusiasts and astrobiologists

Bibliography 


\section{Abstract}

Proposed near surface habitats on Mars include liquid brines, and fresh water below clear ice. A Europa lander could also encounter water. Some are calling for planetary protection measures to be relaxed (Fairén et al, 2017), Others say we have time to study Mars before Earth life is irreversibly introduced (Rummel et al, 2017).. We argue that when our spacecraft may encounter habitable liquid water, we should aim to be even more ambitious. Some commercial components operate at $300{ }^{\circ} \mathrm{C}$. Building on suggestions from a Venus rover study (Sauder et al, 2017), and a Europa cryobot (Wilcox, 2017), we can aim for $100 \%$ heat sterilization during interplanetary flight. In the best case, colliding biospheres could be mutually beneficial, or occupy distinct niches, but in the worst case Earth life has no defences (Lederberg, 1999b) and survives only in enclosed habitats. By examining suggestions for RNA world cells (Szostack, 2016) we argue that the worst case for Mars is extinction of all native life. An astrobiological survey is an essential previous step to inform decisions about whether and how to land humans on Mars. The technology for heavy lift for humans to Mars would permit it to be completed rapidly.

\section{Extended abstract}

With new ideas about the potential habitability of microhabitats in the top few centimeters of the Martian surface, it's now thought that microbes brought on human missions to Mars could lead to irreversible introduction of Earth microbes to the planet. These could encounter native martian life which could also be returned to any habitats for astronauts on Mars and indeed, back to Earth on rockets that shuttle back and forth between Earth and a Mars base.

We investigate what could happen during such a biosphere collision in some detail, including some situations rarely or never discussed in the planetary protection literature, such as misincorporation of similar but non identical amino acids, which could cause motor neurone diseases. The worst case scenario is that the immune systems of Earth life might not recognize martian life, and mystified by it, offer no resistance, a scenario outlined by Joshua Lederberg (Lederberg, 1999a) (Lederberg, 1999b). We suggest a Drake type equation as a way to clarify thought about the probability of such outcomes.

In the forwards direction we look at the possibility of an early form of life on Mars, or in the oceans of Europa or Enceladus, perhaps resembling the RNA only protocells studied at Szostack labs (Szostack, 2016). We present arguments to suggest that if such life were to still exist on Mars today, it might offer no resistance to colonizing Earth microbial life, and this could lead to complete extinction of all native life that uses the alien biochemistry.

We suggest there is no substitute for understanding any native life on Mars before making the decision about whether to send humans to the Mars surface, and what measures are necessary to protect both Mars and Earth. We show that without this knowledge, human quarantine does not protect Earth from an alien exobiology from Mars. 
Some authors have suggested that we relax planetary protection measures for our rovers, in order to study potential habitats on Mars, knowing that this is likely to introduce Earth life to them irreversibly (Fairén et al, 2017), Others suggest we still have time to send adequately sterilized rovers to the planet to learn something about astrobiology on Mars before humans get there (Rummel et al, 2017)?

We propose that we should respond to the challenges by being more ambitious rather than less so in the field of planetary protection. With new advances in technology, $100 \%$ sterile rovers are a feasible near term goal through heat sterilization. Ideas for a complete rover built of such components have been developed as part of the Venus Rover studies and the proposal to use the same approach for planetary protection is suggested in one of these studies (Sauder et al, 2017, section 6.2).

We suggest that this is a matter to explore with some urgency. The project can share some of its goals with the Venus surface rover project.

We suggest ways that humans and robots can work together to unravel the astrobiology of Mars in a way that avoids all possibility of such worst case consequences. Astrobiologists favour an in situ search first, and there are many miniaturized life detection instruments we can send to Mars to look for life.

We suggest that this can be done within the context of a vigorous and inspiring approach to human exploration that continues to respect planetary protection, leading eventually to human bases that have been proposed as far as Callisto and Titan. Callisto, the outermost of the larger moons of Jupiter has abundant resources of ice and organics, lower radiation levels than Mars and the same planetary protection classification as the Moon (Adams et al, 2003) (Kerwick 2012) (McGuire et al, 2003)

Elon Musk's BFR or similar technology, perhaps assisted by ion propulsion for a faster transit to the Jupiter system, could help to make all this a reality. After this, Titan in the Saturn system would be another goal.

These new technologies can also greatly accelerate the pace of astrobiological exploration of Mars. We can send many rovers to Mars, our mobile sense organs in the solar system. Meanwhile, as humans explore the Moon, they can search for the predicted cryopreserved meteorites from early Earth, Mars, Ceres and Venus at the lunar poles (Armstrong et al. 2002).

Once we have the capability, they can direct the rovers on the surface of Mars via telepresence from orbit, with binocular vision and haptic feedback as for the HERRO study (Oleson et al, 2013) and the first part of the Lockheed Martin "Stepping Stones" to Mars (Hopkins et al, 2011) (Kwong et al 2011) and Mars Base Camp (Cichan et al, 2017) studies as far as the human base camp on the moons of Mars exploring the surface via telepresence, They can also explore Phobos for the samples from throughout the history of Mars that are predicted to lie in its regolith as a result of impacts on early Mars. Our astrobiological understanding of Mars should expand rapidly. 
With this approach we may soon be able to make informed decisions about whether to send humans to the Mars surface. If the decision is made to do so, we will know how to do it in a way that preserves the interest of native martian life and is safe for our astronauts, and Earth itself.

The capabilities to identify life in situ, and also to drill, are both necessary to give a reasonable chance of resolving central questions of astrobiology (Paige, 2000), (Bada et al, 2009), (Davila et al, 2010), Mars gets a constant influx of organics from meteorites, interplanetary dust and comets as well as indigenous organics produced by abiotic processes (Westall et al, 2015).

The Mars 2020 caching rover (Perseverance) has no in situ life detection capabilities, and so would miss minute traces of life such as are found in the hyper arid core of the Atacama desert. It is also unable to drill to any depth, and is unlikely to spot the faint traces of ionizing radiation damaged past life if any exists on the surface. Past life may have been present for short periods of time, and it is not certain that any past martian life had photosynthesis, both reducing the potential for build up of organics (Westall et al, 2013).

Perseverance would also miss thin layers of brines only a couple of centimeters below the surface. Instead it's well equipped to identify rocks similar to our martian meteorites and certain carbonaceous chondrites. Such rocks would be its top priority samples to cache. These are likely to be as ambiguous for astrobiology as the martian meteorites we have already (Bada et al, 2009).

Using estimates by the NRC for the time to completion of a sample return facility (NRC, 2009), and an outline of required legal processes by Margaret Race (Race, 1996), we suggest that it would take at least 20 years from when they first start the legal process through to the first launch of a mission to collect the samples. This makes 2040 an ambitious target for returning unsterilized samples cached by Mars 2020, if they start the process today.

For astrobiologists, this Mars sample return double decade flagship mission is of most interest as a technological demo at this stage (Bada et al, 2009). However the decision has been made and it seems certain that Mars 2020 will launch to Mars and cache these samples for return to Earth. How then can we make most use of it for astrobiology?

We propose an alternative approach to a direct sample return to Earth's surface, to avoid the 20 year delays. Our proposal is to return the sample to above GEO, with part of the sample sterilized and sent on to Earth. This could be done within the current laws, with details worked out through COSPAR workshops, similarly to comet and meteorite sample returns. If there is anything of astrobiological interest in the samples, it can be studied telerobotically, above GEO, and only returned to Earth once it is thoroughly understood. At that point we can design any necessary precautions to protect Earth. 
Such a sample return is not likely to advance our knowledge of whether Earth life is safe for Mars or native Mars life is safe for astronauts or Earth. However as a technological demo it can be useful preparation for later when we have identified samples of astrobiological interest on Mars and wish to return them to Earth, or as close as possible, for detailed study.

Our recent history has shown an increasing need to give thought to the effects of our actions on future generations. The first major wake up call was with the American passenger pigeon. Vast flocks dwindled to a single pigeon and then none at all with shocking abruptness (Yeoman, 2014). Using the lessons learnt, we have saved many other species from extinction. We have acted together at a global level to solve many problems that resulted from our technology and are continuing to work together on many more.

As we travel into space we are likely to need to give more thought, rather than less, to consequences of our actions, and to continue to work together as we meet new challenges. We are our biosphere's way to transfer higher life forms into space. We suggest that we are also its noosphere, its intelligence, that it can use to guide future development in space in a responsible way and avoid possible catastrophic consequences. We suggest that continuing planetary protection at the highest standard is of the utmost importance as we explore space. 


\section{Introduction}

Article XI of the Outer Space Treaty (UN, 1966) presents the need to prevent "harmful contamination" of celestial bodies in the forwards direction, and "adverse changes in the environment of the Earth from the introduction of extraterrestrial matter" in the backwards direction.

In the backwards direction, discussions of how to protect Earth often focus on human diseases, and the Apollo 11 astronauts quarantine facility was intended only to protect against diseases for humans. Carl Sagan's example of Legionnaires disease shows that this is a reasonable concern. It shows that a disease of biofilms from another planet, such as Mars, could use the same methods to attack human lungs (Alberts et al 2002), (Abdel-Nour et al 2013).

At the time astrobiologists thought there was a remote possibility of microbial life in habitats below the lunar surface. Sagan calculated that there could be a layer of organics at a depth of tens of meters and that this would also be optimal for temperature (10 $\mathrm{C}$ at 10 meters depth) and moisture, concluding $t$ "neither should the possibility of lunar paleontology be overlooked... Even if the chances of success are small, attempts should be made to detect lunar subsurface autochthon, both living and dead." (Sagan, 1961).

Even before the astronauts entered quarantine, there was an earlier point in the Apollo 11 mission at which any microbes from the Moon could have escaped and contacted the environment of Earth. That was when the door of the capsule was opened for the astronauts to exit onto a raft in the open sea, and dust from inside the module must have got into the open ocean.

Experts consulted by NASA strongly advised against this procedure, but their alternative proposals were overruled. NASA mission planners cited concerns about seasick astronauts and the difficulty of using a crane to hoist the module onto a carrier, and such considerations consistently took priority over planetary protection in these discussions (Meltzer, 2012, page 226).

A microbe that undermined the marine food chain could have been as harmful for us as a disease of humans, or worse.

Luckily, the Moon turned out to be sterile of microbial life (as most already suspected before Apollo). That may not necessarily be the case with Mars however.

The aim of this article is to move beyond the focus on potential diseases of humans to look at the widest context of the potential harm from the clash of biospheres. We will look at this in both directions, forwards and backwards. This then can help us to decide how to prevent the worst effects of biosphere collisions. The term "biosphere collision" comes from the title of Meltzer's book "When Biospheres Collide" (Meltzer, 2012). 
Biosphere interactions in the backwards direction can include infection of life forms other than humans with microbial diseases, as well as bacterial grazing and predation (Martin, 2002) (Velicer et al 2009). They can also involve exotoxins, such as c. botulinum which can contaminate food in anaerobic conditions, and when ingested by humans can lead to the potentially fatal disease of botulism (Warmflash et al 2007). Chyba gave the example of cows killed by algae in Lake Superior. Algae are not natural predators of cows (Chyba, 2002).

There are many other possible effects from a clash of biospheres with different biochemistries. Some of these interactions don't seem to have been explored in planetary protection papers before.

Certain algae blooms produce BMAA. In laboratory experiments this can get misincorporated into human cells, substituting for I-serine, and is a putative cause for the motor neurone disease ALS, or Lou Gherig's disease (Dunlop et al, 2013). There is no benefit to the algae, and no adaptation to humans, no more than there was for the algae in Lake Superior killing cows. In the case of BMAA,

This time BMAA is not a toxin. It gets misincorporated because of its accidental partial resemblance to I-serine. It is rare for an interaction like this to happen on Earth where all cells have the same biochemistry, and a common vocabulary of amino acids. Could this be more commonplace when the colliding biospheres have biochemistries based on a different vocabulary of amino acids? For instance, is there a significant risk of pervasive motor neurone diseases after biosphere collisions?

Proteobacteria in our gut may provide some protection by removing it (Baugh et al, 2017). However there might be no microbes to protect us from misincorporating similar close analogs of our amino acids from an alien biochemistry.

For instance, micro-algae including cyanobacteria produce many secondary metabolites, These include allelopathic compounds - which hinder competition from other microbes, and toxins. The natural function of the toxins are less clear but may be to deter against competition as well, perhaps including micro-invertebrates (Leflaive et al, 2007). Cattle are often killed in large numbers by the hepatotoxins (liver destroying toxins) produced by the blue-green algae (Agriculture Victoria). These also kill dogs that eat algal mats_(Hoff et al, 2007). They can also emit dermatoxins (damage the skin) or neurotoxins (Leflaive et al, 2007).

Other issues could result from secondary metabolites, which are used (amongst other things) for chemical signaling, and could be accidentally toxic. Other issues could result from metabolic intermediaries, protoxins (that become toxic only after metabolic processing) and allergens, such as the ones that cause asthma. Waste products and metabolic intermediaries could also be accidentally toxic or allergenic. 
The chemistry of alien cells may itself be toxic to Earth life. One suggestion is that Martian life might use hydrogen peroxide and perchlorates in its intracellular fluids in place of the chlorides used by Earth life, similarly to the composition of the brines it inhabits

(Schulze-Makuch et al, 2010). This could adversely affect Earth microbes that interact with martian cells or scavenge dead martian life.

We will also discuss life that evolved novel capabilities in the environment of another planet, capabilities that no Earth life has. As an example of a minor but significant nuisance, Curiosity detected perchlorate brines at temperatures below $-70{ }^{\circ} \mathrm{C}$. It detected them in the in sand dunes through humidity of the air above them. (Martin-Torres et al, 2015). Many of the salts on Mars are chaotropic agents that can speed up biochemical reactions at low temperatures (Schulze-Makuch et al, 2010).

Mars also has widely varying temperatures between day and night in the top couple of centimeters. Perhaps life could evolve that is capable of surviving both at $-70{ }^{\circ} \mathrm{C}$ and in warmer conditions. If such life is possible, it would be selected for and may exist on present day Mars. Such life perhaps could also survive on Earth. An example might be black yeasts that may be able to survive on Mars (Zakharova et al, 2014). Could equivalent microbes on Mars use chaotropic agents to grow at much lower temperatures than the $-20{ }^{\circ} \mathrm{C}$ our freezers operate at today?

Some forms of life could have a major impact in the backwards direction. For instance, the thin atmosphere, low light levels (half that of Earth) and frequent dust storms could favour photosynthetic life that is more efficient at fixing carbon dioxide than Earth microbes. What would that do to the marine food chain, if they came to replace the phototrophs in our oceans, but had toxic byproducts or were inedible or toxic to Earth life? And what would the long term effect on our atmosphere be, over millennial timescales, if the new phototrophs from Mars do not produce oxygen?

Then finally, there's the possibility that Earth life's immune systems don't recognize the martian microbes as life, and so present no defences to it. If that happened, martian microbes might have a "field day in light of our own total naivete in dealing with their "aggressins". (Lederberg, 1999a)

The worst case in that final situation could be that few higher lifeforms on Earth are able to evolve fast enough to develop defences against microbial invaders from Mars.

We will argue, following Sagan, that it's important to ensure that such a worst case scenario can't happen, even if the chance of it is small.

Mars seems far more habitable than was assumed a decade ago. The situation has changed so much that in modern planetary protection discussions, it's generally assumed that after a human landing, introduction of Earth microbes to Mars is irreversible, and that we will no longer be able to study Mars in its pristine state. The discussion focuses more on how long this would take, and whether there would be enough time for an astrobiological study of 
Mars before it happens, rather than whether it would happen (Fairén et al, 2017), (Rummel et al, 2017).

We are in a situation where we not only hypothesize the presence of brines on Mars, but we have clear evidence that the brines exist. So the prospects for native life on present day Mars are greater than they seemed to be for the moon at the time of the Apollo mission. The prospects are greater still for life in Europa or Enceladus with their subsurface oceans.

If this is true, and if there is native life in any of these places, then there is a significant risk that this time there really will be a biosphere collision.

In the case of the Moon, no harm was done. We soon found that it seems to be sterile of any life. Already, before Apollo 11 launched, the chance of life there was considered to be very low. The quarantine rules were soon relaxed. The precautions are now thought to not have been adequate in the first place. We may have learned more from the mistakes made in planetary protection for Apollo than from the measures they took to protect Earth (Meltzer. 2012).

However, this doesn't mean that the same lax approach to planetary protection will continue to work in the future.

We might have been lucky with the Moon. We can't rule out the possibility that extraterrestrial civilizations at a similar stage of development to us run into major issues when they send their first space missions to nearby planets or moons. Perhaps it is more typical for them to return life that degrades the environment of their planet through biosphere collision. We may have been lucky to have a nearby large and sterile moon for our first missions into space.

One possibility for Mars, and indeed the Europan and Enceladus oceans, is an early form of life, equivalent to whatever existed on Earth before LUCA (the Last Universal Common Ancestor of all known Earth life). The First Universal Common Ancestor may not be the same as LUCA, indeed probably was not. Also the first ancestor(s) may not be unique. There might have been many early ancestors sharing capabilities via lateral transfer in a community of microbes. This is especially likely if there was an early phase of primarily Lamarckian rather than Darwinian evolution (Woese, 2002) (Brown, 2003) (Jheeta, 2013).

Whatever our first ancestors were, they must have been far simpler than modern life, with its two biopolymers and huge complex enzymes such as the ribosomes. Microbiologists have been unable to find any sign of life as simple as this anywhere on Earth. Could they be extinct? Could it be that such early and primitive life forms had no defences against modern life when it evolved?

At least, whatever they were, we have found no sign of them in any of the habitats studied so far on Earth, although there has been a search for a "shadow biosphere" to try to find them. 
The outcome is impossible to predict, in either direction, when we don't yet know anything about the nature of native martian life (if it exists). We will argue that we should learn the nature of the martian life before embarking knowingly on such a collision.

Although panspermia is possible in theory, it's also possible that the two planets have never shared life. Most life on present day Mars would inhabit either centimeters thick surface layers or deeper caves, neither of which are easily transferred to Earth after a meteorite impact. The martian meteorites are all known to come from meters below the surface, from studies that disentangle the ionizing radiation effects on the meteorites before they were ejected from Mars. Impact modeling of the grazing impacts also shows that they have to come from below the surface. Also such impacts couldn't transfer dormant or active life in the fragile surface habitats in ice, salt or sand dunes. The deep subsurface is likely to be sterile apart, possibly, from rare geothermal hot spots and caves.

In the opposite direction, transfer of life from Earth to Mars is especially hard because of the high gravity, which increases the escape velocity and so the shock of ejection, and the thick atmosphere. This has the result that any meteorite exiting our atmosphere at the Earth escape velocity of $11.2 \mathrm{~km} / \mathrm{sec}$ gets enveloped in a blazing fireball throughout its flight from the ground through to space. The surface layers are ablated. Exposed surfaces that remain after the ablation are sterilized, as well as any cracks and pores in the rock that the plasma of the fireball can enter (Cockell, 2008). Any remaining life deep within the rock would be unlikely to infect the modern cold and arid Mars.

This paper will look at what we can do if this analysis is correct, to explore space in a responsible way. The aim is to find a way to do so that protects our astronauts, and Earth itself. It should also encourage good science, and preserve our heritage for future generations including any interestingly different extraterrestrial life in our solar system. It also needs to be consistent with a vigorous and exciting exploration by human beings and possible human settlement in the solar system.

We will suggest that this is not yet the time to relax planetary protection requirements, with those potential implications that could range from minor nuisances such as spoilt food in fridges and freezers through to major impacts such as the possibly complete extinction of the alien biochemistry of an entire biosphere on Mars, Europa, or Enceladus, or severe degradation of the environment of Earth. Rather than aim for an uneasy compromise where nobody is happy, we can find a "win win" situation where we all aim to be more ambitious rather than less so.

One way to explore the solar system in an ambitious, and yet responsible way is to focus human efforts on the moon to start with, followed by telerobotic exploration of Mars in the context of a vigorous program of human exploration that sets its sights on eventually sending humans to Callisto in the Jupiter system. This has similar planetary protection status to the Moon, has abundant ice, organics from carbonaceous meteorites and other resources and has lower radiation levels than Mars (Adams et al, 2003). 
As later ambition, with faster rockets or good closed system recycling we can also set our sights on Titan in the Saturn system.

Titan is an attractive target for a human base, since it is one of the few places in the solar system where a spacesuit is not needed, just high tech thermal protection (Nott 2009), due to its greater than Earth pressure atmosphere. Thermal protection is far easier to arrange than protection from vacuum conditions.

Titan has abundant wind and hydro power (Hendrix et al, 2017), organics for making plastics, a stable environment and complete protection from ionizing radiation and large meteorites. Titan's planetary protection status needs to be confirmed but forward and backward contamination of any native Titan life seems unlikely at such low temperatures.

The main contamination risk would be of flows of liquid water (cryovolcanism). If liquid water is present, it would need careful study first, as this would provide a possible habitat for Earth life as well as a habitat that could have alien life that our astronauts and Earth need protection from (Wohlforth et al, 2016a) (Wohlforth et al, 2016b).

Once we can build habitats using materials from asteroids, and icy bodies, spinning for artificial gravity, the entire solar system out to Pluto and beyond opens up to human settlement using thin film mirrors to concentrate the sunlight (Johnson et al, 1977)

This paper will suggest that we may have a heritage of martian life in our solar system, left to us by the processes of evolution. We should leave landing humans on the surface of Mars until we have a clear understanding of the effects of our actions may be.

Any life on Mars is as much a part of our common shared heritage as the Mona Lisa or the parthenon, or great wall of China. We need to be able to evaluate not just what we would gain with humans on the surface, but also, what we would lose, if the trillions of microbes that travel with us extinguish native life on Mars.

In parallel with these more ambitious human exploration programs, and benefiting from reduced costs for heavy lift, our priority should be to resume a vigorous program of in situ astrobiological exploration of our solar system.

Mars can be explored robotically, and then telerobotically, with humans in orbit and on its two moons. This would involve both humans and robots, each doing what it does best, in a valued partnership. The astronauts would be involved in the search for life, controlling robots directly through telepresence and haptic feedback whenever there is a need for on the spot decisions using human intelligence. The robots are our collective sense organs on other planets. Torrence V. Johnson, Galileo Chief Scientist, put it like this in the foreword to Meltzer's "Mission to Jupiter" (Meltzer, 2007)

"There is always a tension in the national debate about how much robotic exploration (such as Galileo) we should do versus so-called human exploration (such as Apollo). This misses the point! What we call robotic exploration is in fact human exploration. 
The crews sitting in the control room at Jet Propulsion Laboratory as well as everyone out there who can log on to the Internet can take a look at what's going on. So, in effect, we are all standing on the bridge of Starship Enterprise"

We will also look in detail at the potential for $100 \%$ sterile landers. This is especially desirable if the rovers and instruments come into direct contact with liquid brines, or approach them closely.

This was an impossible goal at the time of Viking, but due to modern developments of high temperature instruments and mechanical components operating at up to $300{ }^{\circ} \mathrm{C}$, then it should be possible to develop rovers that can be made $100 \%$ sterile through heat sterilization, heating the entire spacecraft to $300{ }^{\circ} \mathrm{C}$ during the voyage to Mars. Though this would require more initial work, a similarly vigorous program is already underway for a Venus surface rover capable of operating at much higher temperatures than would be needed for heat sterilization.

With a similar program for planetary protection, sharing some of the same goals and insights, we soon would have $100 \%$ sterile rovers to explore special regions on Mars as well as subsurface oceans of icy moons and dwarf planets. If the target temperature is $300{ }^{\circ} \mathrm{C}$ or lower, most or all components we need are already in use commercially, for applications such as oil wells, lighter aircraft engines, and electric cars, or have been shown to be feasible in Venus lander studies. The main thing needed is to integrate them into a heat sterilizable rover using insights and technology developed for a Venus rover. Some method would need to be found to supply the experiments that need organics that are not heat stable. But if we can achieve all this, which seems within reach, the payoff is huge.

With high bandwidth robotic exploration, with telerobotic human involvement from orbit, we could complete the astrobiological survey in a short period of time. It might take no more than a decade. We could send as many missions as are needed, including dozens of lightweight micro-rovers, cave bots, ballistic penetrators and aerobots in a single mission, without any concern about contaminating the special regions on Mars with Earth life.

We will also propose a method for safe return of samples to above GEO. This would eliminate many legal complexities, technical issues, safety concerns, and design innovations and testing needed for a Mars Receiving Facility in the Earth's environment. We estimate that the time saved by this would make it possible to study unsterilized samples 20 years or more earlier than would be possible if we require a receiving facility to be approved, running and thoroughly tested in the Earth's environment first. This approach also has the potential to completely eliminate any possibility of harmful degradation of the environment of Earth.

In the process we also learn about the Mars conditions and any biology there which may let us avoid expensive mistakes and unforeseen biological hazards if we do decide to send humans there in the future.

Above all, if we have been lucky enough to inherit a solar system with truly extraterrestrial life in it, even if it is only microbial, then this is a way to make sure we leave a similar 
heritage to our children and grandchildren. With the heritage of future generations in mind as well as the heritage of our own generation in future decades, it seems important to consider carefully whether our actions could make other indigenous life forms in our solar system extinct.

Native indigenous life may be more vulnerable than one might have first expected, as a result of the wide range of possible interactions in a biosphere collision. Those potential vulnerabilities are the central topic of this paper.

\section{Planetary protection measures under scrutiny}

Our planetary protection measures are under increasing scrutiny at present, due to the rapid pace of development of private launch capabilities, and the prospect of near future human missions to Mars. Some astrobiologists are calling for a relaxation on planetary protection measures, to study potential habitats on Mars before they are irreversibly contaminated with Earth microbes (Fairén et al, 2017). Others argue that we still have time to study the astrobiology of Mars before irreversible forward contamination occurs, while taking adequate precautions to protect potential habitats from Earth microbes (Rummel et al, 2017). The debate continued in articles in 2018 (Fairén et al, 2018) (Rummel et al, 2018).

Both sides in this contemporary debate are in agreement on the central point. We should treat conditions on and near the Mars surface as potentially habitable to Earth microbes, and we should assume that introduction of Earth life to the planet is likely to be irreversible, at least until we know more.

The proposal of a lander on Europa raises new planetary protection issues. So far we have not sent any spacecraft that could introduce Earth microbes to either Europa or Enceladus. How vulnerable might these bodies of water be to introduced Earth life?

\section{Example of early life on Mars shows potential for complete extinction in forward direction}

Modern DNA based life reigns supreme on Earth. Despite hopes for a "shadow biosphere" (Cleland, 2007), no other form of life has been found.

Wherever we look, in every habitat, however hot, cold, dry, salty, or humid, the only form of biochemistry we find is based on DNA. Moreover, all Earth life uses the same bases, same amino acids, same translation tables, same enzymes, and the same metabolic pathways.

However, this modern DNA life is far too complex to have arisen in one go (Cornish-Bowden, et al, 2017). It could have taken millions of years, or hundreds of millions of years for early life to evolve to this level of complexity. 
Suppose that life on Mars, Europa, or Enceladus has not yet evolved as far as modern Earth life has? Suppose it is slower at metabolizing, slower at reproduction, and has evolved no defenses against modern DNA based life? Perhaps it has no DNA, only RNA (or other form of XNA, at any rate a single biopolymer).

Although we don't know what early life looked like, one possibility is that it resembled the RNA only protocells currently being researched in the Szostack lab (Szostack, 2016). These protocells:

- have no proteins

- replicate as elongated strands that split when agitated,

- have RNA, but with a non uniform backbone to the RNA (which actually helps with the primitive form of replication it uses). No DNA

- use catalysts such as manganese ions to assist replication, and don't yet have any specific enzymes to speed up replication.

The RNA does not yet have a functional role in these cells but they are hopeful that it will in the future, and that the cells will then be able to evolve to greater complexity by themselves.

Earlier, Szostack proposed a hypothetical RNA world cell for Mars, as a possible explanation for nanoscale cell like structures in ALH84001. This was a more evolved cell than his experimental protoecells, but still far simpler than any known Earth microbe. His cell would long predate LUCA, if related to Earth life. He proposed a cell with 1000 ribozymes, with 100 different functions. The debate continues about whether the structures in the martian meteorite ALH84001 are fossil remnants of early life or the results of unusual geochemistry. Whether these particular structures turn out to be life or not, this shows that simple RNA world cells are still seriously considered as a possibility for this meteorite, from 4 billion years ago on Mars.

If Mars could have had primitive cells like this 4 billion years ago, could it still have them today?

Conditions on Mars might have favoured slower evolution than on Earth because for much of its history it has been arid and cold, with surface liquids rare and only marginally habitable, with metabolism probably slow. At times it had more extensive liquid water, but it was oftenn present for short periods of time, or covered in ice discouraging development of photosynthesis. Life may have evolved in isolated oases, and then gone extinct perhaps many times.

"Thus, whatever the scenario for the development of habitability, the relatively short period favorable for the appearance of life-early in the history of Mars (pre-Noachian to Noachian periods)—and the rapid discontinuity of habitable surface conditions (cf. Cockell et al., 2012) imply that, if life appeared on the Red Planet, it probably remained in a very primitive state of evolution, in most cases probably not 
achieving (anaerobic) photosynthesis."

(Westall et al, 2015), see also (Westall et al, 2013)

If it is possible that such life was present back then, could it still be there on Mars now?

These could be colonized by hardy spores imbedded in a grain of dust. But suppose that the subsurface life on Mars never developed these hardy spores, or spore forming life or life with hardy dormant states hasn't reached the surface in recent Martian geological times. In that case, surface environments would be isolated from each other. Even a sand dune would be colonizable only via connected brines beneath the subsurface. Life then could have evolved independently in the uninhabited habitats.

Woese suggested that in early cells from before LUCA (Last Universal Common Ancestor) lateral transfer might have been the dominant way that genes were transferred between cells. He presents this as a data supported conjecture (Woese, 2002),

The LUCA according to this view can't have been a single organism but rather a "loosely knit diverse conglomerate of primitive cells that evolved as a unit", swapping genes amongst each other. It's also possible that cells might have had a shared metabolism, cross feeding each other metabolically too like a modern bacterial consortium. (Woese, 1998) (Doolittle. 2000)

This could help explain why Horizontal Gene Transfer (HGT) is so universal, with genes readily transferred between all species of modern life (Brown, 2003). Lamarckian evolution may have been the dominant form of evolution during the LUCA and pre-LUCA period (Woese, 1998) (Doolittle, 2000) (Jheeta, 2013).

If this theory is correct we need to be especially careful about not introducing genetic material to an environment that may have early cells that may use a similar biology, perhaps a shared ancestor through panspermia, and predate LUCA.

According to one idea, the earliest life, all the way through to the last universal common ancestor (LUCA), might have been simple "modifiable cells" capable of taking up "naked" genetic material which evolved through lateral transfer, by Lamarckian rather than Darwinian evolution (Woese, 2002) (Brown, 2003) (Jheeta, 2013).

Although cells themselves would not yet be in competition with each other, the genomes within them would be. These might evolve some degree of genomic protection, in small vesicles. As described by Koonin:

"Conceivably, such primitive units of evolution could have been represented by small, virus-like replicons that populated abiogenic lipid vesicles or inorganic compartments and were subject to selection for replication efficiency" (Koonin, 2014)

This first stage may have continued for several hundred million years of evolution, through to as late as 3.5 - 3.8 billion years ago (Doolittle, 2000). If so, the transition to Darwinian on 
Earth occurred at roughly the same time as the end of the Noachian period on Mars, the period of most abundant water and seas.

This would place the formation period of the Martian meteorite, ALH84001 at around the time of this transition to Darwinian evolution of the entire cell. Its formation date is estimated at $4.091 \pm 0.030$ billion years ago (Lapen et al, 2010). The cell like structures within it continue to be a matter of debate, with Steven Benner and Paul Davies still supporting an RNA world hypothesis (Benner et al, 2012). If these are indeed cells, some of the structures in ALH84001 are too small for the cell machinery of a modern cell. Could they perhaps be Carl Woese's precursors to LUCA or their equivalent, for Mars?

Woese suggested that in early cells from before LUCA (Last Universal Common Ancestor) lateral transfer might have been the dominant way that genes were transferred between cells. He presents this as a data supported conjecture (Woese, 2002),

The LUCA according to this view can't have been a single organism but rather a "loosely knit diverse conglomerate of primitive cells that evolved as a unit", swapping genes amongst each other. The cells might or might not also have cross-fed each other metabolically too like a modern bacterial consortium. (Woese, 1998) (Doolittle, 2000)

This could help explain why Horizontal Gene Transfer (HGT) is so universal, with genes readily transferred between all species of modern life (Brown, 2003). Lamarckian evolution may have been the dominant form of evolution during the LUCA and pre-LUCA period (Woese, 1998) (Doolittle, 2000) (Jheeta, 2013).

If this theory is correct we need to be especially careful about not introducing genetic material to an environment that may have early cells that may use a similar biology, perhaps a shared ancestor through panspermia, and predate LUCA.

If indeed, evolution was faster in the earlier ocean than on modern Mars, and evolution has slowed down greatly since then, with a half billion years of fast evolution, and then four billion years of slow evolution, perhaps Mars could potentially still have LUCA cells.

This suggests a hypothesis that the modern Mars life could consist of pre-LUCA replicons in vesicles in undifferentiated cells, sharing genetic material with each other readily.

Though primitive, such life could still be adapted to the conditions it inhabits. Mars could have the most perfectly adapted RNA world "modifiable cells" imaginable, with many specialist enzymes and other adaptations to help it to function in its extreme environment. Evolving through massively parallel Lamarckian evolution, easy and fast uptake of capabilities from its neighbours is the priority, and such life might have little by way of defences against modern Earth microbes.

If so, such life may be likely to be easily overcome by modern Earth life, through whatever processes brought an end to this life on Earth, whether by predation or competition with 
cells with a more efficient metabolism, or bacteriophages (if sufficiently related) or whatever caused the transition to Darwinian evolution.

The martian life could also be overcome through lateral transfer of genetic material from Earth life. There are many ways that lateral gene transfer can occur. It can happen through a viral capsule (such as a bacteriophage), a plasmid or small piece of one, from "naked" strands of nucleic acid which could be from dead or long extinct cells, gene transfer agents (GTA's) which are bacteriophage like agents which contain random genomic segments for transduction to another living recipient, and membrane vesicle transfer - small membrane sacs are emanated from one cell and taken up by another (Jheeta, 2013).

Martian life could also co-exist at different stages of evolution. Early life, such as perhaps RNA world life, could exist on Mars in a "shadow biosphere", alongside more evolved life. We haven't found this shadow biosphere here on Earth yet, and maybe it doesn't exist. However since it was a viable hypothesis for Earth (Cleland, 2007), we suggest it is also a viable possibility for Mars. Such a shadow biosphere of earlier life on Mars may be vulnerable to whatever destroyed it on Earth.

There is another possibility too, based on Charles Cockell's work on "Trajectories of martian habitability" (Cockell, 2014). He looks at the idea of life that goes extinct and then evolves again from scratch. He also looks at the possibility of surface habitats that are uninhabited habitats that are isolated from subsurface inhabited habitats.

So, another possible hypothesis for Mars is newly evolved life that's the result of only a few million years of evolution, in habitats isolated from the subsurface. This is a modification of Trajectory 2 in (Cockell, 2014). He envisions a habitat created by a meteorite impact, similarly to the

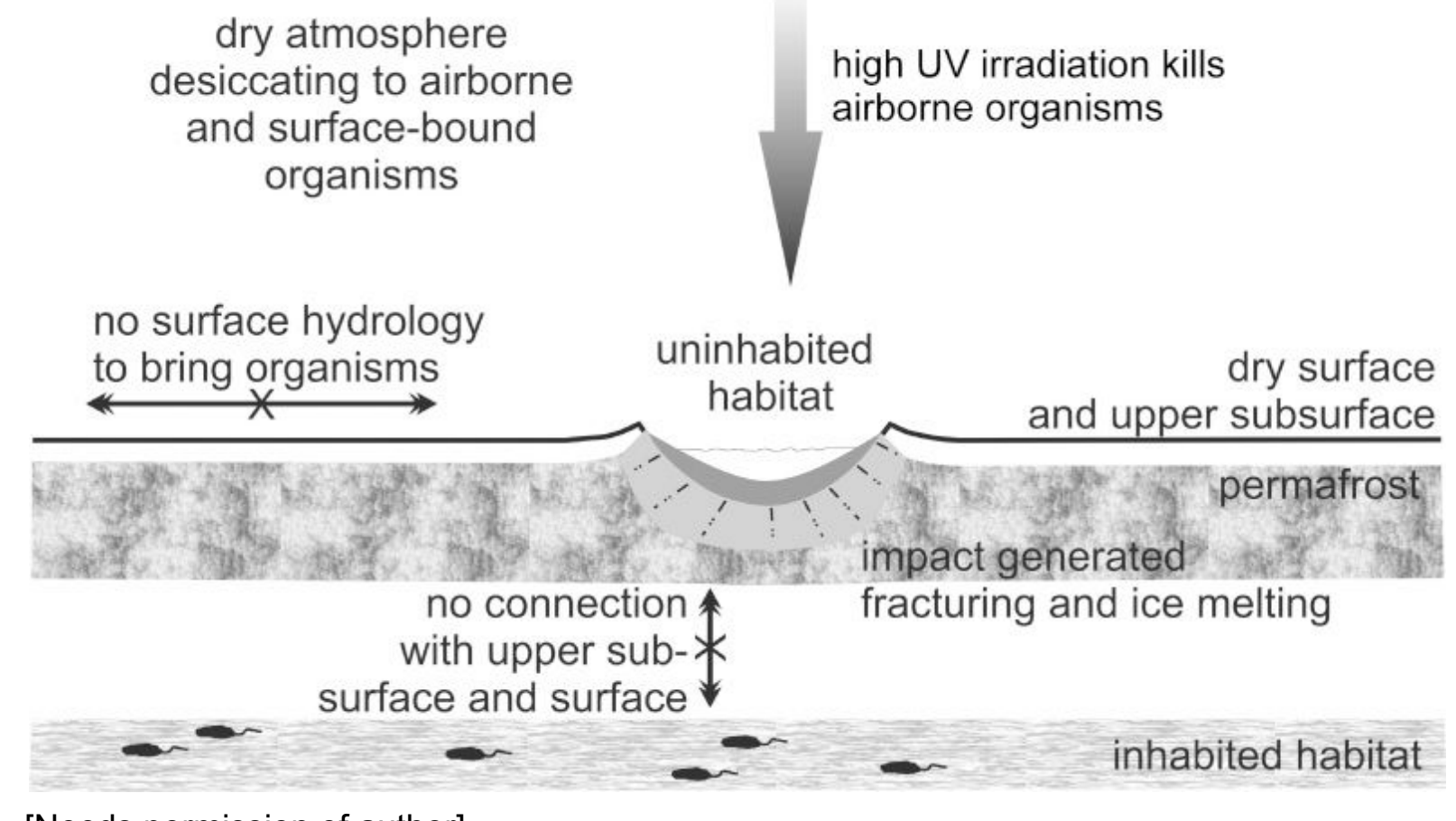

[Needs permission of author] 
This assumes that evolution can proceed at a fast pace in small isolated habitats and that there has been no transfer of life from Earth to Mars in that time. The last opportunity for transfer from Earth was 66 million years ago, but it is also possible that life has never transferred to Mars from Earth.

This seems not an impossible scenario for some of the habitats on Mars, such as the RSLs, or the flow like features in Richardson Crater or even the brines discovered by Curiosity. Life could be localized to them in much the same way that New Zealand was isolated from invasive diatoms from North America (Spaulding, 2010).

This suggests the possibility of another kind of "shadow biosphere" through physical separation. In this case, more evolved life remains deep down in the hydrosphere, kilometers below the surface, perhaps also in caves isolated from the surface similarly to Movile cave in Roumania and Boulby mine. However in some surface habitats, life has evolved again, starting a few tens of millions of years ago, It could then be at a very early stage of evolution.

This reboot of evolution could also happen in hydrothermal vent habitats either after meteorite impacts, or due to volcanic hot spots beneath ice sheets similarly to Blood falls in Antarctica (Aerts et al, 2014, section 2.2.3), or lakes maintained liquid from internal heat like lake Vostok (Aerts et al, 2014, section 2.2.4) such as the possible subglacial lakes near the martian south pole (Orosei et al, 2018, Witze 2018), if the habitats are sufficiently sealed off from any existing life on Mars. It could also happen in caves similar to Movile cave in Roumania and Boulby mine on the North East coast of England (Aerts et al, 2014, section 2.2.3)

Another potential discovery is prebiotic chemistry. Somewhere in our solar system, we might find a potentially habitable planetary surface (e.g. Mars) or an uninhabited habitat on Mars say, or a subsurface ocean in our solar system with no biology; only chemistry. This could tell us much about the early prebiotic stages of evolution, about the prospects of life around other planets in our galaxy, and help us to disentangle the effects of chemistry and of biology on our own planet by comparison with habitats in which only chemical reactions operate.

Prebiotic chemistry has value too. We can't simulate in our laboratories the effect of millions, or billions of years of prebiotic chemistry on another world. Perhaps such habitats could have RNA and autopoetic cells (Stano et all, 2010), but no life. Different habitable regions could differ in the type and complexity of the prebiotic chemistry, again in ways we can never simulate or study once the regions are taken over by Earth life.

To give a few examples, some of these "uninhabited habitats" might have autopoetic cells (Stano et all, 2010) and others Ostwald crystals (Cartwright et al, 2007), formed by crystallization of achiral organic solutions assisted by solution phase racemization (Blackmond, 2010). Or we might find 'naked genes' adsorbed on the surface of mineral 
particles, and perhaps with impermeable membrane caps 'invented' by the genetic system (Leslie, 2004).

Some of these habitats might have one chirality and others the other chirality, and this could help to elucidate the origins of the homochirality of modern life.

If these habitats with prebiotic chemistry are habitable to Earth microbes, how long would they remain in a state suitable for study by astrobiologists and geochemists after infection by even one microbe or dormant spore capable of replicating in them?

Perhaps even an intact microbe or microbial spore is not needed. Infection with fragments of RNA or enzymes from Earth microbes could be enough to give protobionts in these habitats at a late stage in chemical evolution the missing key to become a simple form of replicating life. The resulting life could be interesting in its own right, but this process could erase all traces of the pre-existing protobionts, so that we never get to study them in their original state.

If life is still at an extremely early stage of evolution on Mars, it might be only a matter of time after the first human boots on Mars before introduced terrestrial life reigns supreme in all the habitats on such a world. This process could be hastened by a crash. Elon Musk put it like this:

"I think the first trips to Mars are going to be really, very dangerous. The risk of fatality will be high. There is just no way around it, It would basically be, 'Are you prepared to die?' Then if that's ok, then you are a candidate for going." (Thomet et al, 2016)

A human occupied ship could lose control as it enters the martian atmosphere similarly to the crash of the space shuttle Columbia. Such a crash could scatter debris over thousands of square miles of the martian landscape. For Columbia the debris field was 350 miles long, and about 50 to 100 miles wide (depending on whether you measure to the most distant debris) (Barry et al, 2003).

This debris field could be a source for microbial life from Earth to spread as extremophile spores, and enzymes and genetic material in the dust storms which could then take them almost anywhere on Mars (see UV tolerance by extremophiles and transport of spores in martian dust storms (below)). Such a process couldn't be reversed or stopped.

If we noticed that our microbes were causing extinction of early life on Mars, perhaps enclosures might keep Earth's biology away from parts of Mars, or locations of special interest in the oceans of Europa or Enceladus such as the hydrothermal vents or parts of the undersurface of the ice, and so prevent total extinction.

However, it would be hard to protect local habitats from life spread in the global dust storms on Mars and the girdling oceans of the icy moons. It would be especially hard to construct such enclosures while keeping the interior free of any Earth life. This would require the $100 \%$ 
sterile robots or telerobots, which could be developed but so far do not exist (see Are $100 \%$ sterile rovers and instruments possible in the forward direction?).

The potential biospheres are also so connected that native early life such as this could go extinct before we know what we have lost. Alternatively we could identify the native life before it goes extinct, but find it is hard to get it to replicate in the laboratory or lose our early samples because of contamination by Earth life.

An early martian expedition might discover viable life, but find that the astronauts are not able to keep it alive during the journey back or their samples get contaminated by Earth life within the spaceship. The native life on Mars could be gone before we have learnt how to keep it alive. Typically only $1 \%$ of the species of microbial Earth life is cultivable in the laboratory. Amongst other challenges may be supply of the wrong nutrients, culture media that are too nutrient rich, and microbes that require symbionts in a biofilm to grow (Vartoukian et al, 2010). The culture of a previously unstudied extraterrestrial biology may be a greater challenge than for Earth microbes.

Viable life could also be extremely localized on Mars, for instance, to Recurring Slope Lineae or to the Flow Like Features in Richardson's crater (see Potential Mars surface and near surface habitats)

Depending on how quickly this invasion of Earth life occurs, and how localised the life is on Mars, the invasion could happen so quickly, in a matter of years, that by the time we have the opportunity to study it, or attempt to cultivate it, nothing remains of the native life of Mars, Europa or Enceladus, even in a shadow biosphere. Or if anything remains, it might only survive as clues to its prior existence in the form of capabilities transferred from the native life into Earth microbes, or early life transformed in some way by uptake of biochemicals from modern Earth life.

These possibilities of early life or prebiotic life suggest that the worst case result of accidental introduction of Earth life to an alien biosphere could be total extinction of all life that uses the alien biochemistry, or erasure of all protobionts. In the very worst case we might not be able to cultivate the early lifeforms in a laboratory or replicate the prebiotic chemistry before its inevitable erasure from Mars.

\section{Could evolution on Mars evolve as fast as on Earth, reaching simple animals such as sponges, evolve faster, reaching a genetic complexity we can't yet match?}

Although you can make a strong case for life on Mars to be at an early stage, we suggest that you can also put as good a case in the other direction, for it to be as advanced as Earth life, perhaps even with more complex, with more complex, more evolved genomes than for Earth life. For instance we will suggest it is not impossible that Mars developed its first multicellular life billions of years before Earth did. Genetic complexity needn't mean 
intelligent life, but microbial life, sponges, lichens, molds and so forth with genes that are more complex than any that Earth life has yet developed.

The frequent freezings of the Martian oceans in early Mars, possibly every Martian year when its eccentricity is high, and the ionizing radiation, might have led to populations repeatedly getting reduced to a fraction of the previous population and then rapidly growing again. Boyle et al argued that a similar process led to the development of multicellular life on Earth during its "snowball Earth" glaciations (Boyle et al, 2007).

Their suggestion is that during snowball Earth phases, colonies would often be founded by a single cell from the previous generation, the founder effect, leading to habitats colonized by large numbers of almost identical cells. These cells would be confined to small habitats, and so encounter each other more often increasing the benefits of mutual altruism. The rate of reproduction would also be slow, reducing the benefit to "cheats" that do not contribute to the benefit of the colony as a whole. There would be more importance in mutually beneficial modification of their microhabitat through production of chemicals that are costly for individual cells to produce. They suggest that differentiation of cells, the first steps towards multicellularity, would be especially useful in harsh conditions.

Although they do not apply their theory to Mars, these are conditions that applied to early Mars and to some extent also today, with times when streams of flowing water are possible on the Mars surface, so if these conditions did favour multicellularity on Earth, perhaps they favoured it also on Mars.

Another possibility is that oxygen triggered the explosion of multicellular life. The last common ancestor of the eukaryotes may have lived between 1.855 and 1.677 billion years ago. That's at a time when the oceans were only moderately oxygenated. Most of the varieties (clades) of eukaryotes diverged before 1 billion years ago, probably before 1.2 billion years ago. But the huge diversity we have today within those clades only started 800 million years ago when the oceans started to change to their modern chemical state (Parfrey et al, 2011). Curiosity's Chemcam instrument found manganese oxides which suggest that at the time of Gale crater lake, three billion years ago (NASA video, 2017), the water was oxygen rich (Lanza et al, 2014).

Present day Mars may also have conditions for oxygen rich brines anywhere on the surface, by taking up oxygen from the atmosphere, a process that happens most easily in cold conditions. Extremely cold brines in polar regions could reach oxygen saturation levels similar to those needed for primitive sponges (Stamenković et al, 2018) (Stamenković, 2019. The south pole subglacial lake (Orosei et al, 2018, Witze 2018), if they exist, may provide habitats for multicellular life. These habitats may also be oxygen rich, through radiolysis of the ice, favouring animal life (Stamenković, 2019, interview section on subglacial lakes), (Stamenković et al, 2018).

Mars also has times of volcanic activity leading to hydrothermal systems where ice meets with lava. These lead to lakes that last for thousands of years, as happened 210 million years ago on one of the flanks of Arsia Mons, two lakes with around 40 cubic kilometers of 
water each, and a third one of 20 cubic kilometers of water, liquid for hundreds, or even thousands of years (Scanlon et al, 2014), our infrared mappers can only directly measure the top few millimeters of the surface, and there could be present day hydrothermal systems at depths of up to tens or hundreds of meters below the surface, where biological activity may still survive (Nisbet et al, 2007, page 108ff).

So, perhaps the case can be argued both ways, that the harsh conditions could have slowed down evolution, or that the ionizing radiation and the frequent "snowball Mars" phases, combined with the oxygen rich atmosphere, could have triggered a more rapid evolution on Mars, and possibly even complex multicellular life billions of years before it became common on Earth.

If Mars had multicellular life early on, perhaps that multicellular life is still there, as a relic biosphere. Stamenković et al, 2018 research suggests the possibility of enough oxygen for simple animal life such as sponges exploiting the oxygen in extremely cold oxygenated salty brines when the axial tilt of Mars is less than 45 degrees.

According to their modeling, the tilt is close to optimal for oxygen concentrations now, and for the last 5 million years and at least 20 million years into the future. In polar regions of modern Mars some of the coldest brines supercooled to -123 to $-133^{\circ} \mathrm{C}$. can potentially have as much oxygen as surface water of the Earth's oceans, $0.2 \mathrm{~mol} \mathrm{~m}-3$, or about $6 \mathrm{mg} /$ litre, enough even for spawning migratory fish. The brines can be supercooled that low even when mixed with simulated Martian regolith, and there is no nucleation, the supercooled brine instead transitions to a glassy state.

These low temperatures for the most oxygen rich brines, -123 to $-133^{\circ} \mathrm{C}$ are far too cold for Earth animals, but in those conditions, perhaps exotic biochemistry such as life using perchlorates internally as part of the metabolism, even hydrogen peroxide, may be possible (Schulze-Makuch et al, 2010). With a $\mathrm{H}_{2} \mathrm{O}-\mathrm{H}_{2} \mathrm{O}_{2}$ intercellular fluid, metabolic activity could continue down to $-56^{\circ} \mathrm{C}(217 \mathrm{~K})$ in an eutectic mixture that minimizes the temperature for activity (Houtkooper et al, 2006). If instead they used perchlorates internally, the limit might be reduced further, to $-70^{\circ} \mathrm{C}$ (Houtkooper et al, 2010)

Alternatively the oxygen in these cold brines could serve as a nutrient resource that deeper warmer water may encounter (Stamenković, 2019)

One possibility is that life on Mars and Earth has a common origin, seeded from each other. Both could also be seeded from other stars in the birth nebula of our solar system which could exchange life readily when the stars were closer together (Valtonen et al, 2008) (Belbruno et al, 2012). Life in our sun's birth cluster could also originate in a star older than our sun, spread from cluster to cluster by life bearing stars (Adams et al, 2005)

Sharov found that if you graph the complexity of non redundant nucleotides against the time of origin of an organism, then the log of the complexity increased at a near constant rate of 0.89 every billion years (Sharov, 2006). 
Projecting back if evolution of genetic complexity continued at the same rate since the origins of life, Sharov finds that, Earth life originated around ten billion years ago. If so, life on Earth could be billions of years older than our solar system. (Sharov, 2006)

If the same constant rate of increase of the log of the complexity of the non redundant genomes applies to Mars, the life there could be at an equivalent level of evolution to Earth life. However, if the constant is planet dependent, it could exist at a less advanced level (as discussed in the previous section) or a more advanced level, with genome sequences more complex than we have on Earth at present.

\section{Potential Mars surface and near surface habitats}

It's generally accepted that the Europan and Encaladus oceans have a reasonable chance of being habitable for Earth life.

However, in the case of Mars this is not so clear and there is a wide variety of views on the topic. This is of significance for the question of whether native Mars life could be vulnerable to introduced Earth microbes. If there is surface life on Mars, it is likely to be cryptic, hiding from the UV, in partial shade or a few millimeters or centimeters below the surface. It is also likely to be slowly metabolizing, possibly with individual microbial lifetimes of months through to millennia.

The impact on the atmosphere may well be minimal making detection by monitoring the atmosphere difficult or impossible. Regions of Mars with potential for habitable liquid water are called "Special Regions" in planetary protection discussions, and refer to regions where strict planetary protection is required (Rettberg et al, 2016).

It is generally accepted now that liquid brines are present in the Martian subsurface, with the most definitive evidence perhaps the humidity measurements from Curiosity indirectly proving the presence of a thin layer of cold brines below the dunes it drives over in Gale crater (Martin-Torres et al, 2015). The differences in views are over whether any of these brines are habitable to Earth microbes.

In the case of the brines in Gale crater the answer was that they do not seem to be habitable to Earth life. At times in the daily cycle they are warm enough but have too little water activity and at other times they have enough water activity but at $-70{ }^{\circ} \mathrm{C}$ are far too cold for even the most cold tolerant Earth microbes to flourish (Martin-Torres et al, 2015). However they may be habitable to martian life based on a different biochemistry. Also perhaps they could be inhabited by Earth life in biofilms. This is an insight by Nilton Renno as interviewed by Michigan news soon after the discovery (Fernanda Pires, 2015).

We will cover this in more detail in the section on Unanswered questions about Mars special regions (below)

There are many other proposed habitats now, and some may be more habitable than the Curiosity brines. 
The Phoenix lander's isotopic measurements of the atmosphere in 2008 showed clear evidence of geologically recent and extensive exchange of oxygen between the carbon dioxide in the atmosphere and liquid water on the surface (Niles et al, 2010). Phoenix also observed what seem to be droplets of liquid forming on its legs, merging, and then falling off (Shiga,2009). These two results were what first caused many to re-evaluate their views of the potential habitability of the Mars surface. The top few centimeters particularly may be habitable to Earth microbes as well as to native life.

Since then, Nilton Renno et al have shown that whenever there is calcium perchlorate salt lying on ice, in a chamber simulating the Phoenix lander conditions, then droplets of liquid brines can form within tens of minutes, forming a "swimming pool for a bacteria" as he memorably put it, when summarizing his team's work on this topic (Fischer et al, 2014).

The Recurring Slope Lineae (RSL's) remain a leading candidate for brines that could be habitable, although there is considerable debate in the literature about the amount of brines present and whether they may be habitable. Since they may be the result of aqueous processes, they are characterized in planetary discussions as "an Uncertain Region that is to be treated as a Special Region until proven" (Rettberg et al, 2016).

These dark features extend down sun facing slopes in spring when local temperatures increase above $0{ }^{\circ} \mathrm{C}$, broaden during the summer, and fade away in autumn (McEwen, 2011). The extension and broadening is associated with increased detection of hydrated salts which suggests the presence of thin layers of liquid brines, flowing below the surface of the slopes (Olga et al, 2015).

If caused by brines, the features themselves are not thought to be dust darkened by surface brines, but an indirect effect of the presence of shallow subsurface brines. Later observations of thermal responses to the soil limit the amount of unbound water that may be present in the region of the RSLs to 30 grams of water per kilogram of soil (Edwards et al, 2016). A study of RSLs in Eos Chasma shows that the features are consistent with dust cascades, since they terminate at slopes matching the stopping angle for granular flows of cohesionless dust, and they also ruled out formation of substantial quantities of crust-forming evaporitic salt deposits, though the hydrated salts and seasonal nature continue to suggest some role for water in their formation (Dundas et al, 2017).

Difficulties with the dust explanation include the rapid fading away of the streaks at the end of the season, instead of the more usual fade over decades for dust cascades, and a lack so far of any explanation of how the dust is resupplied year after year. Resupply also remains a major question for the models involving substantial amounts of liquid brines (Stillman quoted in David, 2017). A study of RSLs in the Valles Marineres finds that they seem to traverse bedrock rather than the regolith usual for other RSLs, and that if water is involved in their formation, substantial amounts must be needed to sustain lengthening throughout the season (Stillman quoted in David, 2017). 
Stillman in 2018, suggests some of these features may be caused by dry granular flow, and others by a wet-dominated mechanism (Stillman, E., 2018).

\section{Potential for fresh liquid water in polar regions through the solid state greenhouse effect - of special planetary protection relevance}

This hasn't had much attention in the planetary protection literature, but is potentially one of the most habitable environments for Earth life on the planet, since it gives a method for fresh liquid water to form, within centimeters of the surface. These habitats should exist if there are conditions of optical transparency and thermal opaqueness in the Martian ice and snow to conditions that prevail in Antarctic ice and snow. The ice should melt due to the solid state greenhouse effect.

If these habitats do exist, and from the Antarctic analogues their presence seems likely, then this is a near certainty for an environment on Mars that would be hospitable to Earth life.

This method for formation of liquid water close to the surface is due to Mohmann. His models of the solid state greenhouse effect for clear ice on Mars suggest one of the most striking possibilities, that the solid state greenhouse effect could lead to widespread formation of fresh water just below the surface of the ice. Liquid water could form at a depth of around $6.3 \mathrm{~cm}$ wherever there is optically clear ice on Mars in snow / ice packs, just as it does in Antarctica. In summer, it could form layers from centimeters to tens of centimeters in thickness (Möhlmann 2009) (Möhlmann 2010a).

In another paper he writes_(Möhlmann 2010b)

"This liquid water can form in sufficient amounts to be relevant for macroscopic physical (rheology, erosion), for chemical, and eventually also for biological processes."

His models seem clear enough. The only unknown here is whether Mars does have optically clear ice like this, which is common on Earth in cold conditions like this in Antarctica. The air temperature makes little difference to formation of fresh liquid water in the depths of the clear ice, because the ice is a good insulator, as is the thin atmosphere above it.

Although this could occur anywhere in the polar regions, we have one particular place to look for it. This is one of the two leading candidates for explaining the flow-like features in Richardson's crater in the Southern polar region, After the Martian dry ice geysers erupt in early spring then dark dune spots form and then from these dark flow-like features flow down the slopes from spring through to autumn, meters a day at times The other main candidate for an explanation is Undercooled Liquid Interfacial (ULI) water (Martínez et al, 2013). 
There are several other flow-like features which have some similarities in appearance but are not identical in behaviour or the conditions in which they form. Similar looking features in Russell's crater are probably due to dust flows because they are able to flow up and over small elevations on their way down the slopes, which liquid would not do (Gardin et al, 2010) (these are not the better known linear grooves in this crater which are due to dry ice blocks rolling down the slope but another small scale feature that happens at the head of the slopes). The ones in the northern polar regions flow in much colder conditions and are either due to dust again, or are very cold brines too cold to be habitable (Pommerol, 2013).

However these particular flow-like features in Richardson's crater may be due to fresh water forming beneath clear ice and then mixing with brines and flowing down the slopes or due to Undercooled Liquid Interfacial (ULI) water. In either case the water starts fresh at the source and mixes with brines as it flows out from under the ice and down the slope (Martínez et al, 2013).

There is some debate here in the literature. Some authors while acknowledging the different formation conditions in Richardson crater think that the same model may describe them all, in a way not yet fully understood. If that is the case, the most likely explanation is dust flows as that's the only real possibility for Russell's crater due to the dark features flowing up and over small elevations on the way down the slope (Pommerol, 2013).

If the flow-like features in Richardson crater are not due to fresh water below clear ice, this doesn't change the conclusion that Mohmann's model holds valid for the Martian conditions if there is any ice there with sufficient optical transparency, similar to ice that forms frequently in Antarctica. It would then be a case of looking elsewhere to see if the conditions are present.

If there is clear ice on Mars anywhere at comparable latitudes in the polar region, exposed to the sun in summer, the conclusion of the model is clear, that there should be layers of fresh water form, cms thick, over a period of several days, at a depth of $10-20 \mathrm{cms}$.

This process would be accelerated by the presence of layers of rock at the correct depth. Liquid water can also form through a similar process around meteorites and grains of dust in the snow or ice warmed by the sunlight, with the heat trapped through the solid state greenhouse effect, another process that frequently happens in Antarctica.

This again is based on processes that happen in the Antarctic ice. Dust grains in the ice often produce tiny melt ponds around them in the heat of the summer sunshine. The dust grains absorb the heat (preferentially over the ice), and so heat up and melt the surrounding ice. Then this heat gets trapped because of the insulating effect of the solid state greenhouse effect, because ice traps heat radiation, so forming tiny melt ponds of a few millimeters thickness or more.

Losiak, et al, modeled tiny micron scale dust grains of basalt (2-2 microns in diameter) exposed to full sunlight on the surface of the ice on the warmest days in summer, on the Northern polar ice cap. They found that these tiny dust grains were large enough to provide 
for five hours of melting which could melt six millimeters of ice below the grain. They say that with pressures close to the triple point, on windless days, you should get a significant amount of melting. They speculate that this might possibly explain deposits of gypsum in the polar regions.similarly to processes that happen in Antarctica (Losiak et al, 2014).

This model is based on a previous paper on Antarctic meteorites. The meteorites there are often found associated with gypsum and other evaporates - minerals that can only form in the presence of liquid water and must have formed after they fell in Antarctica. Sometimes the researchers find capillary water, or thin films of water, and sometimes they find evidence of a rather large meltwater pond which formed around the meteorite, or find the meteorites in depressions filled with refrozen ice (Losiak et al, 2011)

This could explain another puzzle. Particles of gypsum have been detected, first in the Olympia Undae dune fields that circle the northern polar ice cap of Mars _Fishbaugh et al, 2007). Later on, they were detected in all areas where hydrated minerals have been detected, including sedimentary veneers over the North polar cap, dune fields within the polar ice cap, and the entire Circumpolar Dune Field. There's strong evidence that the gypsum originates from the interior of the ice cap (Massé et al, 2012). Gypsum is a soft mineral that must have been formed close to where it has been discovered (or it would get eroded away by the winds) and as an evaporite mineral, it needs liquid water to form.

Opportunity later found veins of gypsum in the equatorial regions, in 2011, a clear sign of flowing water on ancient Mars. But these polar deposits are more of a mystery because they are found in the dust dunes on Mars, so must be produced locally, but where?.

Möhlmann did a similar calculation. This time he was looking at the possibility of liquid water forming inside snow on Mars. The snow would be exposed to the vacuum, but as the ice melted it would plug all the pores in the snow and eventually form a solid crust of ice on the snow, and so protect it from further evaporation. It would trap the heat as well and so encourage melting. This could happen anywhere between a few centimeters depth down to ten meters below the surface

\section{Potential deep subsurface habitats communicating with the surface including ice fumaroles and hydrothermal systems on Mars}

This is another habitat of special interest for planetary protection discussions. Mars is geologically active, with small scale volcanic features associated with some of the volcanoes on Mars including eruptions on Olympus Mons as recently as two million years ago (Neukum et al, 2014). There's also evidence of ice near its summit, perhaps even present today, covered by dust. So as recently as a few million years ago, Olympus Mons had ice and hot spots in close proximity, which strongly suggests it had liquid water as well, and so, potentially habitable (Cousins et al, 2011). 
Mars might have present day volcanism, releasing gases to the atmosphere. NOMAD on ExoMars' trace gas orbiter will be looking for geological as well as biologically relevant molecules in the atmosphere. It's targeting carbon, oxygen, sulfur and nitrogen atoms and carbon-hydrogen bonds (Khayat et al, 2014).

Hoffman et al. have suggested that ice towers could mask the heat signature of fumaroles on Mars (Hoffman et al, 2003) in a process similar to the ice fumaroles of Mount Erebus (Tebo et al, 2015). If these environments do occur on Mars, they would provide a warm environment, high water vapor saturation, and some UV shielding. The ones we have on Earth don't have significant amounts of liquid water. However, as they have close to $100 \%$ humidity inside, that doesn't matter. They sustain microbial communities of oligotrophs, i.e. micro-organisms that survive in environments that are very poor in nutrients. The same could be true of Mars. They survive using $\mathrm{CO}_{2}$ fixation and some may use $\mathrm{CO}$ oxidization for their metabolism. The main types of microbe found there are Chloroflexi and Acidobacteria (Hoffman et al, 2003) (Tebo et al, 2015).

They would not be limited to polar regions because the fumaroles themselves would bring large quantities of water vapour to the surface to keep replenishing the ice towers as they sublime away in the thing Mars atmosphere. They might be quite easy to spot as white circles or ovals, probably in permanently shadowed regions. He investigated some anomalies in Hellas Basin as potential ice fumaroles (Hoffman et al, 2003). If Mars is currently geologically active, then in such cold conditions, it may well have ice fumaroles around its vents, and if so they would be only a few degrees higher in temperature than the surrounding landscape and hard to spot from orbit (Hoffman et al, 2014).

He suggests searching for them on Mars from orbit. He located potential temperature anomalies in Hellas basin that seemed promising, at diameter 10 - $30 \mathrm{cms}$ and clustered around a potential fracture, but he was unable to find anything in the visual images corresponding to the potential thermal hot spots (Hoffman et al, 2003). They wouldn't need to be in polar regions because the fumaroles themselves would bring large quantities of water vapour to the surface to keep replenishing the ice towers as they sublime away in the thing Mars atmosphere. They might be quite easy to spot as white circles or ovals, probably in permanently shadowed regions, and they would be slightly warmer than their surroundings.

So, could there be ways that volcanic processes on present day Mars produce habitats by interacting with ice, such as the ice fumaroles? Though we haven't found ice fumaroles on Mars yet, we have found recently formed rootless cones, which are the results of explosive contact of lava with water or ice. This shows that ice (or water) and lava were in close proximity as recently as around ten million years ago (Martel, 2001).

Any interactions today are presumably much less dynamic because Mars is in a relatively dormant phase, but there could be local hydrothermal systems at depths of up to tens or hundreds of meters below the surface, where biological activity may still survive (Nisbet et al, 2007). 
The methane spikes detected by Curiosity, if confirmed, are not likely to be due to fumaroles, or magmatic degassing, as this process doesn't produce much by way of methane on Earth.

They could be due to various surface and near surface processes, both biological and abiotic (Hu et al, 2016) including gas released in the remnants of ancient mud volcanoes and gas rich springs (Oehler et al, 2017), and another hypothesis is that they could be cometary in origin from carbonaceous material falling during meteor showers (Fries et al, 2016). Rettberg et al have suggested that any sources of methane spikes, once identified, should be treated as special regions until proven otherwise (Rettberg et al, 2016)

\section{Lichens, cyanobacteria and black yeasts surviving in modern Mars surface conditions (similar to Gale crater) and other examples of Earth life that could potentially survive on Mars}

Finally, and most striking of all, there's evidence from Mars simulation chamber work, that life on Mars may not need liquid water at all. The hyperarid core of the Atacama desert, and high mountains in Antarctica have life that is able to survive without liquid water at all, using the humidity of the air. Martian life similarly may be able to survive using the humidity of the air due to large day / night fluctuations in relative humidity.

Experiments by DLR on survival of lichens in both Mars simulation experiments show that one lichen, Pleopsidium chlorophanum, could survive and photosynthesize in Mars surface conditions in partial shade_(de Vera et al, 2014).

When exposed to simulated full sunlight the fungal component died and it's not clear if the algal component was active.

However, in partial shade, in a 34 day experiment the photosynthetic activity increased for the duration of the experiment showing that the lichen was adapting to martian conditions. Photosynthetic activity was highest at the start and end of each simulated martian day, at the times when the relative humidity would be highest. The fungal component was also viable and continued growth. The fungus as an aerobe required oxygen from the cyanobacteria to survive.

By the end of the 34 day experiment, it reached $55 \%$ of pre-experiment levels of photosynthetic activity - actually $17 \%$ higher than for Antarctic conditions. Relative humidity varied from 0.1 to 75 percent and surface temperature from $21^{\circ} \mathrm{C}$ to $-50^{\circ} \mathrm{C}$ in a diurnal cycle (de Vera et al, 2014).

The lichen was grown in Sulfatic Mars Regolith Simulant, which is ice free, and resembles the composition of martian meteorites, crushed to simulate the martian regolith, There was no other source of liquid water apart from the atmosphere, at pressures of $700-800 \mathrm{~Pa}$, similar to those in Gale crater. In these conditions cryobrines may have formed in the Mars simulant (de Vera et al, 2014) 
Pleopsidium chlorophanum lives at up to 2000 meters in Antarctica. It is able to cope with high UV, low temperatures and dryness. It is mainly found in cracks, where just a small amount of scattered light reaches it. This is probably adaptive behaviour to protect it from UV light and desiccation since the same species spreads over the surface of rocks in warmer conditions. It remains metabolically active in temperatures down to $-20 \mathrm{C}$, and can absorb small amounts of liquid water in an environment with ice and snow.

Lichens adapted to these conditions have protection from UV light due to special pigments only found in lichens. This gives them enough protection to tolerate the light levels in conditions of partial shade in the simulation chambers and make use of the light to photosynthesize. There are many such pigments used by lichens and cyanobacteria, including the pigments parietin, usnic acid and scytonemin, and antioxidants such as b-carotene. Indeed, UV protection pigments have been suggested as potential biomarkers for Mars (Wynn-Williams et al, 2002)

Other experiments showed that "Two species of microcolonial fungi - Cryomyces antarcticus and Knufia perforans - and a species of black yeasts-Exophiala jeanselmei" could metabolize in Mars conditions too, without any source of water except the humidity in the air, in the close to anaerobic conditions. They recovered the initial metabolic state within seven days and shows no sign of stress reactions. (Zakharova et al, 2014)

These experiments show that the Mars surface conditions are not a barrier to life. As the experimenters suggest in their conclusion, if Earth life can survive in these conditions, Mars life may have adapted to them too, and it is no longer necessary to suppose that native Mars life has to be buried deep below the surface or have endolithic habits protected from surface conditions by rock. (de Vera et al, 2014)

Life relying on deliquescing salt has also been found 2 or more meters below the surface of the Atacama desert. These microbes use mixtures of halite and perchlorates for deliquescence, and they use sulfates, perchlorates $\left(\mathrm{ClO}_{4}\right)$, and nitrates, together with organic acids such as acetate, or formate, as sources of energy. They are quite Mars like conditions really. See A Microbial Oasis in the Hypersaline Atacama Subsurface Discovered by a Life Detector Chip: Implications for the Search for Life on Mars

\section{Microhabitats - such as micropores in salt pillars and ground hugging water vapour as morning frosts evaporate}

The habitability of the Martian surface, with water vapour alone, could also be enhanced by spontaneous capillary condensation in micropores in salt deposits, a process that occurs in the Atacama desert and permits diverse communities of cyanobacteria together with heterotrophic micro-organisms to thrive when there is a virtual absence of cyanobacteria in the surrounding soils (Vítek et al, 2010). 
This occurs at relative humidities far lower than the deliquescence point for $\mathrm{NaCl}$ of $75 \%$. In year round studies of the pillars, they found that the external atmosphere reached a minimum relative humidity of $2.90 \%$, maximum $74.2 \%$ and average of $34.75 \%$ (see table 2 of their paper). So the external atmosphere never quite reached the $75 \%$ level where salt deliquesces naturally, but inside the salt pillars it was able to capture the water in these micropores, easily, and retain it as well. The values for the interior of the salt pillars were: minimum $2.20 \%$, maximum $86.1 \%$, average $54.74 \%$ (Wierzchos et al, 2012)

The authors of those papers suggested this as a possible past habitat for Mars. Cassie Conley and separately, Paul Davies have suggested that these micropores in salt pillars are potential habitats on present day Mars (Conley, 2016) (Davies, 2014)

Gilbert Levin and his son Ron Levin have come up with a way that thin films of fresh liquid water might form on the surface briefly in the morning in equatorial regions of Mars. It's based on findings that the atmosphere up to a meter above ground level remains cold, as it was at night, colder than the surface, and stays too cold to hold the water vapour, as the surface ice melts. This could trap a layer of higher humidity above the warming surface. They suggest that this might lead to thin films of water that form in the early morning, just briefly before the air warms up and the water evaporates into the atmosphere. Chris McKay, commenting on this scenario, agrees that it could form a very short lived layer of liquid, though it may not last for long (Abe 2001).

If this is not sufficient to create habitable films on the surface by itself, perhaps it could enhance the habitablity of the micropores in salt pillars?

\section{Unanswered questions about Mars special regions}

We've seen that Earth life may be able to survive on Mars using just the night time humidity. The humidity could be increased in micropores and as a result of ground hugging moisture when the morning frosts evaporate. There are many other ways the habitability of the Mras surface can be enhanced. These might perhaps extend the range of what counts as special regions on Mars.

Rettberg et al give a list of unanswered questions (Rettberg et al, 2016), and we need to bear these in mind when asking if martian brines are habitable to Earth life.

Two of them are covered already (I paraphrase from section 3.1)

- Is water vapour alone sufficient for replication? (as in the DLR experiments)

- Do conditions in microenvironments on Mars mirror macroenvironments or can they sometimes be more suitable for life?

(As with the micropores and ground hugging water vapour)

- Can a single species microbe from Earth replicate in habitats on Mars?

There are several more questions to look at. 
- Can organisms replicate there if the temperature and water activity separately reach levels suitable for life, but not both at once, for instance by storing water when it is cold and then using it to replicate as the brines warm up?

As we saw, the brines discovered indirectly by Curiosity in Gale crater seem at first to be outside the range of habitability for Earth life. At times in the daily cycle they are warm enough but have too little water activity and at other times they have enough water activity but at $-70{ }^{\circ} \mathrm{C}$ are far too cold for even the most cold tolerant Earth microbes to flourish (Martin-Torres et al, 2015).

However, biofilms can alter a microhabitat, and microbes may be able to retain water for use later on when temperatures are higher. Perhaps they could be inhabited by Earth life in biofilms. This is an insight by Nilton Renno as interviewed by Michigan news soon after the discovery (Fernanda Pires, 2015).

Rettberg et al ask this same question, to paraphrase again:

- Can multispecies colonies form biofilms to help them tolerate environments they couldn't individually?

The final question is:

- How strict is the lowest temperature for replication for Earth life? Have the experiments exploring the lower temperature limits of replication been carried out over long enough timescales to study extremely slowly replicating organisms?

The lowest temperature for replication of Earth life is controversial. The limit is usually given as $-20 \mathrm{C}$, however, it is hard to study replication at such low temperatures because of the long generation times. Experiments with yeast show doubling at $-18{ }^{\circ} \mathrm{C}$, with a doubling time of 30 days. One study showed genome replication in permafrost at $-20{ }^{\circ} \mathrm{C}$ which is "highly suggestive of cell division". Another experiment show ammonia oxidation at $-32{ }^{\circ} \mathrm{C}$ sustained for 300 days, the duration of the experiment. Since cell division would be so very slow at those temperatures, then, so far, it's impossible to be sure whether this is just maintenance metabolism, or whether it actually did support very slow cell division (Rummel et al, 2014).

Chaotropic agents can help microbes be active at lower temperatures. Common examples include ethanol, urea, butanol etc. They work by disrupting the hydrogen bonding of water molecules with each other. The Mars surface has many chaotropic agents which could reduce the minimum temperatures for cell division, including $\mathrm{MgCl}_{2}, \mathrm{CaCl}_{2}, \mathrm{FeCl}_{3}, \mathrm{FeCl}_{2}$, $\mathrm{FeCl}, \mathrm{LiCl}$, chlorate, and perchlorate salts_(Rummel et al, 2014).

Typically, these chaotropic agents reduce the lowest temperatures for cell division by $10^{\circ} \mathrm{C}$ or $20^{\circ} \mathrm{C}$ for many microbe species. However the authors couldn't find any experiments testing these agents at the very low temperatures that would be needed to reduce the lowest temperature limit for cell division for life. (Rummel et al, 2014). 
One study did find that chaotropes can increase the ability of microbes to survive freezing to - $80{ }^{\circ} \mathrm{C}$ with no loss of viability, when frozen for 24 hours, compared with $60 \%$ loss of viability without. The test used the microbial propagules of xerophilic fungi (low water activity) (Chin et al, 2010) but didn't test for cell division at low temperatures.

More radically, however, Dirk Schulze Makuch et al suggested that cold brines on Mars could be habitable to native life with a different biochemistry, capable of living in conditions too cold, or with too little by way of water activity for Earth life. For instance they might have perchlorates inside their cells, taking the place of the sodium. potassium and magnesium chloride salts that we have inside our cells. (Schulze-Makuch et al, 2010).

Based on this proposal, perhaps we can add another question to their list. If native martian life can survive in conditions not habitable to Earth life, perhaps perchlorate or hydrogen peroxide based, could native martian biofilms also store water and make conditions more habitable than they would be otherwise?

- Could Earth microbes inhabit biofilms of native martian life, in conditions inhospitable to Earth life on its own?

(suggested extra question)

The situation for water activity for Earth life is more clear cut, and isn't one of their questions. Most microbes can't handle a water activity level below 0.755 . In a 2014 survey paper, the authors concluded that the best current consensus is that the lowest level of water activity needed for cell division is about 0.605 (the water activity level of honey is 6), and that some halophiles (salt living microbes) are able to tolerate such low levels. The first microbe capable of division at such low water activity levels was Xeromyces bisporus, a fungus that spoils prunes, discovered in 1968 (Pitt et al, 1968), and this record has not been beaten since then. They remark on the difference from the situation for temperatures, that there's this sharp cut off for water activity, but much better evidence of microbes able to tolerate temperatures below the usually cited $-20^{\circ} \mathrm{C}$ (Stevenson et al, 2015)

However if you extend the question to the possibility of biofilms that are not necessarily based on the Earth biochemistry, yet perhaps habitable to Earth life,

- Would a biofilm based on a different biochemistry have the same limitation on water activity, and if so, could Earth life inhabit such a biofilm?

\section{Modern Mars habitability reassessment}

With Mars, much has changed in the last decade since 2008. Though some astrobiologists remain skeptical, some astrobiologists, such as Nilton Renno, and the astrobiologists at DLR, amongst others, are optimistic about the prospect of finding present day native life right on the surface, or within a couple of centimeters of the surface. 
Many of the thin layers of brines on Mars may have too little by way of water activity, or be too cold for Earth microbes. However others are potentially more habitable, including the potential even for fresh liquid water due to the solid state greenhouse effect in polar regions.

A polyextremophile ionization resistant photosynthetic prime producer such as Chroococidiopsis could survive anywhere on Mars that has liquid water, sunlight, some protection from UV (thin layer of dust or even partial shade, since it has pigments that protect against UV), basalt for the trace elements, and a source for nitrogen.

The requirement for liquid water may perhaps be dropped as well as a result of the DLR experiments, as we saw in the previous section, as it could use the night time humidity as the surface warms up in the early morning - and it could also use liquid water that would form in minute quantities in micropores in salt pillars.

There are many redox gradients that life can use as well. For instance in the sand dunes that Curiosity studied, the surface layers are superoxygenated but lower layers less so leading to a redox gradient and slowly moving sand dunes will bring the reducing layers to the surface (Fisk et al, 2013).

The Mars surface conditions, which seemed so hostile a decade ago, seem less so now as the result of discovery that many extremophiles that can tolerate them more easily than expected.

\section{Radioresistant extremophiles like Chroococcidiopsis}

The ionizing radiation is still thought to be sterilizing of dormant life over hundreds of thousands of years, but many ionization resistant microbes can repair both single and double breaks in DNA within hours. Meanwhile, Curiosity's measurements showed that levels of ionizing radiation on the surface of Mars averaged out through the year are similar to the interior of the ISS.

Experiments by Verseux et al show that some desert strains of Chroococcidiopsis can withstand levels of ionizing radiation several orders of magnitude higher than the martian surface. In one experiment, chroococcidiopsis could survive thousands of grays of radiation in the form of any of gamma rays, helium, iron or silicon nuclei, with no detectable loss of viability. By comparison, the absorbed dose rate estimated for the surface of Mars ranges from 50 - 150 milligrays a year. This cyanobacteria is one of our best candidates for a photosynthetic, primary producer, polyextremophile able to survive on Mars

Some cells of chroococcidiopsis still survived after more than $10 \mathrm{kGy}$ corresponding to 100,000 years of dormancy in Mars surface conditions, and one strain had survivors after more than $20 \mathrm{kGy}$. (Verseux et al, 2017). 
We have to drill to find any native martian microbes dormant for millions of years, unless they are more radioresistant than chroococcidiopsis. However, this is only for dormant life.

Radioresistant microbes could cope with the conditions on the martian surface, even if in the cold arid conditions they have a slow metabolism and generation time of millennia.

\section{UV tolerance by extremophiles and transport of spores in martian dust storms}

The UV is also better tolerated than expected, with lichens and some microbes able to withstand exposure to simulated Martian UV indefinitely in partial shade in the DLR experiments.

The perchlorates which provide favourable conditions for cold brines on Mars are used by some microbes as oxidants for their metabolism_(Oren et al, 2014).

In an experiment by Wadsworth et al, when brines on Mars are irradiated by UV, this will produce the more toxic chlorites and hypochlorites. However biofilms would provide extra protection. Also the microbe they studied, Bacillus subtilis, was not an extremophile. Also the habitats on Mars are often wholly or partially shaded from UV light (Wadsworth et al, 2017).

Also, this biocidal effect might not significantly impact on spores spread in the dust storms. Spores are protected by a coat and are far more resistant to oxidizing agents, bactericidal agents chlorites, hypochlorites etc than vegetative cells (Sella et al, 2014). They are also more UV resistant. Some spores can withstand many hours of direct surface UV radiation on Mars, including one strain still viable after 28 hours of simulated direct UV radiation in Mars simulation surface conditions (Galletta et al, 2010).

The iron oxide in the dust shields out UV and a microbe imbedded in a dust grain would have additional protection (Sagan et al, 1968). There isn't much research on survival of microbes in simulated wind blown martian dust, but what there is suggests it is possible for microbes to get attached to a dust particle and get blown in the winds (van Heereveld et al, 2017) (Osman et al 2008). The high wind speeds on Mars of 10 to 30 meters per second mean that the dust could travel hundreds of miles in twelve hours during a dust storm, and the storm would shield out much of the UV in addition to the shielding from individual grains.

The Mars dust storms could spread spores globally throughout the planet. Every decade or so, sometimes several times in a decade, it has global dust storms. These typically start in the south, during the southern spring or summer, encircle the planet in southern latitudes then extend north across the equator and can cover much of the planet (Shirley, 2015).

NASA have this as one of their many knowledge gaps for human extraterrestrial missions to Mars: 
- "Obviously, the current understanding of microbe survival in Mars dust environments remains uncertain and represents an important knowledge gap" (,Race et al, 2015. page 34)

\section{Sources of nitrogen on Mars}

Most of the necessities for microbial life, as we know it on Earth, are present on Mars.

There is plenty of carbon dioxide on Mars, 6 mbar on average. By comparison Earth has only 0.4 mbar at present, and over the last 600,000 years carbon dioxide fluctuated from below 0.2 mbar to above 0.25 mbar (Lüthi, 2008). Generally, carbon fixation is not a problem for photosynthetic life on Mars.

The native basalt has all the trace elements needed for life, and there is water available in the microhabitats [NEEDS CITE]

However there is only 0.2 mbar of nitrogen in Mars's atmosphere, compared to 780.90 mbar in Earth's atmosphere. Is this enough for nitrogen fixation?

Klinger et al tested two commonly used nitrogen fixing microbes in the lab, under conditions of low nitrogen levels, though full Earth atmospheric pressure. They found nitrogen fixation at 5 mbar, but no evidence below $1 \mathrm{mbar}$ (Klingler et al, 1989). In additional unpublished research they found a number of micro-organisms able to fix at a partial pressure of 0.2 mbar similar to modern Mars (Mancinelli, 1993).

Sakon et al used samples from the McMurdo dry valleys, and found cold tolerant nitrogen fixing microbes that fix nitrogen with the temperature and UV flux of Mars also simulated but still at full atmospheric pressure (Sakon et al, 2005) (Sakon et al, 2006). They proposed follow up experiments duplicating the Martian atmospheric conditions in a Mars simulation chamber to see if they could still fixate nitrogen.

They comment on the difficulty of testing psychrophiles in exploratory experiments, because the generation times are so slow. They used psychrotolerant microbes instead, easier to study and also cold tolerant. (Sakon et al, 2006). If Mars has life it may well include psychrophiles with slow generation times of centuries or longer similarly to Antarctic analogue habitats.

If biotic nitrogen fixation on present day Mars is impossible, naturally occuring nitrates may be a limiting factor for life, as suggested by (Bada et al, 2009).

Mars could have an abiotic nitrogen cycle with photochemically produced $\mathrm{HNO} 3$ fixed in thin $(0.2$ to $5 \mathrm{~nm})$ pure water metastable interfacial films. In this model the current atmosphere could support biomass incorporating an average of one kilogram of nitrogen per square meter (Boxe et al, 2012).

It can also have nitrates from past processes in early Mars. 
Curiosity has discovered nitrates in low concentrations ( 0.01-0.1 wt \% $\left.\mathrm{NO}_{3}\right)$ in Yellowknife bay drill sites, so they do exist on Mars (Stern et al, 2015). The upper end of the range here is the same as the lowest end of the range in the Atacama desert ( 0.1 to $\left.>1 \mathrm{wt} \% \mathrm{NO}_{3}\right)$

(Stern et al, 2015)

The researchers remark that concentrations are consistent with an impact generated nitrates reservoir from early Mars. If ammonia was detected this would support biological fixation but this is difficult for SAM to detect and easily destroyed by photochemistry. If indeed there isn't any reduced nitrogen, this could mean there never was enough life in early Mars to establish a cycle to return the nitrogen to the atmosphere in a nitrogen cycle. (Stern et al, 2015)

\section{All conditions for life may be present on or near the modern Mars surface}

In short, all the conditions for life may potentially be present on Mars in these suggested micro habitats. None of the conditions of ionizing radiation, low atmospheric pressure, high surface UV, the perchlorates and the diurnal temperature cycles currently rule out the possibility of native surface and near surface life on Mars.

Although we have not yet confirmed any habitats on Mars, part of the reason for this is that we have not yet sent any rovers there sufficiently sterilized to visit the best candidates. Curiosity is a few kilometers from a potential RSL (recurring slope lineae) and is not sufficiently sterilized to approach it closely to check it out.

It will take a fair bit of research to establish whether these or several other proposed near surface habitats exist. But if they do, the conditions there may be within the ranges tolerated by Earth life.

There may also be habitats that are outside the range for Earth life but can be tolerated by native martian life based on a novel biochemistry, perhaps with perchlorates or hydrogen peroxide as "antifreeze" (Schulze-Makuch et al, 2010)

\section{Could complete extinction be possible in the backwards direction, from space to Earth?}

It is certainly possible for an entire biosphere to be made extinct by new forms of life. None of our own early life or prebiotic chemistry survives. Hypothetically if we could introduce modern Earth life to the prebiotic or early life ocean on Earth, it seems unlikely that this early life would be able to compete with present day Earth life. This also could be the fate of prebiotic chemistry or some fragile early form of life in martian brines or a Europan ocean. 
So in the forward direction the worst case is complete erasure of the alien biology. In the reverse direction from Mars to Earth, what is the worst case? How much degradation of our biosphere is possible?

Let's start with hypothetical future astronauts on the martian surface. In the worst case, they could be attacked by martian life as soon as they get to the planet.

Legionnaires' disease, a disease of biofilms, is not especially adapted to humans, and uses the same methods to attack human lungs as it uses to attack a biofilm. This shows that humans could be vulnerable to diseases of martian biofilms (Alberts et al 2002), (Abdel-Nour et al 2013).

Martian microbes could also infect directly as new biofilms invading our lungs. $65 \%$ to $80 \%$ of human infections are associated with invasive biofilms (Lebeaux et al, 2013). Moreover, these biofilms are particularly antibiotic resistant.

If astronauts were infected with martian microbes or biofilms, would our antibiotics work with the possibly alien biochemistry of a martian biofilm invading our lungs? They target specific enzymes and processes within living cells based on Earth's biochemistry (Kapoor et al, 2017).

Let's take penicillin as an example of antibiotic action. Penicillin targets transpeptidase which is essential for the cross linking of transpeptidase in the final stage of cell wall synthesis to make rigid cell walls (Yocum et al, 1980). It does that by forming a highly stable penicilloyl-enzyme intermediate.

Would penicillin do anything to an alien biochemistry which lacks transpeptidase? One way that microbes develop resistance to this antibiotic is by evolving different enzymes that perform the same function in the cell (Gordon et al, 2000). An alien biochemistry might well have different enzymes already, through independent evolution.

In the same way all our antibiotics might be mystified by an alien biochemistry, with nothing to target.

We have also looked at opportunistic human pathogens, such as bacteria that are only infectious in wounds, with results similar to Tetanus (Warmflash et al 2007). Such pathogens could exist on Mars too. Exophiala jeanselmei is an endolithic species of yeast closely related to opportunistic human pathogens which could metabolize in Mars simulation conditions using only the night time humidity, without liquid water. Within seven days in the chamber, it recovered its pre-experimental level of metabolic activity with no signs of stress (Zakharova et al, 2014). The experiments were done with the Mars simulation chamber for German Aerospace (DLR),

We also looked at the example of BMAA which could be produced by alien microbiology and can be misincorporated to cause motor neurone disease. We will look at these and other 
indirect forms of action in more detail in the sections Accidental similarity of amino acids and Exotoxins, protoxins and allergens

There are many ways our astronauts could be harmed by martian life in the worst case.

Then on return to Earth, martian life could infect lifeforms other than humans. It could also harm microbial life through bacterial grazing and predation (Martin, 2002) (Velicer et al 2009). Martian life could kill Earth lifeforms though exotoxins such as Chyba's example of cows killed by algae in Lake Superior, and Warmflash et al's examples such as the anaerobe c. botulinum which can cause botulism (Warmflash et al 2007).

Could such a risk propagate back to Earth itself when the astronauts return or export materials to Earth, or even when an empty spaceship that delivered them to Mars returns to Earth?

This may seem like a scene from a science fiction book or movie. However if you feel we should reject it because of the appearance of science fiction, bear in mind that the idea of sending humans to Mars was itself science fiction a few decades ago. We are entering a future where we have to seriously consider what the real world outcomes might be in what used to be science fiction scenarios. Unlike the situation with science fiction, we don't get to write the script for this scene. Also we can't change the script if we don't like the outcome.

We have a responsibility to present and future generations to consider our prospects carefully. We have no experience of returning life from any other planet, and there is a distinct possibility that there is alien microbial life on Mars. Mars could have an alien biochemistry, perhaps not based on DNA, perhaps not using proteins, or DNA, or carbohydrates.

We don't yet have practical exobiology to guide us. So far we have no empirical data on what typically happens in collisions with a completely alien biochemistry. It could be harmless, so different that it is unable to attack us, but it could also work the other way around, that we are defenseless against its unfamiliar biochemistry.

Joshua Lederberg, who got his Nobel prize for his work on microbial genetics was a key figure in the early work on planetary protection (Scharf, 2016). He first began to give it his attention in 1957 (Lederberg, 1959). He put it like this:

"If Martian microorganisms ever make it here, will they be totally mystified and defeated by terrestrial metabolism, perhaps even before they challenge immune defenses? Or will they have a field day in light of our own total naivete in dealing with their "aggressins"? (Lederberg, 1999a)

"Whether a microorganism from Mars exists and could attack us is more conjectural. If so, it might be a zoonosis to beat all others. On the one hand, how could microbes from Mars be pathogenic for hosts on Earth when so many subtle adaptations are needed for any new organisms to come into a host and cause disease? On the other 
hand, microorganisms make little besides proteins and carbohydrates, and the human or other mammalian immune systems typically respond to peptides or carbohydrates produced by invading pathogens. Thus, although the hypothetical parasite from Mars is not adapted to live in a host from Earth, our immune systems are not equipped to cope with totally alien parasites: a conceptual impasse." (Lederberg, 1999b)

Our immune system and defenses are keyed to various chemicals produced by Earth life. such as peptides and carbohydrates. It's possible that Mars life doesn't use those chemicals.

In the best case (for us), the martian microbes are unable to make anything of terrestrial biochemistry and give up "totally mystified and defeated by terrestrial metabolism".

However, in the worst case, it's the other way around. Microbes from Mars could eat their way through Earth life. This time, it's our defense systems that are mystified. The microbes don't resemble Earth life and so our defenses wouldn't be able to recognize the attackers as life or do anything about it.

In this case, the microbes have a "field day in light of our own total naivete in dealing with their "aggressins".

(an "aggressin" is a substance inhibiting phagocytosis - is this how Lederberg is using the word here?)

Here is how John Rummel former NASA planetary protection officer expressed it when interviewed for NASA Astrobiology Magazine:

"After living in the dirt of Mars, a pathogen could see our bodies as a comparable host: they could treat us 'like dirt'. But to quote Donald Rumsfeld, we're dealing with unknown unknowns. It could be that even if the microbes lived inside us, they wouldn't do anything, it would just be this lump living inside you." (Mullen, 2003)

The physicist Claudius Gros looks at a clash of interpenetrating biospheres in his paper on a "Genesis project" to develop ecospheres on transiently habitable planets. However the same considerations could apply in the backwards direction from Mars to Earth.

He assumes that biological defense mechanisms evolve only when the threat is actually present and not just a theoretical possibility. He uses the same argument as Joshua Lederberg that the key to functioning of the immune system of multicelullar organisms, plants or animals, is recognition of "non-self". He puts it like this (Gros, 2016):

"How likely is it then, that 'non-self' recognition will work also for alien microbes?"

"Here we presume, that general evolutionary principles hold. Namely, that biological defense mechanisms evolve only when the threat is actually present and not just a 
theoretical possibility. Under this assumption the outlook for two clashing complex biospheres becomes quite dire."

"In the best case scenario the microbes of one of the biospheres will eat at first through the higher multicellular organisms of the other biosphere. Primitive multicellular organisms may however survive the onslaught through a strategy involving rapid reproduction and adaption. The overall extinction rates could then be kept, together with the respective recovery times, 1-10 Ma, to levels comparable to that of terrestrial mass extinction events."

"In the worst case scenario more or less all multicellular organism of the planet targeted for human settlement would be eradicated. The host planet would then be reduced to a microbial slush in a pre-cambrian state, with considerably prolonged recovery times. The leftovers of the terrestrial and the indigenous biospheres may coexist in the end in terms of 'shadow biospheres' "

That could happen in the reverse direction as well. If his argument is valid that Earth life is only evolved to protect against threats it has already encountered and not hypothetical threats from an alien microbe, then in the worst case, more or less all multicellular organisms on Earth could be eradicated by microbes brought back from the exoplanet.

It's the microbes that turn the other biosphere to slush in his scenario, not the higher lifeforms. So this could also happen with microbes returned from Mars as a worst case.

We will look later at the question of whether it's possible to estimate the chance of such an extreme outcome from an unsterilized sample return, suggesting a Drake equation approach may help clarify ideas. However, as with the Drake equation for estimating the prevalence of extraterrestrial civilizations - with no previous experience of biosphere collisions, there isn't much for astrobiologists to go on.

If this worst case is possible, this would still not be complete extinction of Earth life. Some Earth life might have resistance to martian life, or be able to evolve to resist it quickly. Other Earth life may live in conditions that the martian life can't tolerate, if its biochemistry is sufficiently different.

Also, our biosphere has us, as a thinking component with technology. Once the alien life is well understood, and we know how to keep it out, humans could survive in enclosed habitats on Earth and go outside also in suits that keep the alien life out. The habitats could have self contained biospheres based on plants grown for food, and oxygen, which in turn take up carbon dioxide and water from humans, similarly to the Russian BIOS-3 experiments (Salisbury et al, 1997).

If this had happened in the Apollo era we might not have had much chance of survival. But with the technology we have today, it seems survivable. 
Eventually, Earth's land area could be covered in habitats, and areas of its seas by sea steadings, reclaiming it for Earth life, in a process similar to "paraterraforming". With more efficient, self sustainable agriculture along the lines suggested for space habitats, such an Earth could support a population up to two orders of magnitude larger than it has today.

In the long term on this scenario, the alien biochemistry would hardly matter, at least for long term human survival and quality of life.

However, this worst case scenario would begin with a new mass extinction as great as any we have had in Earth's history, and in the worst case complete extinction of multicellular life outside of the habitats. In the near term, this would lead to temporary widespread hardship, and severe degradation of the environment of Earth for humans.

\section{The need for a vigorous in situ astrobiological search}

It's generally agreed by astrobiologists that a survey can't be done with a sample return mission alone. At our current stage of understanding of Mars a sample returned without prior in situ detection of clear biosignatures is regarded as more of a technology demo than an approach that can answer central questions in the field (Paige, 2000), (Bada et al, 2009), (Davila et al, 2010) (Schulze-Makuch, 2015).

Detection of organics in situ would not be sufficient. According to calculations, Mars should have $60 \mathrm{ppm}$ of organics from organics deposited into the regolith by interplanetary dust, meteorites or comets. That figure is obtained by averaging the infall over its entire surface to a depth of a hundred meters. This does not include any indigenous organics produced by life or abiotic processes.

Surface processes would degrade some of it, but much of it may still be present. The martian meteorites have levels of organics roughly consistent with that estimate, and come from a few meters below the surface of Mars. This is below the influence of surface chemistry and the worst of the ionization effects (Goetz et al, 2016).

Some of these organics from meteorites may mimic biosignatures. The primordial chondrite meteorite GRA95229 collected in Antarctica had chiral excesses ranging from $+31.6 \%$ for a-AIB to $+50.5 \%$ for isovaline, while another meteorite EET92042 had excesses from $+31.8 \%$ for glycine to $+49.9 \%$ for L-alanine. It's thought that these excesses are extraterrestrial and not due to contamination by Earth life (Martins et al, 2007). A recent study of sugars in the Murchison meteorite, along with others, found excesses of up to $55 \%$ for threonic acid, a four carbon sugar acid. One of the 5 carbon sugar acids had an even more dramatic excess of up to $82 \%$. These excesses affected biologically rare sugar acids, which the researchers see as evidence that these excesses are not due to contamination from Earth life (Cooper et al, 2016). Early life on Mars could also predate homochirality (Brazil, 2015). In addition non biological processes can produce biochemicals including RNA (Trinks et al, 2005). 
The Tissint martian meteorite has organics with a carbon 13 depletion signature, another candidate biosignature, which is not considered to be proof of a biological origin in that case (Lin et al, 2014).

NASA's sample caching rover Mars 2020 would detect all these meteorites as high priority samples to return to Earth. Such samples would be as ambiguous for astrobiology as the martian meteorites we have already (Bada et al, 2009).

A vigorous in situ astrobiological exploration would involve sample returns for more detailed astrobiological investigation, but only after clear signs of life have already been discovered in situ. Our modern understanding of Mars is complex and varied. Sub-meter resolution imaging from orbit as well as in situ investigations by our landers and rovers have turned up many surprises, some of astrobiological significance. A preliminary astrobiological survey would require searches of many features in large areas around a dozen or more landing sites. Astrobiologists also stress that such a rover has to have the capability to drill to some depth.

Although the current sample return program has been motivated as part of the search for life, its primary function seems to be geochemical. For reasons of cost, the sample return tubes for Mars 2020 will not be sterilized completely. For any organics in the sample, then as with the martian meteorites, there would remain the issue of disentangling native organics from contamination by Earth based organics. They also permit a small probability of a viable microbe remaining in one of the tubes.

This means that if we were to detect a viable spore or dormant microbe in one of the samples, and it resembed one of the $0.0001 \%$ of microbial species already sequenced we might deduce it was contamination. It would be hard to prove it to be native life if it resembles Earth biochemistry and isn't in our genetic databases. The chance of this is remote perhaps, but not impossible if microbial spores on Mars are as pervasive as the ambiguous Viking experiments suggested.

Although Mars 2020 won't be able to detect life in situ, it is still possible that the sample return might return life. The remote chance for this is the reason that so many precautions would be needed. But because it is not sterilized, even in that case we would be quite likely to be unable to prove conclusively that it is martian life rather than contamination from Earth, at least, not if it is a distant cousin or closely resembles Earth life in its biochemistry.

\section{List of astrobiological instruments for in situ searches}

There are many instruments we can send to Mars to search for life in situ. They would search for many biologically relevant molecules, using microfluidic supercritical water extraction, so sensitive they could detect a single amino acid in a one gram sample (Schirber, 2013) (Noell et al, 2016). They would also use polyclonal antibodies to search for the organics of life (Parro et al, 2011). 
They would also add the samples to culture media and look for evidence of metabolic processes, and check for chiral preferences by detection of carbon 14 in emitted methane or carbon dioxide (Anbar et al, 2012). They can also detect redox reactions directly by measuring the electrons and protons they liberate. This is sensitive to small numbers of microbes and has the advantage it could detect life even if not based on carbon or any form of conventional chemistry we know of (Abrevaya et al, 2010). Both these techniques do not require the life to reproduce, only metabolize. They can be used for slowly metabolizing life with generation times of months or years, as is likely in conditions such as those common on Mars.

They would use specialist imaging including fluorescence imaging_(Hand et al, 2017),

They could use microscopic imaging of autofluorescence as well as search microscopically for mobility of the microscopes themselves. The smallest microbes are too small to swim because they get jostled about too much by brownian motion. But microbes can swim if they are larger than 0.6 microns, and we often see microbes larger than 0.8 microns swimming on Earth. That's far larger than the limit of optical resolution of 0.2 microns. Also, they are often mobile even in very cold dry conditions on Earth. For a typical CCD camera used for microbial imaging, and typical microbe concentration of Earth seas, of 100,000 to a million microbes per millilitre, then you would get an average of much less than one cell per image. So, it would help if you have some way to concentrate the population of microbes (Nadeau et al, 2016). Another approach is to use an off-axis holographic microscope, which lets you choose the focal plane after the image is captured. Lindensmith et al were able to use such an instrument to detect active prokaryotes at $800 \mathrm{~nm}$ resolution through their mobility in sea ice imaging samples from near Nuuk in Greenland (Lindensmith et al, 2016).

Raman spectroscopy, such as will be used with Mars 2020 and ExoMars can help with non destructive detection of organics. However, it comes with a drawback especially for the search of past life. The best chance of finding biosignatures of past life is in organics that are mixed into clay and other minerals altered by water on Mars, because they get incorporated into the minerals in a way that protects them from deterioration, However experiments show that this can make it hard for Raman Spectroscopy to disentangle the organic from the mineral it's embedded in (Hamilton, 2016).

They could use Raman microspectroscopy which synchronizes Raman with an optical microscope and uses laser light focused in the same optical plane as the microscope (Hand et al, 2017). ,

One possibility is the discovery of early cells, smaller than the optical resolution limit. This makes techniques of superresolution optical microscopy of special interest, a field of active and rapid development (Perkel 2016). They could use near-field optical microscopy (Board, 2007 page 72), and superresolution optical microscopy (Hand et al, 2017).

They could also use a miniature variable pressure electron microscope capable of in situ chemical analysis as well as imaging (Gaskin et al, 2012). 
With SETG then the instrument suite can also include an end to end gene sequencer (Mojarro et al, 2016).

Some of those approaches, for instance direct optical observation of motile microbes, would give reasonably conclusive detection of life.

However, it's generally agreed that ideally an astrobiological in situ life detection suite should have several simultaneous methods of searching for life, as for Viking. These modern candidate instruments to send to Mars are small in size, and low mass, indeed many are "labs on a chip". They also have low power requirements, and an entire suite including several such instruments would mass a few kilograms.

\section{Well beyond the capabilities of currently planned rovers - preparation for humans on Mars prioritized over astrobiology}

All this is well beyond the capabilities of Mars 2020, which doesn't have any dedicated in situ astrobiological instruments. It would have been easy to add such a capability.

They prioritize preparing for an eventual human landing over in situ searches for life. Mars 2020 will carry MOXIE to test in situ oxygen production for a human mission on the Mars surface. It has a payload mass $15 \mathrm{~kg}$ (Hartvigsen et al, 2017), enough for 3 - 6 astrobiology life detector instruments, and does nothing except generate oxygen which is then vented into the Mars atmosphere. The Mars 2020 rover doesn't need oxygen. And MOXIE doesn't need to move.

This search also has to involve drilling or digging. This might only need to be to a depth of a few centimeters for most of the habitats for active present day near surface life. For dormant but still viable life, we need to drill to a meter or so. For past life we need to drill to several meters with robotic self hammering moles such as the one used for the heat flow probe on the Insight lander (able to penetrate five meters). This is the only way to reach layers of clay, salts, ice and other deposits deep enough to preserve the signature of ancient life, unless we are lucky enough to find the samples we are interested in recently excavated naturally by a meteorite impact or wind erosion. Any ancient biosignatures in shallower layers would be erased by the constant flux of penetrating cosmic radiation.

Such signals are also likely to be hard to spot, especially if Mars never developed photosynthetic life, with the expected strong signal from abiotic organics, and the difficulty of preserving ancient life in the super oxygenated surface conditions and the ionizing radiation .(Westall et al, 2013) (Westall et al, 2015).

To do this we need to resume in situ astrobiological explorations, paused for a quarter of a century. The two Viking missions remain our first and only dedicated astrobiological missions to date. They gave ambiguous results because of the unexpectedly reactive 
superoxygenated soil chemistry. These have not been resolved to this day. The debate was reopened with Joseph Miller's discovery of rhythms in the data resembling circadian rhythms, offset from temperature fluctuations by a hard to explain 2 hours (Miller et al, 2002). Researchers soon devised follow up experiments that we can send to resolve this. The reason the results are still ambiguous to this day is because these experiments have never been sent to Mars.

Perhaps the top non biological candidate at present is the reaction studied by Quinn et all in 2013. They suggested that the perchlorates were decomposed to hypochlorite $\left(\mathrm{ClO}^{-}\right)$, trapped oxygen, and chlorine dioxide. Then the release of oxygen from the trapped salts, plus the reaction of the hypochlorite with the amino acids may explain the results (Quinn et al, 2013).

However, there are many things to explain, particularly the two hour offset of the circadian rhythms, that two of the labeled release experiments got inactivated after storage in darkness for several months, and that the activity of the soil is significantly reduced at $50{ }^{\circ} \mathrm{C}$. Levin and Straat in a paper published in 2016 review this and other proposals (Levin at al, 2016).

We can expect some of the astrobiological missions to yield initially ambiguous results as for Viking, since after all, it is our first ever astrobiological search anywhere. We can't expect to get everything right at the first try. Scientific experiments often lead to ambiguous leads that need to be clarified. But with a vigorous program of exploration this should not be a problem.

The Europa Lander report set the requirement (Hand et al, 2017). ,

- "Life-detection experiments should provide valuable information regardless of the biology results"

We need to get away from this approach if we are going to have really serious in situ searches in our solar system. Many of the astrobiological instruments mentioned in this section could not be sent with that requirement, or if they had some abiotic value, would not rank high if judged according to their benefit for geochemical studies.

We should treat uninhabited habitats as an interesting potential discovery in their own right (Cockell, 2014). Rocks from volcanoes, soon after they cool down, are inhabitable but uninhabited. Some regions of our harshest deserts don't have life, for instance gypsum pillars in the very driest areas of the Atacama desert, but that's because they are too dry for any Earth life. Just possibly Don Juan lake in Antarctica is uninhabited too - it has microbes but they probably don't grow there, but if so, it's because it is too salty for Earth life.

If we only search for life once we know it is there, how can we expect to find life, unless it is an easily recognizable biofossil or macroscopic lifeform? A null result is of scientific value and can help focus the search (Kite et al, 2018). In addition a potential habitat with no life in it is also of scientific interest in itself 
If we do discover a potential habitat in space, one which on Earth would be colonized by microbes, and get a null result from the life detection experiments, this might suggest an uninhabited habitat in space. This should be treated as a major discovery in its own right. It would be the first discovery in our solar system of what may be a common situation in our galaxy.

Places that are outside the normal range for Earth life are of astrobiological interest too, because we don't know of the limits for non Earth life. Is there life on Mars that can live in such habitats though Earth life can't? The answer to that is also interesting both ways, whether we find it, or don't. If Earth life hasn't adapted to those conditions, maybe Mars life has?

So let's send our astrobiological experiments to these places, designed to look for life, or to rule it out, and let's not add any requirement that they also produce valuable geological information. And let's see if there is life there - either way. And if there isn't life in what seem to be habitable brines - especially if it also has organics - well study it with our astrobiological and non astrobiological instruments, look for prebiotic chemistry, and try to find out more!

Discovery of an uninhabited habitat can help us learn about abiotic and prebiotic chemistry on other planets and it would then be a scientifically significant part of these observations to show that the chemistry found in the sample is not due to life

\section{Top candidate sites for an astrobiological investigation of Mars}

Before astrobiologists can be expected to give a reasonable assessment of the planetary protection status of Mars they would need to do at least a preliminary survey of the present day life there as well as past life. As we'll see later, this survey could go quickly if this survey was given a fraction of the resources expected for a human mission to Mars. But we need to be clear about how complex and involved such a survey would be.

It is a big ask for astrobiologists, to require them to assess the planetary protection status of such a complex planet. The aim of this and the next section is not to be comprehensive, but rather, to give an idea of the diversity of approaches needed to make a first sketchy astrobiological survey of Mars, to help inform decisions for future planetary protection strategies.

There are many places to search for life in the complex landscape of Mars. These include the Recurring Slope Lineae on the walls of Valles Marineris and at higher latitudes which occur on steep slopes (McEwen, 2011)(Olga et al, 2015) (Edwards et al, 2016) (Dundas et al, 2017) (Stillman et al, 2017). One way to explore steep slopes on Mars is to use cliff-bots one rover goes to the head of the slope and then rapels on a cable tethered to either one or two rovers that remain at the top of the cliff (Abad-Manterola et al, 2009) (Kerber et al, 2016) (Paulsen et al, 2005) (Stroupe et al 2007).

Then there's the Hellas basin which has the highest atmospheric pressure on Mars, where at times, conditions of pressure and temperature are sometimes above the triple point for 
water, so that liquid water is briefly stable on the surface though close to boiling point. It may be an optimal place to look for life that might rely on $100 \%$ night time humidity for water, or that relies on melting of the morning frosts. In all, $29 \%$ of the surface of Mars is at least occasionally above the triple point for water and fresh water could form there briefly if there were conditions that lead to it ice forming and then warming. Hellas basin is most favourable with conditions for liquid water for 60 sols of the year. Next most favourable is the Amazonis region where those favourable conditions are encountered for 27 sols of the year (Haberle et al, 2001)

Then Richardson' crater in the southern polar regions to examine the dry ice geysers - and especially the flow-like features that spread down slopes from the debris of the geysers at meters a day in late spring, and generally to look for dust and meteorites in the polar ice caps to see if there is any water around them, as happens in similar conditions in Antarctica below clear ice. (Möhlmann, 2010c). (Losiak et al, 2011) (Martínez et al, 2013)

Then there are the ice / salt plains of the ancient northern ocean bed where the Phoenix lander landed. These are an ideal place to search for the droplets that form when salt overlays ice as in the experiment by Nilton Renno's team. (Fischer et al, 2014)

We also need to search for life in the ice and salt below the surface, kept dormant since Mars was more habitable last time its atmosphere thickened temporarily as it does every few million years due to variations in its orbital eccentricity and axial tilt, and the northern planes are a suitable site to drill deep to search for dormant life in the ice as for Chris McKay's Icebreaker Life mission (McKay et al, 2013).

We can search for caves, which would be of many different types on Mars, as varied as on Earth and some formed by processes unique to Mars involving dry ice, or rare on Earth involving sulfuric acid. Most of them won't be easy to spot from orbit.

Penelope Boston lists some of the types of cave possible on Mars (Boston, 2010)

1. Solutional caves (e.g. on Earth, caves in limestone and other materials that can be dissolved, either through acid, or water). The abundance of sulfur on Mars may make sulfuric acid caves more common than they are on Mars.

2. Melt caves (e.g. lava tubes and glacier caves)

3. Fracture caves (e.g. due to faulting)

4. Erosional caves (e.g. wind scoured caves, and coastal caves eroded by the seas on ancient Mars)

5. Suffosional caves - a rare type of cave on the Earth, where fine particles are moved by water, leaving the larger particles behind - so the rock does not dissolve, just the fine particles are removed.

6. Sublimational caves caused by dry ice and ordinary ice subliming directly into the atmosphere (a process that doesn't occur on Earth). 


\section{Candidate sites for past life}

Understanding the past history of Mars will also help with planetary protection assessment by uncovering details of the evolutionary history behind any present day life. It can also be done at an earlier stage when rovers are not yet sufficiently sterilized to approach the special regions, but are sufficiently sterilized to explore regions with no local habitats for Earth life.

There are as many places to explore for past life. They include the ancient hydrothermal vents of the Volcanic cone in Nili Patera Caldera. which must originally have been warm and wet in the Noachian period (Fawdon et al, 2015) (Skok et al, 2010), Nili Fossae which has evidence of multiple hydrothermal episodes in the early Noachian (Ehlman et al, 2009), and the fossil hydrothermal vent turned up by the Spirit rover at Home Plate in Gusev crater, as well as the carbonates and hydrated silicates on the peak of Leighton crater excavated from $6 \mathrm{~km}$ below the surface suggesting it was a Noachian period hydrothermal vent habitat (Michalski et al, 2010a)

The Melas Chasma in Valles Marineres, of interest as a unique section through the upper few kilometers of the crust of Mars (Flahaut et al, 2012).

Elysium Planitia is interesting as a deep area of what may be a frozen sea, ancient ice trapped below surface layers, and unusually, still present in the equatorial region of Mars (Murray et al, 2005)

The Noachian period Eberswalde delta, rich in clays and a great place to find preserved organics from the original river that flowed into the sea, and a candidate site for Curiosity (Pondrelli et al, 2008).

The nearby Holden Crater below was another candidate for Curiosity as well as for Mars 2020 , because it also has many features suggesting liquid water in the past, and because of its mineral diversity. With faster rovers maybe we can visit both.

Marwth Vallis, the third site proposed for Curiosity, in the search for ancient life on Mars, an area of Mars with layered clay deposits, also happens to be close to an easy place to land, which could preserve ancient organics and micro-organisms could be preserved within those clays which preserve organics well on Earth (other good places to look for them are in the salt flats). It's on the boundary between the Southern highlands and the northern lowlands, where the land slopes steeply. It has layered cliffs which contain clay, one of the best materials for preserving ancient organics. There are many sites on Mars with clay deposits, but the interesting thing about these is that they are in clear stratified layers as beloved by geologists trying to work out sequences of geological events (Michalski et al, 2010b).

Recent craters would remove the surface dust, excavate materials from below the surface, and expose the subsurface, make it easier for our rovers to examine them in pristine condition (Lucchetti et al, 2104) 
The Mars ice caps have layered deposits which surely have trapped bubbles preserving fluctuations in the Mars atmosphere composition. In 1976-8, a group of scientists in Purdue developed an idea for an ice core sample return from the Martian south polar ice cap. The idea was to send a core driller to Mars to dig a 50 meter core and then return this to Earth (Staehle et al, 2013). More recently, a team at Cornell university is working ona Martian Cryobot to explore the layers of the Martian ice caps by a team at Cornell university (Aharonson)

The most interesting area perhaps is the area around the polar ice cap, the Dorsa Augusta, of late Noachian, early Hesperian period. These ridges are interpreted as eskers in the Dorsa Argentae region - places where water flowed under the ice in the late Noachian period (Fastook et al, 2012)

Recent volcanism formed several lakes 210 million years ago on one of the flanks of Arsia Mons. That's relatively recent in geological terms, so recent that there seems no reason why it couldn't happen again today. It probably formed two lakes with around 40 cubic kilometers of water each, and a third one of 20 cubic kilometers of water. They probably stayed liquid for hundreds, or even thousands of years (Scanlon et al, 2014)

Much of this can be done remotely from Earth initially. NASA planned dedicated satellite om 2022 with optical broadband communication with Earth, and $30{ }^{\circ} \mathrm{Cm}$ resolution imaging of Mars. It was planned to have a bandwidth of 800 gigabits a day, enough to return more than all the 500 gigabits of data from the Pluto New Horizons mission in a single day (Reichhardt, 2016). However they have canceled this in order to focus their funding on the Mars sample return mission (Smith, 2016).

We need high bandwidth communications to return high resolution images from Mars from the surface and from orbit. It would also greatly speed up the science done on Mars. At present the light speed delay is not the bottleneck, it's the bandwidth. They typically communicate once a day. Our rovers could be on distant Sedna, and we would hardly notice any difference due to the extra light speed delay of 12 hours.

Once we have high bandwidth, the next bottleneck is the latency and for this humans in orbit around Mars could increase the pace of discovery again. In this way we can aim for several orders of magnitude increases in the pace of exploration and discovery from Mars.

\section{Is transfer of life possible from Mars to Earth?}

Most of the papers on transfer of life between the planets Earth and Mars focus on the direction from Mars to Earth. That is the easiest direction for transfer of life, especially in the early solar system when Mars had seas. At $5 \mathrm{~km} / \mathrm{sec}$, its escape velocity is less than half that of Earth's. This leads to a lower shock of ejection, for materials originating in a zone a few meters below ground level on Mars. 
One example organism used in these studies is the polyextremophile Chroococcidiopsis. It is one of our top candidates for photosynthetic life that might be able to survive on Mars today, as a primary producer, requiring little more than sunlight, trace elements, nitrogen and water, and able to use high humidity in the atmosphere as a source of water, also UV resistant and able to survive partial shade conditions on the Martian surface, or protected by a thin layer of dust.

Although Chroococcidiopsis is classified as a single species, it reproduces asexually (and can also share gene fragments even with completely unrelated life forms via gene transfer) and there are many varieties of it. For instance desert varieties have especially high resistance to UV light, and lose some of that resistance when brought into the laboratory.

However, as far as is known, chroococcidiopsis doesn't have the capability to form hardy spores or dormant resting states to resist the shock of ejection from Earth. It has to survive any transfer between planets as an ordinary cell.

Typically ejection from Mars imparts a shock of 5 - $55 \mathrm{GPa}$, based on analysis of the Mars meteorites (Nicholson, 2009). Chroococcidiopsis can survive up to $10 \mathrm{GPa}$. The microbe Bacillus subtilis and the lichen Xanthoria elegans survived up to $45 \mathrm{GPa}$ (Stöffler et al, 2007)

The more highly shocked meteorites had the shortest transit times, as predicted by modeling, since the most shocked also have the highest residual velocities, and so can reach Earth sooner.

ALH84001 was heated by $100 \mathrm{C}$, to a temperature less than $40{ }^{\circ} \mathrm{C}$ (because it retained magnetism that would be destroyed at that temperature), and a shock of $32 \mathrm{GPa}$ (Fritz et al, page 1402).

The two least shocked martian meteorites were Lafayette and Y-000593 with shock of 5-14 Gpa. Both had ejection ages (time spent in transit) of 12 million years. The shortest ejection age is for EET 79001 with an ejection shock of around $36 \mathrm{GPa}$ and a temperature increase of around $560 \mathrm{C}(560 \pm 120)$ (Fritz et al, table 1 page 1401)

If Chroococcidiopsis can get onto a meteorite and survive the shock of ejection, Chroococcidiopsis can withstand many of the stresses of panspermia. It can survive the low temperatures and vacuum of space and the UV radiation at least for 1.5 years, and probably much longer, tested in experiments flown on the exterior of the ISS_de La Torre, 2010). Also, it can be protected from UV radiation by a thin layer of a few $\mathrm{mm}$ of rock.

The main challenge for photosynthetic life is that it has to survive the fireball of entry into Earth's atmosphere. (Cockell, 2008)

This happens at a minimum re-entry speed of over 11 kilometers per second. Typically $10 \%$ - $20 \%$ of the radius of a hand sized rock ablates away. The rock also has to be larger than 20 centimeters in diameter to avoid heating up to $100^{\circ} \mathrm{C}$ all the way through to the center (which would sterilize it of photosynthetic life). Since photosynthetic microbes normally grow 
at most a few millimeters below the surface of a rock, it's clear already that it's going to be tricky for it to survive entry into our atmosphere.

Cockell did an experiment in which he inculcated some Chroococcidiopsis into an artificial gneiss rock, at a depth where it could be expected to grow naturally. He fixed the rock into a heat shield of a re-entry capsule launched by a Soyuz rocket. None of the microbes survived re-entry, nor did any of their biomolecules (Cockell, 2008).

The interior of a rock can be better protected. The interior of ALH84001 never got hotter than $40^{\circ} \mathrm{C}$ during entry into our atmosphere (Weiss et al, 2000). But how does the photosynthetic life get deep into a martian rock? It can flourish in cracks, if light filters in through them - but that also would give cracks that channel hot gases into the interior of the rock during re-entry.

Another factor is cosmic radiation. This sterilizes the surface of a meteorite to a depth of 2 $\mathrm{cm}$ within 100,000 years by breaking up the nucleic acids. That's below the maximum depth you'd expect to find photosynthetic life in normal circumstances, even in fine cracks.

It follows that nearly all rocks that get to Earth from Mars will be sterile of photosynthetic life by the time they get there, even not taking account of the ablation of the surface of the meteorite.

It is theoretically possible for some rocks to get to Earth as soon as ten years after ejection from Mars. But most take between a hundred thousand and ten million years to get there. Assuming a maximum ejection velocity of $6 \mathrm{~km} / \mathrm{sec}$, then in a simulation with 2100 particles, incorporating the gravitational effects of all the planets from Venus through to Neptune, most took over 100,000 years in transit. The fastest transfer in the simulation was 16,000 years (Gladman et al, 1996).

Charles Cockell's concludes that it might not be impossible for photosynthetic life to get to Earth from Mars, but it would need a rather extraordinary combination of events (Cockell. 2008):

"Thus, the planetary exchange of photosynthesis might not be impossible, but quite specific physical situations and/or evolutionary innovations are required to create conditions where a photosynthetic organism happens to be buried deep within a rock during ejection to survive atmospheric transit."

His final conclusion is that photosynthetic life has the potential to make dramatic changes to a planet, but that this transfer of photosynthetic life is less likely than for heterotrophs (which use organic carbon) or chemotrophs (which use chemical reactions as a source of energy and synthesize all their organics from carbon dioxide, living in places such as hydrothermal vents).

Could there be other forms of photosynthetic life on Mars that could survive the shock of ejection more easily than chroococcidiopsis? 
In one paper, samples of a marine photosynthetic algae nannochloropsis oculata frozen in ice were able to survive $6.93 \mathrm{~km} / \mathrm{sec}$ impacts into water with approximate shock pressure of $40 \mathrm{GPa}$ (Pasini, 2014).

However this only solves some of the issues. The life would still be ablated by the fireball of re-entry as well as most of it sterilized by the ionizing radiation of the transfer.

Perhaps transfer of photosynthetic life would be easier in early Mars with porous rocks on a sea or lake bed that could be infused with shock resistant photosynthetic marine life through ocean and lake currents, and then transferred to Earth before they die? [ANY CITES FOR THIS?]

The meteorites we have from Mars come from at least 3 meters below the surface in the southern uplands. We know this from the concentrations of Krypton 80 produced as a result of the secondary neutron flux of cosmic rays in the martian regolith. This should have produced Krypton 80 through neutron capture from Bromine 79. (Head et al, 2002, page 1355).

- Many of the proposed habitats are in the surface layers of dirt, ice and salts. So would any of that get transferred to Earth after an asteroid impact on Mars?

- Other habitats are just millimeters below the surface of rocks. These layers would ablate away during entry into the Earth's atmosphere

- Some rocks may have life beneath the surface and others not by analogy with similar conditions on Earth. Some areas may have the right conditions, mix of salts, humidity, for the liquid brines below the surface to have life and others not.

- Life on Mars could be extremely localized to only a few square kilometers over the entire planet, for instance, only to the RSL's, or only above geological hot spots. This would make it unlikely that a meteorite ejected from present day or geologically recent Mars will sample one of those habitats.

The largest pre-atmospheric sizes for meteorites in our collections are 20 to $30 \mathrm{~cm}$ and the largest possible for impacts for the last 20 million years is about 2 meters (Eugster et al, 2002)

(air thinner and the glancing impacts that can send material to Earth send material from deep below the surface). This is one of the least likely locations for present day life on Mars]

\section{Has life from Mars caused mass extinctions on Earth in the past? Was the Great Oxygenation Event such an extinction?}

It's possible that there has been life transferred from Mars to Earth. This does not mean that it is safe to return material from Mars. 
The best time for transfer was in the early solar system, and transfer in recent times is unlikely since the martian meteorites come from what may be a largely lifeless zone on modern Mars a few meters below the surface (from both impact modeling and study of the meteorites themselves).

The National Research Council looked into this question in their "Assessment of Planetary Protection Requirements for a Mars Sample Return". They don't go into their reasoning in any detail, but they were unable to rule out the possibility that life from Mars could have caused mass extinctions on Earth in the past (Board et al, 2009:48).

How could a microbe transferred to Earth from Mars cause a mass extinction?

Let's look at the Great Oxygenation Event, which could potentially be caused by a martian microbe. Chroococcidiopsis is an ancient microbe that may be one of the ones that caused the oxygenation of our atmosphere. One suggestion to explain the ionizing radiation resistance of Chroococcidiopsis is that it originated on Mars (Pavlov et al, 2006).

Whether it caused mass extinction of microbial life is a matter of debate as many anaerobic habitats survived. Microbes are numerous and evolve quickly. Early anaerobes may have instead retreated to anaerobic habitats as obligate anaerobes, which we still have today (Lane, 2015). The Great Oxygen Event might have forced rapid evolution rather than extinction.

However, there is evidence for exceptionally large sulfur reducing bacteria from this time, 20 to 265 microns in size. They also occasionally occur in short chains of cells. This may be part of a diverse ecosystem that predated the GOE (Czaja et al, 2016).

Could this anaerobic ecosystem have included multicellular life? The metabolic rate required by an organism depends on its size with larger ones requiring a higher metabolic rate in a complex relationship (super linear power law for prokaryotes, linear for protists, sublinear for metazoans) (DeLong et al, 2010).

Some $1 \mathrm{~mm}$ scale anaerobic metazoans use the less efficient hydrogenozomes which don't require oxygen and produce hydrogen as a waste product (Danovaro et al, 2010). These seem to have evolved from mitochondria, through multiple times through convergent evolution (Yarlett et al, 2005). However it suggests the possibility of anaerobic multicelluarity.

A genetic analysis of modern cyanobacteria suggests that the majority of species evolved from multicellular ancestors. This may push the origin of primitive multicellularity in cyanobacteria back as far as the great oxygenation event (Schirrmeister, 2011). Fossil multicellular life in strands decimeters long has been found dating back to at least 1,560 million years ago (Zhu, 2016) 
None of this is definite evidence of multicellular life predating the GOE. We can't yet know if the GOE caused the extinction of any multicellular life, but it does seem not impossible that it had major impacts on a diverse ecosystem which we know did include large complex sulfur reducing anerobic microbes over a quarter of a millimeter in diameter.

Whether or not the GOE was a mass extinction, and whether or not it was caused by martian microbes, there are many other confirmed later mass extinctions in the fossil record. In many cases the cause is not known or debated, and the possibility can't be ruled out that some of those also were caused in some way by microbial life that got here from Mars.

There isn't enough evidence yet to know if the great oxygen event or any of these other events did cause a mass extinction. However the hypothetical example of chroococcidiopsis as a potential cause of oxygenation of the Earth's atmosphere does seem to suggest it is a possibility that a microbe from another planet could cause a mass extinction on Earth.

\section{Transfer of life is hard in the direction from Earth to Mars - and especially so for photosynthetic life (MID EDIT)}

\section{(WORK IN PROGRESS)}

Though life on Mars is likely to be able tolerate oxygen in surface habitats, introducing photosynthetic life that competes with native photosynthetic life based on different principles could be a major change. It's also possible that native martian life never developed photosynthesis, since it may not have had liquid water for extended periods of time and its oceans may have been frequently covered in ice (Westall et al, 2013)..

So, could photosynthetic life be identical on Earth and Mars? Or indeed, could any photosynthetic life have got from Earth to Mars already?

To take an example, the polyextremophile Chroococcidiopsis is potentially adapted to survive over much of the Mars surface using just the night time humidity according to the DLR experiments. If so, it could, potentially, have a huge effect on Mars if it is not there already.

The shock would probably be greater for an ejection able to send photosynthetic life from Earth to Mars.

So, would chroococcidiopsis get there on meteorites? Is it already there perhaps? Or might it perhaps have come to Earth from Mars? Or must any photosynthetic life on Mars be native to the planet?

The UK astrobiologist Charles Cockell looked at the possibility of transpermia for both single celled and multicellular photosynthetic life (such as lichens) (Cockell, 2008) 
He found out that it is possible, but difficult and rather unlikely, except perhaps in the early solar system.

into the interior of the rock. Cracks like that would also be places where the rocks are quite likely to break apart during ejection from Mars or re-entry to Earth.

Also, chroococcidiopsis is rather susceptible to shock of ejection from Mars. It's killed at only $10 \mathrm{GPa}$ (Nicholson, 2009). Typically ejection from Mars requires 5 - $55 \mathrm{GPa}$, based on analysis of the Mars meteorites. So that suggests that it can just survive ejection from Mars at the lower end of impact shock levels. Lichens manage somewhat better here. But that's not much use if they can't survive entry into the Earth's atmosphere when they get here.

In the other direction, from Earth to Mars, with escape velocity $11.2 \mathrm{~km} / \mathrm{sec}$, so more than twice the escape velocity of Mars of $5.02 \mathrm{~km} / \mathrm{sec}$, the higher shock levels would make it very hard for Chroococcidiopsis to survive ejection.

The speed of the ejecta never exceeds half the impact velocity. So, for any ejecta to escape Earth the impact velocity has to be over $22 \mathrm{~km} / \mathrm{sec}$. About half of asteroid impacts and most comet impacts exceed this (Gladman et al, 2005, page 8).

You can work out scenarios by which photosynthetic life could get from Earth to Mars. For instance, make the original impact into an ocean, and then the photosynthetic life gets forced into rock as a result of the impact and that rock, now impregnated with photosynthetic life to some depth, now gets ejected into space.

Lichens and some photosynthetic algae are more resistant to the shock than Chroococcidiopsis.

Also, what is the minimum shock of ejection for the larger impacts needed for the Earth to Mars direction, for a really huge impact like the Chicxulub one? When the Martian meteorites were discovered, researchers were surprised to find that they were so lightly shocked. But this is something that is now well understood, first explained in 1984. The low levels of shock arises from interaction between the shock wave moving away from the forming crater and a reflected shock wave moving backwards. The shock moving back is 180 degrees out of phase so the two shock waves cancel, creating a lightly shocked "spall" zone where the two interact.

The spall zone depth is proportional to the radius of the impactor, so a large impactor would have a thicker spall zone (summarized in section 2 of this paper, original paper here).

Some of the ejecta would survive shock of less than $1 \mathrm{GPa}$ according to (Mileikowsky et al, 2000)

mi

https://arxiv.org/ftp/arxiv/papers/1311/1311.2558.pdf 
79 particles from Earth to Mars, shortest time for transfer 112,689 years.

1,048 from Mars to Earth, shortest time for transfer 281,754 years (Worth et al, 2013)

If Mars never developed photosynthesis, and never evolved it, then any photosynthetic life introduced on our spacecraft could potentially have a major impact. It could make some species of native Mars life extinct (if they exist) by competing with it for resources or shading it, or by creating chemical byproducts that make the habitats inhospitable to other lifeforms already there. Of course it could also serve as food for the right kinds of organisms there. Either way, it would be a major change in the microbial ecology and the ecology of micro habitats of Mars. If it was was able to survive on the surface just using night time humidity, as in the DLR experiments, and spread over large areas of Mars, perhaps it could also reduce the carbon dioxide levels in the atmosphere slightly too, cooling down the planet more than it is already.

Or if not now, it could have that effect in the future when Mars' climate warms up as it does from time to time in chaotic fashion. It could also act to counteract attempts to warm up the planet by artificial means if we ever attempted that, by removing more carbon dioxide from the planet, reducing its greenhouse effect, as the planet gets warmer.

Photosynthetic life flourished on Earth at just the right time to cool it down as the sun got hotter, but Mars doesn't need cooling down at present. One way or another, it might not be the most brilliant of ideas to introduce photosynthetic life to Mars, if it doesn't have it yet, until we have a clear idea of what the effect may be.

\section{What about other microbes?}

Most of the papers that study this topic focus on viable life transferred from Mars to Earth. They are generally agreed that this is theoretically possible, though we can't yet prove that it has ever happened. But what about the other direction from Earth to Mars. That direction is central to Zubrin's meteorite argument. This direction is rarely studied, and we get much more cautious statements in the literature in the papers that do mention it.

The big problem with transfer of life from Earth to Mars is the shock of ejection because the material has to leave Earth's surface at very high velocities - not just escape velocity. It has to leave the surface at such a speed that it is still traveling with the escape velocity of 11.2 $\mathrm{km} / \mathrm{second}$ when it leaves the Earth's atmosphere. The smaller fragments especially have to be traveling much faster than that when they leave the Earth's surface. After all, in the other direction, the debris from meteorites typically hits the atmosphere at many kilometers per second, but slows down to terminal velocity, measured in meters per second, before it hits the ground. Imagine how big a rock has to be, and how fast it has to be traveling, to survive ejection from the Earth's surface and still have some of it left to exit the atmosphere at 11.2 kilometers per second!

So the surface of any rock that gets from Earth to Mars will get boiled away, just as for meteorites re-entering our atmosphere and even more so, at least, so long as it has to punch through our atmosphere on the way there. But even more significantly, it's going to 
experience high levels of shock of ejection, to achieve an even higher initial velocity than $11.2 \mathrm{~km} / \mathrm{sec}$, which will damage cells throughout the rock. The best chance for still viable life to get from Earth to Mars is in the early solar system during impacts that were so violent that they blow away part of the Earth's atmosphere, so clearing a gap for the ejecta to travel through on its way into space, without the resistance of the Earth's dense atmosphere. Large impacts also have a larger "spall zone" where the expanding shock wave from the impact meets its reflection (below the surface of the rock) - for details see

We don't get impacts that large any more, so the last chance for something like this to happen is probably more than 3.75 billion years ago. That leaves us with a big question. Was such early life already robust enough back then to survive interplanetary transfer? More recent impacts would indeed send material all the way to Mars. The Chicxulub impactor did just that, 66 million years ago. It would surely have transferred organics, and fossils too. The main question is whether any of the life in that debris was still viable after the shock of ejection from Earth and transit through the Earth's atmosphere. There, there is no way to be certain, but the authors of papers that I studied are often skeptical that it happened in such geologically recent times.

Here is a quote from the conclusion of a 2007 paper (Stöffler et al, 2007).

“'Lithopanspermia' also includes a potential transfer of microorganisms in the opposite direction, i.e., from Earth to Mars. A direct transfer scenario is severely limited because very high ejection velocities in the solid state are required to escape the Earth's gravity field and to pass its dense atmosphere. Favorable transfer conditions may be only achieved by very large impact events, which blow out at least part of the atmosphere. Such impact events happened frequently during the 'early heavy bombardment phase',"

These impacts only happened on early Earth, soon after formation of the Moon. After sterilizing Earth to some depth, they might have reseeded Earth and Mars and maybe other places in the solar system. So if life got off to an early start and was already hardy enough to survive these encounters, Earth and Mars could easily have a common origin for life. Perhaps early Venus, Ceres and other places could also share life with us, or indeed be the source of this life.

We have only limited ground data on the ages of the lunar craters, from Apollo, as they sampled only a small part of its surface, most of it affected by the debris from Mare Imbrium. As a result, most of the earliest cratering history timetable for the Moon, the timescale for the very largest craters, is informed guess work. According to some recent research, such as this paper, perhaps those dates may need to be revised. It's possible that the very largest impacts on the Moon may all date back to as long ago as 4.35 billion years ago or earlier, soon after the formation of the Moon. They think there were no ocean boiling and Earth sterilizing impacts after that. However, there probably were many later large impacts that, though not large enough to boil away Earth's oceans or melt its crust, covered large areas with melts (and could have boiled away the surface of the oceans). See this paper. 
There have been no impacts anything like that for nearly four billion years - Jupiter protects us by breaking up larger comets, diverting them to hit the sun or itself, or ejecting them from the solar system. The asteroids in between us and Jupiter are easily large enough, but they are in stable orbits, and have been for billions of years, and should continue in them for at least hundreds of millions of years into the future (there's a very small fraction of a percent per billion years of Vesta hitting Ceres in the distant future). This is confirmed by the impact crater history on Mars, the Moon, Mercury, what we have of the history of Earth, and the recent few hundred million years,, all that's available, since the last global resurfacing volcanic event, on Venus.

This suggests that for microbes generally, the situation may be similar to the situation for the less shock resistant photosynthetic life. There was a brief window of opportunity for a few hundred million years, during which it may have been somewhat easier for life to be transferred from Earth to Mars (if already hardy enough to survive the journey) during the period of bombardment of Earth by huge impactors that blew away great holes in our atmosphere, and also would have had large "spall zone" where the shock wave meets its reflection so reducing the shock of ejection for the microbes (see More background on whether photosynthetic life could be transferred from Earth to Mars - other photosynthetic species, shock, and spall zone (above). Since then, with the smaller Chicxulub type impacts, the situation is much more uncertain and depended on unusual events.

In short, the transfer from Earth to Mars may have happened billions of years ago. Perhaps it could have happened since then. It's also possible that it never happened.

There is another interesting possibility here. Suppose there was an early transfer but no transfer of any complete microbe for the last three billion years, say.

Though the microbes themselves might not be able to survive transfer from Earth to Mars, their genetic information might be able to get there, as fragments of DNA or RNA. If so, perhaps that genetic information could be taken up by Martian microbes. Perhaps we could find indigenous Martian microbes that incorporate genetic sequences that came from Earth more recently, even as recently as 66 million years ago - even though the microbes themselves did not. This could make us more closely related genetically, through horizontal gene transfer, than you'd expect otherwise for a last common ancestor billions of years ago (McLean et al, 2010)

The bottom line here is that so far we have no confirmed case of life transferred to another planet (panspermia) to base all these ideas on. It is just theory. The direction from Earth to Mars is especially challenging. If there is any life transferred to Mars on meteorites, then surely most life on Earth wouldn't be able to do it.

\section{Accidental similarity of amino acids}

We gave the example of BMAA which resembles I-serine, which can be misincorporated in its place, causing the motor neurone disease, Lous Gehrig's disease. Though such occurrences are rare on Earth, in the case of a biosphere collision there may be many amino 
acids in one biochemistry that resemble but are not exactly the same as amino acids in the other one.

Earth life only uses a selection from the naturally occuring amino acids. Twelve non protein amino acids have been identified in the Murchison meteorite (Kvenvolden et al, 1971), and some may be biochemically active. For instance, isovaline, a commonly occurring amino acid in meteorites, is active as an analgesic, due to an accidental similarity to the amino acids GABA and glycine, inhibitory neurotransmitters in the central nervous system of mammals (Whitehead et al, 2012).

Extraterrestrial biochemistry could use any of thousands of other amino acids. Merringer et al used software to develop a list of nearly 4,000 biologically relevant amino acids that non terrestrial biology could use (Meringer et al, 2013) (Doyle, 2014)

Could motor neuron diseases and other disturbances of nervous systems be the norm after an interplanetary biosphere collision?

\section{Exotoxins, protoxins, allergens and opportunistic infection}

Other issues may arise from secondary metabolites, for instance, Wallemia an airborne extremophile fungus is found in food, especially highly salted or sweetened food such as salted fish, jams and cake. It is adapted to low water activity, and produces the secondary toxic metabolites wallimidione, walleminol and walleminon. W. sebi is a common cause for spoiled food through its production of secondary metabolites. The most toxic of these is wallimidione (Desroches et al, 2014).

Mars conditions are likely to favour life adapted to low water activity levels, and so could be a nuisance particularly for highly salted or sugary foods through production of secondary metabolites, similarly to $w$. sebi.

W. sebi has also been found to cause allergic sensitization (Desroches et al, 2014).

Aspergillus is example of a fungus that acts as an allergen causing asthma in humans. Aspergillus also sometimes acts as an opportunistic infection, and can cause the more serious illness of aspergillosis (Latgé, 1999), and death. Fungi like these kill an estimated 1.5 million people worldwide every year (Brown et al, 2012).

The common allergic reaction to poison ivy is due to Urushiol, a Catichol $\mathrm{C}_{6} \mathrm{H}_{4}(\mathrm{OH})_{2}$ with one or more alkyl chains substituted in the 3 position. It forms antigens by binding to surface proteins of the dermis or epidermis so forming an antigen, which leads to an allergic response on the second exposure (Bryson, 1996, page 680). This again is a simple enough chemical so that it may occur in an alien biology, or something else similar.

Fungi and yeasts are possible on Mars, some are facultative anaerobes, and as we saw, black yeasts are an example of a lifeform that could metabolize in Mars conditions, even 
without any source of water except the humidity in the air, in the close to anaerobic conditions. (Zakharova et al, 2014) The black yeast studied, Exophiala jeanselmel" also occurs as an opportunistic infection in humans (Leber, 2016, page 2240).

For another example, sesquiterpines, is a toxic signaling chemical (semiochemical) produced by potatoes under stress (Matthews et all, 2006). Could semiochemicals produced by an alien biochemistry be accidentally toxic to Earth life.

Alien biochemistries could also produce, or contain protoxins, which when metabolized break down into toxic products. For instance hypoglycin A, which is not itself toxic, is broken down into the highly toxic MCPA-CoA on digestion and can lead to the fatal Jamaican vomiting sickness after eating the unripe fruit of the Ackee tree, a national foodstuff in Jamaica (Holson, 2015). A more commonplace example is methanol which is converted into toxins when digested (Mégarbane, 2005).

Again, perhaps toxicity may be more common if the secondary metabolites or protoxins are based on a different biochemistry.

As before all, if humans are immune, these effects could still harm Earth's biosphere, and harm us indirectly, if other creatures we depend on are affected.

\section{Chemical transformation of habitats by life}

Life can also transform the habitats by chemical modification. In the worst case, this could make most or all the life in that habitat extinct. We have one potential example of this on Earth, in the Great Oxygenation Event, which made its oceans and atmosphere oxygen rich. This process retained many habitats for anaerobes but most of the planet became habitable only to facultative or obligate aerobes.

Some of the original anaerobic microbes might well have evolved in response to the changes and retreated to anaerobic habitats as obligate anaerobes, which we still have today (Lane, 2015). In the sections Is transfer of life possible from Mars to Earth? and Has life from Mars caused mass extinctions on Earth in the past? Was the Great Oxygenation Event such an extinction? we discussed whether martian photosynthesis transferred to Earth could have caused the Great Oxygen Event, and the possibility of a mass extinction, perhaps of earlier multicellular anaerobic life.

It's also possible that we could transfer oxygen producing photosynthetic life to Mars for the first time on our spaceships. Any native life is likely to be adapted to tolerate oxygen because of the superoxygenated conditions. So the effects may not be so dramatic as the Great Oxygenation Event on Earth.

However, if Mars has chemotrophs only, proliferation of photosynthetic life in microhabitats could introduce organics, waste products, toxins, protoxins, etc and so, still change the chemistry of the microhabitats locally. Photosynthetic life could also change the oxygen 
concentration in the brines, outcompete native life, and colonize previously uninhabited habitats on Mars.

As a minor example of transformation, habitats can be transformed through biomineralization. The blueberries found by Opportunity may be an example of biomineralization according to one hypothesis.

The most common form of biomineralization on Earth is the formation of calcium carbonate. There are many biological pathways that bacteria can use to form cement, with some of them used in self healing concrete (Rummel et al, 2017) (Dhami et al, 2013)

One such method may be of special interest to Mars. A consortium of methane oxidising and sulfate reducing bacteria can convert underground aquifers to calcite through anaerobic oxidation of methane (Drake et al, 2015). Perhaps these conditions may occur in the martian subsurface, for instance at the sources of the methane plumes if these originate in geothermally heated underground aquifers.

If there is no native martian life or it hasn't developed the capability, then an introduction of these microbes to Mars could result in the aquifers getting converted to calcites, basically, cement, a suggestion of Conley (Strauss, 2016)

In the backwards direction, if phototrophs in Earth's oceans were replaced by more efficient martian phototrophs phototrophs that happen not to produce oxygen (similarly to our haloarchaea), this could have the opposite effect of the great oxygenation event, and remove the oxygen from the atmosphere over a timescale of thousands of years (residence time of oxygen in the atmosphere).

If life from Mars proliferating in our biosphere produced large amounts of hydrogen sulfide or some other gas which is toxic to humans in minute concentrations, this could also impact on the habitability of Earth for humans without protection. Similarly if it proliferated in water or aquifers and produced toxic chemicals such as perchlorates, again this could impact on us through transformation of the habitats we rely on.

If life from Mars produced significant amounts of methane or other greenhouse gases, in greater quantities than its terrestrial counterparts, this could also impact on Earth's climate through global warming, amplifying warming effects.

\section{Global modification - is a martian Gaia possible and could anti-Gaia lead to a martian swansong biosphere? - NEEDS CITES}

Jack O'Malley-James introduced the idea of swansong biospheres (O'Malley-James et al, 2013) (O'Malley-James et al, 2014) (O'Malley-James 2014). These are biospheres on planets that were once habitable and had abundant life but as the conditions changed, they 
became less and less habitable until life only survives in trace quantities. In such biospheres, life would still linger, but be extremely hard to find and detect.

Consider a future Earth too hot for life, a billion years into the future. It might well have life for a while, living on the edge in a planet that is just barely still habitable. Most, or all of the life there would consist of microbes that can tolerate extremely hot conditions (extremophiles), such as the ones that live at very high temperatures in our hot springs. Eventually even those extremophiles would only survive in a few niches such as caves at the top of high mountains.

As Jack O'Malley-James put it (O'Malley-James 2014, page 36 of his thesis)

"As the limits of life on Earth are explored, the boundary conditions for habitability are pushed further away from the constraints of the traditional $\mathrm{HZ}$. This allows for planets to be considered habitable, but not necessarily Earth-like. Ocean planets, arid planets, ice planets and even rogue planets that have been ejected from their original systems and are traveling through interstellar space can all potentially be considered habitable. For these cases, it is the extremes of life on Earth that hint at the possible biospheres."

Mars may be in a similarly extreme state, Earth-like and very habitable in the early solar system, except, that instead of getting too hot, it got too cold for most forms of life. He makes this comparison in section 6.3 of his thesis.

"Mars could provide a local example of a planet in the very end stages of its habitable lifetime, albeit a planet that has become largely uninhabitable as a result of low temperatures and losing an atmosphere, rather than from runaway heating."

If we introduce Earth life to Mars, effects on its biosphere could be much more extensive than Cassie Conley's example turning methane rich subsurface aquifers to cement.

On Earth photosynthetic life helps make Earth more habitable. It evolved at just the right time to cool down Earth as the sun got warmer, during the Great Oxygenation event. This didn't necessarily evolve as a biosphere response to a warming sun, and may have been accidental.

In the case of Mars, cooling down is the opposite of what is needed. Introducing photosynthetic life would remove carbon dioxide from the atmosphere, already in minimal quantities. It could form an "anti-gaia” on Mars.

Indeed, could this have already happened?

Well, it doesn't actually need life to happen. The Martian atmosphere is remarkably close to the triple point for water at 6.1 millibars average. Perhaps this isn't a coincidence? CO2 is supplied through volcanic outgassing, at a gradually diminishing rate, and the amount of outgassing during the more recent cold dry Hesperian period is thought to have been 
modest. Then there are two main processes involved in carbon dioxide loss, carbon dioxide sputtering and carbonate loss. (Hu et al, 2015).

If the atmosphere increases above the triple point for water as a result of volcanic outgassing, it leads to liquid water forming, either as open lakes, or as transitory pockets of water or moisture, which increases the rate of carbonate formation through carbon dioxide dissolving in the water and that leads to the atmosphere thinning back to 6.1 millibars average_(Kahn 1985) (Nolan, 2008 page 137) (Haberle et al, 2001)

Photosynthetic life would speed up the process. Let's assume that Mars somehow developed carbon fixating photosynthesis or got it somehow from Earth. In that case, photosynthetic life on Mars would set up feedback cycles that limit its own growth, similar to the geological process but on a faster timescale.

This is how it would work:

- Suppose at some point Mars has a thick enough atmosphere for surface water and warm conditions to encourage photosynthetic life

- Photosynthesis removes carbon dioxide from its atmosphere sequestered as subsurface organics. Without plate tectonics then Mars can't return this to the atmosphere again

- This thins its atmosphere and cools it down.

- This makes it less habitable, and reduces the amount of photosynthetic life there.

This feedback loop continues until it is so cold and dry that its photosynthetic life has negligible effect on its atmosphere. At that point then there is still life on Mars, but no longer enough to make a significant difference to the $\mathrm{CO} 2$ levels. Since on geological timescales there is a continuous introduction of some $\mathrm{CO} 2$ into the atmosphere from volcanoes, it would not end up losing all the $\mathrm{CO} 2$ and once the amount of life present is very low the $\mathrm{CO} 2$ builds up again.

The prediction, if this is correct, would be that there may be carbon fixating oxygen producing photosynthetic life there, but if so, it's likely to be hard to detect.

We would expect Mars to have enough $\mathrm{CO}_{2}$ to be barely habitable, but not to have lost so much as to have no life at all. It would occur in such trace amounts that its effect on the atmosphere is only barely detectable, if at all. Indeed, it would look pretty much the same as Mars does to us now, with no life detectable from orbit. There is enough oxygen in the atmosphere to mask any seasonal fluctuations in oxygen produced by life in marginal habitats.

It's also possible that any photosynthetic life is like the Haloarcahea and doesn't produce oxygen. In that case the only effect $\mathrm{n}$ the atmosphere would be to remove $\mathrm{CO} 21$.

Whenever Mars becomes briefly more habitable, if carbon dioxide is released, then the photosynthetic life could then sequester it below the surface. 
The strength of this feedback effect could be amplified by forams in the early lakes and oceans, if Mars developed similar micro-organisms with carbonate shells.

The feedback process involving forams would work like this:

- Some of the dissolved carbon dioxide may get incorporated in the shells of forams so accelerating sequestration of carbonates in lakes and oceans

- This cools down the planet and makes the lakes and oceans less habitable until they freeze over

Once photosynthetic life is no longer active and the forams no longer forming shells, then Mars is free to warm up, and its atmosphere thicken, due to changing eccentricity, or a period of increased volcanic emissions of greenhouse gases, but as it does so, the spread of life across the planet then takes more carbon dioxide from its atmosphere and cools it down again.

The reason there is any carbon dioxide left may be because of the continuing low levels of volcanic emissions on timescales of millions of years.

Another factor would be the infall of organics from comets and meteorites, and, superoxygenated surface layers, and ionizing radiation that dissociates surface organics back to gases such as methane and carbon dioxide and water vapour..

In this way Mars could be locked in a kind of "anti Gaia" feedback loop, which continually keeps it only just marginally habitable. Whenever it warms up and gets a bit more habitable this "anti Gaia" feedback loop would kick in making it less habitable again.

So we suggest that Mars is a swansong biosphere, but in addition, the reason it is in this state may be partly due to an "anti Gaia" effect. In turn, photosynthetic life could be one of the main causes for that. It could be an accident of history - that if photosynthetic life never developed, Mars could have remained more habitable.

This might also explain the trace levels of methane. If there are methanogens on Mars, then as they produce more methane, this warms the planet, leads to more of the photosynthetic life and so leads to $\mathrm{CO} 2$ removed which then cools it down again. So the methane levels remain at trace amounts, because if they increase, this leads to more photosynthesis and $\mathrm{CO} 2$ being removed, and methane as a short lived gas is not able to take its place.

I.e. the Anti-Gaia could be a result of amix of methanogens that warm the planet and photosynthetic life that cools it, but the methanogens are not able to produce enough methane to counteract the effect of the removal of $\mathrm{CO} 2$ by the photosynthetic life form as the amounts of water increase because methane is too reactive to remain long in the atmosphere. 
On the other hand, if it has no photosynthetic life yet, then what we have already is being maintained only abiotically, and photosynthetic life could make the $\mathrm{CO} 2$ sequestration more efficient, cooling it down further and leading to it developing into an anti-Gaia.

If Mars is to develop a positive, warming Gaia then it needs to retain as much carbon dioxide as possible, suggesting an anaerobic ecosystem. Instead of a biosphere based on carbon fixation, it would have a biosphere that uses as inputs the infall of organics from interplanetary dust, comets and meteorites, together with methane emitted from the subsurface perhaps from ancient sequestered clathrates.

Perhaps such a positive Gaia could be created artificially. By careful selection of the members of the biosphere of Mars, such a system would produce methane and hydrogen sulfide. These would replace the negative feedback of carbon fixation with a mild positive feedback leading to increasing habitability as life proliferates (Johnson et al, 2008).

Something like this can also happen abiotically. One hypothesis due to Kite et al for the climate optimum that seems to have happened around 3.7 billion years ago on Mars (at around the time of Gale crater lake) is that it could have been due to release of methane from clathrates. These would come from hydrothermal circulation in the crust in early Mars, formed over a period of up to 100 million years in warm conditions, released in about a thousand years due to sublimation of the ice that traps the methane as the Mars atmosphere collapses. The methane leads to a feedback effect as it releases the carbon dioxide trapped as dry ice, and in turn releasing more methane on a timescale too fast for the ice to form to retrap it, and the result is a climate with typically about 50 mbar of $\mathrm{C} \mathrm{O}_{2}$ but at times of high obliquity over 1 bar with $1-5 \%$ methane.

In this case it is a one off event as the climate optimum lasts for only of the order of 100,000 years, not enough time for the methane to be sequestered as clathrates again. Instead the methane is dissociated photochemically. So, if it happened it is a one-off and can't be sustained.

The authors warn that an exoplanet with a similar signature of methane might well seem to have signs of a life bearing atmosphere in disequilibrium but would be potentially of abiotic origin (Kite et al, 2017).

However, could a similar atmosphere at a lower partial pressure be maintained longer term by some positive gaia effect involving life? The life would need to produce more methane as it becomes more abundant. Indeed if the methane plumes observed on Mars are real and are the result of life processes, for instance feeding on hydrogen released through serpentization in the deep subsurface, then they might be example of such a mild positive gaia effect, though not enough to counteract the photosynthetic anti-gaia.

If Mars has biotic processes reinforcing abiotic processes, that could be an interpretation..

If a Mars like planet could set up a positive Gaia with production of methane based on serpentization as well as organic infall from interplanetary dust, meteorites and comets, it 
would not be so abundant in its production of organics, as Earth's biosphere, but it would still have a productivity that a planet with an anti-Gaia couldn't have.

If a martian gaia could be developed to reinforce habitability, it might encourage $\mathrm{H}_{2} \mathrm{~S}$, which is toxic to humans, or more benignly, methane which is not a toxic gas (except as an asphixiant).

If Mars had an anti Gaia of oxygen producing, carbon fixating photosynthetic life in the past, it would have contributed to build up of organics and carbonates buried below the surface, and because Mars has no continental drift, little of this would ever return to the surface (McKay, 2009).

\section{(ADD CALCULATION OF AMOUNT OF OXYGEN IT WOULD PRODUCE)}

On the other hand, if it has no photosynthetic life then introducing it may make its atmosphere even thinner right now, and make it much harder to restore conditions of a more habitable earlier Mars.

Chris McKay that if we were to find native life there, we might work to make it more hospitable for the native life (McKay, 2009).

If we do this, then we may need to be especially careful not to introduce photosynthetic life, to avoid reinforcing or setting up an anti-Gaia. On the other hand introducing methanogens able to create methane through deep subsurface serpentization or surface processes, or that release $\mathrm{H} 2 \mathrm{~S}$ could help set up a reinforcing positive Gaia, for native Martian life.

We are likely to be at too early a stage in our understanding of ecopoesis to be able to know in advance what the effects would be of an attempt at deliberate creation of a Martian positive Gaia for native life without much more study.

However, it is possible it already has either a positive Gaia or a negative anti Gaia and we suggest this may be an interesting question to motivate analysis of potential ---biological processes on Mars.

\section{Global impacts or minor nuisances in backwards direction to Earth}

In the backwards direction, any of these life forms could be a minor nuisance or have a major global impact on the environment depending on how well the introduced life adapts to Earth's environment. Microbes with a short generation time of a few hours in Earth conditions could also evolve quickly.

If not yet well adapted to Earth conditions, but related to Earth life, even distantly, since the Last Universal Common Ancestor (LUCA) of Earth life, they could also take up capabilities from Earth life via lateral gene transfer. In the other direction, martian microbes could transfer capabilities evolved in Martian conditions of low temperatures, low water activity etc 
to Earth life. This happens rapidly in suitable conditions. In one experiment cited in the ESF sample return study (Amman et al, 2012), gene transfer agents for antibiotic resistance were taken up readily between unrelated spaces of archaea with many of them antibiotic resistant after being left overnight in seawater inculcated with the agents.

An example of a minor but significant nuisance would be a microbe that spoils food at temperatures below $-20^{\circ} \mathrm{C}$ requiring freezers to be operated at lower temperatures, or that spoils jam and salted fish at water activity levels below 0.6 , so requiring jam and salted fish to be kept in freezers.

An example of a major impact would be a microbe that disrupts the basis of an important part of Earth's biosphere, such as a photosynthetic life form that doesn't produce oxygen.

\section{Potential for a more efficient photosynthetic life form based on exobiology - example of a global impact}

Photosynthetic life on Earth hasn't reached anything like the theoretical peak efficiency for photosynthesis. To start with, there's a synthetic $\mathrm{CO}_{2}$-fixing cycle was designed using 17 enzymes from nine distinct organisms including humans which fixes $\mathrm{CO}_{2}$ at $20 \%$ greater efficiency than the Calvin cycle in plants. (Schwander et al, 2016) A 20\% competitive advantage might already be enough to edge out Earth life with otherwise similar capabilities. It can sequester approximately $80 \mathrm{CO}_{2}$ molecules per second (in vitro) compared with RuBisCO, which fixes two to five $\mathrm{CO}_{2}$ molecules per second in plants (Naseem, 2020).

But if martian life uses some radically different principles, it might be far more effective than that. A "mix in a pot chemistry" experiment added cadmium, and the amino acid cysteine (which contains sulfur) to a microbe $M$. thermoacetica that as a natural defense covers itself in nanocrystals of cadmium sulfide, a semiconductor, to protect itself from the cadmium.

This acts as a natural solar cell. The microbe can use this semiconductor to achieve $80 \%$ efficiency in conversion of $\mathrm{CO}_{2}$ to acetic acid. Incoming light converts hydrogen to an electron / proton pair and the proton reduces the amino acid cysteine to cystine. The reaction proceeds for as long as a supply of cysteine is present (Sakimoto et al, 2015). Sakimoto wonders if such a combination could occur naturally with other microbes and other metals (ACS, 2015)

For another example, the Bionic leaf version 2.0 is able to convert $10 \%$ of the sunlight to biomass, using a catalyst made of cobalt and phosphorus with the help of a bacteria Ralstonia eutropha (Biello, 2016). Earth photosynthetic life is only one percent efficient at converting sunlight to biomass.

Also there is no reason for photosynthetic life to produce oxygen. Some consume hydrogen sulfide converting it to sulfur $\left(\mathrm{CO}_{2}+2 \mathrm{H}_{2} \mathrm{~S}=\mathrm{CH}_{2} \mathrm{O}+\mathrm{H}_{2} \mathrm{O}+2 \mathrm{~S}\right.$, the traditional photosynthetic equation with the $\mathrm{O}$ that enters into the reaction replaced by $\mathrm{S}$ ). (Lindquist, 2006) 
More radically,the salt loving haloarchaea convert light directly into energy (through a proton gradient) much as the cells in our retina do, not producing any chemical byproducts at all.

These are not able to fix carbon. However some phytoplankton are mixotrophic, able to both photosynthesize and to consume other plankton (Faure et al, 2019)..

Faure, E., Not, F., Benoiston, A.S., Labadie, K., Bittner, L. and Ayata, S.D., 2019. Mixotrophic protists display contrasted biogeographies in the global ocean. The ISME journal, 13(4), pp.1072-1083.

If a more efficient phototrophic non photosynthetic non oxygenic mixotrophic microbe became the dominant phototroph in our oceans, this could lead to an eventual depletion of oxygen in our atmosphere thousands of years into the future, to the point where humans and most land animals can't breathe the air. It could degrade the environment more rapidly if it is also inedible or poisonous to Earth life or produces hazardous secondary metabolites.

In the forwards direction, with fewer potentially habitable niches on Mars, a relatively small number of polyextremophile species could potentially colonize all or most of the surface habitats.

A minor nuisance would be a microbe that confuses the search for life by co-existing in habitats with the native life, or a microbe that turns the methane generating aquifers on Mars to cement.

The most major impact would be complete extinction of all life that uses the alien biochemistry (as described in the section on Early life above)

\section{Impossibility of quarantine provisions until you can assess the risk}

The idea of quarantining returning astronauts, as used for Apollo, was never subject to peer review. In particular, would a two week quarantine guarantee that they are free of diseases? Carl Sagan gave the example of leprosy which may often be symptom free for decades.

Then you have to consider, how would NASA or any other space organization respond in the case where an astronaut in quarantine does get sick? Should hospital treatment be refused because it's not known if this is a disease caused by Martian life or an unusual Earth based disease? It also raises issues of human rights.

Can an astronaut be held to a prior commitment to stay in quarantine facilities when it's not possible to prove that it would be hazardous to Earth to leave them?

Margaret Race mentioned that similar legal issues apply to a robotic sample return. What is the status of a worker inadvertently exposed to the sample? Should they also be kept in 
quarantine indefinitely, until their release is shown to be safe for the environment of Earth, and if so, is that consistent with human rights? (Race, 1996)

However this issue with quarantining astronauts gets far more acute when considering a wider range of microbial life than human diseases alone.

Astronauts could be passive carriers of microbes, although symptom free on Mars, and completely free of any microbes that can cause disease in humans.

They could return microbes to Earth in their spaceship, in their clothes, the air, or in and on their bodies that may lead to anything from a minor nuisance to a major impact on our ecosystems.

To take a concrete example, suppose that they return mirror life to Earth. This might not show up any issues in a habitat on Mars or during the journey home. The microbes could also evolve and adapt in Earth conditions after return here and at some point spread widely.

Related martian life could also share capabilities with Earth microbes by parallel gene transfer, either gaining the abilities from Earth life to survive here, or conferring new ability to terrestrial life.

The conclusion seems inevitable, that quarantine is not able to protect from backwards contamination in the initial phases, when you do not yet know what it is you need to quarantine against. There is no substitute for understanding what is present there first.

Quarantine procedures could be useful at a later stage. Once we develop an understanding of the martian biochemistry and have knowledge of particular hazards that need to be contained we might find that quarantine works for some of them.

Other potential biohazards in the backwards direction could not be quarantined against in any way since they produce no harmful effects in humans. An example might be spores of phototrophs that risk causing degradation of Earth's biosphere if returned to Earth. If a spore of a phototroph phototroph can remain viable for millions of years, no quarantine period could be long enough.

If biohazards like this exist on Mars, Europa, Enceladus or elsewhere, they would have to be prevented from entry to Earth's biosphere by sterilization and / or $100 \%$ containment. Those are measures that can't be applied to astronauts. 


\section{Legal and practical issues for sample returns - a likely minimum of 20 years from initiation of legal procedures to permission for launch of the sample return mission}

Each review of Mars sample return mission protocols has added new requirements.

Originally the requirement was a simple gas-tight glove box in a biocontainment level 4 facility. (Board, 2002, page $\underline{\text { 11) }}$

Requirements got more stringent in two subsequent studies by the NRC (NRC, 2009) and then by the ESF (Amman et al, 2012), with the discovery of ultramicrobacteria and then the discovery that archaea can readily transfer capabilities overnight in lateral gene transfer via GTAs, and the work on the limitations of size of early life which produced theoretical models of micro-organisms that may be able to replicate with cells only tens of nanometers in diameter, requiring containment of particles at well below the resolution limits of a diffraction limited optical microscope.

Currently the recommendations are for a $\$ 500$ million facility with design requirements never fulfilled before. The 2012 study by the ESF left open the possibility that future studies may increase the requirements further.

The facility also has to be operational and in use at least two years before the mission to collect the sample is launched from Earth. Preliminary studies have warned that it may take as many as 7 to 10 years to get it operational, followed by an estimated 5-6 years to become familiar with the procedures (NRC, 2009)

But the legal situation would have to be sorted out too, before the mission could be launched, and most likely the facility would not be built until the legal provisions are in place.

Margaret Race looked in detail at the legal processes that would have to be completed before we can return a sample from Mars to Earth, even to a purpose built receiving facility (Race, 1996).

Before a sample return, we have to accomplish, in this order

- Several years: Formal environment impact statement for NEPA + laws on quarantine to be enacted, involving broad public consultation. The average length of time for an EIS in the twelve months ending 30th September 2016 was 46 months (DOE, 2016).

- Several years: Presidential review of potential large scale effects on the environment, after other domestic laws.

- Can be done alongside the other work: International treaties to be negotiated and domestic laws of other countries 
Margaret Race does not estimate a total time for all this. As a rough estimate, a decade would seem optimistic to complete it all.

- 7 - 10 years: building the facility (NRC, 2009)

- 2 years: operational before launch (minimum requirement, more likely 5-6 years) (NRC, 2009)

- 2 - 4 years: to collect the sample and return it

(if we follow the Mars 2020 plan then the follow up rover has to retrace at least part of the path of Mars 2020 to retrieve the samples, in tubes that are dropped on the surface of Mars in small caches from time to time).

Total 11 - 20 years.

If everything went without a hitch, it would be optimistic to aim for a sample return mission that launches less than 20 years after the legal process starts. The process of building and testing the facility alone could take 20 years.

This means that if we want a sample returned to Earth by 2040 we should start on this process right away in 2018 - there is no time to lose. Indeed, it is probably already too late to achieve a launch date of 2040. It would be no great surprise if it was delayed to 2050 .

What makes the legal process so complex, and the facility so expensive is that we have to prove that the facility and the sample return provisions can protect Earth from any conceivable alien biochemistry when the only biochemistry we know of is on Earth. We would build the same facility to receive an unsterilized sample from a habitat in the Proxima Centauri system!

Over a period of two decades it takes to pass all the laws, then approve the facility, build it and test it, our understanding of Mars through in situ searches could develop to the point where we prove that native Mars life is harmless and discover that the building is unnecessary.

Yet the lessons learnt from Apollo show that we have to take great care, if our backward contamination measures are to be more than a token measure. If we are to take protection of Earth seriously we have to do far better than they did (Meltzer, 2012). Many examples of environmental issues have led to a situation where protecting the environment of Earth is considered to be of utmost importance to humans. If this is accepted, then it is right that such a procedure should have detailed impact assessments, and that public involvement is encouraged at every stage of the process.

So, what can we do? Some sample returns are unproblematical. We can return samples from asteroids or comets, based on the "Natural contamination principle" (Greenberg et al, 2001). Earth is constantly hit by samples from such objects anyway. The Moon has also been passed for unrestricted sample return.

However the conclusion seems inescapable for situations where we can't demonstrate natural contamination processes like that. Legally, as a result of both domestic and 
international laws, we can't return an unsterilized sample from Mars, Europa or Enceladus, before 2040 if we start on the legal proceedings right away. The process might well take several more decades to complete, especially if there are objections at any stage.

Perhaps there is an easier way to handle this? All the legal and practical issues arise from the requirement to return the sample to Earth's biosphere for study. Suppose we return them to some other location close to Earth?

\title{
A method for $100 \%$ safety for sample returns without the legal complexity or the issues of building the receiving facility
}

\author{
Carl Sagan wrote in his book Cosmic Connection (Sagan, 1973):
}

...The likelihood that such pathogens exist is probably small, but we cannot take even a small risk with a billion lives

Following the same approach as for the forward direction, I suggest we should respond to these issues by being more, rather than less ambitious, and shouldn't set our sights any lower than $100 \%$ safety for sample returns, following Carl Sagan's suggestion that we shouldn't take even a small risk with a billion lives.

This is not an impossibly idealistic goal. Indeed, there are at least two ways to achieve this. Either study the life in situ first until it is thoroughly understood, and devise precautions accordingly - or else - return it to a location where contamination of Earth is impossible.

The natural place to do this may seem to be the moon. However, this would constitute a possible contamination of the moon by martian life. The problem is that in the near future humans are likely to return to the Moon. With the prospect of frequent travel back and forth, this means that the Moon also has to be kept free from backward contamination from Mars and further afield, to protect humans on the Moon, and also the Earth from materials that may have got there as a result of the sample returned to the Moon.

The COSPAR guidelines for category 5 (sample return) missions currently say that (COSPAR, 2011)

"Category $\boldsymbol{V}$ missions comprise all Earth-return missions. The concern for these missions is the protection of the terrestrial system, the Earth and the Moon. (The Moon must be protected from back contamination to retain freedom from planetary protection requirements on Earth-Moon travel.)"

Arguably, it's possible to keep a facility in a remote area of the Moon biologically isolated from the rest of the surface. Perhaps this could be a solution. It would be necessary to consider levitation of the lunar dust, especially after rocket landings, and it would also involve contingency planning for sample return missions that crash on the Moon, but it seems not impossible. It would be far easier to modify the COSPAR guidelines for the Moon than to introduce the legislation needed to permit return to Earth. 
However a simpler approach would be to return samples to a few thousand miles above GEO. This is an orbit that is as far as possible from both Moon and Earth in terms of delta va as possible - more than one kilometer / second in both directions.

Satellites in Geo have low relative velocities, all orbiting in the same direction around Earth in geostationary orbits. So, It is far enough from GEO and also from its graveyard orbit, even if satellites there are serviced by humans in situ. It's also close enough to Earth to make it easy to send instruments to the facility to examine the samples telerobotically, especially with future heavy lift.

It is also a feasible orbit to return samples too. This can be done by first returning them to a distant retrograde orbit around the Moon as suggested for the now discontinued Asteroid Redirect project, without need for risky aerobraking in the Earth's atmosphere (Strange et al, 2013) (Pires et al, 2020).

The facility could start out as a single spacecraft to receive the sample. It would have tools to do preliminary analysis, and sterilize sections of it to send back to Earth. Once these are examined, if it is proven to have no life, the whole thing can be sterilized using ionizing radiation to make sure there is no life in it, at similar sterilization levels to Mars meteorites that have spent a few million years in transit, and returned to Earth. This would not impact on the geological or geochemical interest of the sample, and in the remote case that there is life in the sample, not detected in the preliminary analysis, it would still be recognizable as such..

If there is interesting astrobiology in the sample, then, depending on its characteristics and whether it is thought to have potential to impact on the environment of Earth, then it can be studied in situ as part of a growing telerobotic facility. This can be done until whatever time it is proven that it is not hazardous to Earth's biosphere.

This would

- Obviate the need for a decades long process of passing laws sequentially to permit return of a sample to the Earth's surface.

- Remove need for an upfront cost to build the receiving facility. Instead the telerobotic facility in orbit is only developed after the sample is returned, if study of the sample shows that it is required

- Fully protect the sample from Earth's biosphere, and Earth from the sample. The 40,000 miles of vacuum between the facility and Earth provides more than adequate protection.

If eventually we do return it, after telerobotic study, it would be in a situation where we know what is in the sample, and what it's capabilities are, and can then decide what precautionary measures are needed based on what we have. To take an extreme example, if it is an early form of life, based on RNA, imperfectly replicating with metal catalysts and requiring agitation to replicate like the RNA only protocells currently being researched in the Szostack 
lab (Szostack, 2016), then we'd probably be able to decide early on that there is no risk to Earth life.

In those circumstances, the sample return could then be handled through COSPAR similarly to a sample return from a comet or asteroid, and there would likely be no need to go through any more of the legal process.

While if it is mirror life or some other form of life that could replace terrestrial microbes in our ecosystems, or a phototroph that's more efficient at photosynthesis than any Earth based life, or it has a metabolism that would not be recognized by the immune systems of Earth life, or it is a disease of biofilms that could invade lungs similarly to Legionnaires disease, or it produces toxins, or exotoxins or its metabolism itself is poisonous to Earth life, or it is capable of replicating at temperatures below those of our freezers, etc, we'd need to take appropriate measures or continue to study it in situ in orbit using telerobotics.

In the case where the decision is made that it is potentially hazardous to Earth's environment, new legislation would be needed to return it. However, it would be in a context of dealing with a known danger with known containment requirements. The process would be simplified, and there would also be less risk of a lapse of containment procedures since the scientists and technicians handling it would already know it is high risk.

In this way we never need to jeopardise the safety of either our astronauts or Earth itself. We can also reduce upfront cost and legal complexity and build only what is needed, rather than build a multi-purpose facility to return all samples with any conceivable astrobiology. At each stage the process is based on knowledge rather than attempts at extrapolating the capabilities of an unknown biochemistry from our understanding of Earth life.

\section{Assessing the risk - Drake type equation approach}

Astrobiologists, when asked to assess the chance of degradation of Earth's environment or other hazards for studies of a Mars sample return, generally say words along the lines of "the chance is probably low, but we can't say that it is zero". But even this much is really asking far too much of them. With no experience at all of any biosphere collisions, how can we guess any probabilities here? Might it be best just to guess 50 / 50 that it could be harmful, not knowing anything about it?

I will present a Drake equation approach that may help with thinking about the probabilities of damaging biosphere collisions. Although it can't answer the question, yet, by putting various numbers into the equation, it may help clarify thought on this topic, similarly to the Drake equation.

Let's define probabilities as follows, where each one is conditional on the previous one: 
$>p_{g}$ that life ever evolved on Mars or got transferred to Mars from elsewhere via panspermia

$>\mathrm{p}_{\mathrm{s}}$ that if life was there in the past that it survives to the present

$>\mathrm{p}_{\mathrm{a}}$ that the surviving life includes advanced life as capable as Earth life or more so

$>\mathrm{p}_{\mathrm{d}}$ that this advanced life is capable of degrading the environment of Earth.

Then, the chance of severe degradation of the environment of Earth after unsterilized uncontained sample return is $p_{g} \times p_{s} \times p_{a} \times p_{d}$

Setting all these numbers to $1 / 2$ gives us $1 / 16$ for the chance that what we encounter on Mars would cause severe degradation of the environment of Earth if returned unsterilized without containment.

The equation doesn't rule out the possibility of multiple independent evolution of life on Mars, or that some Earth life has got transferred to Mars in the past. Also life could have been made extinct many times and re-evolved, or it could have shadow biospheres, even multiple simultaneous types of biochemistry, or a mix of habited and uninhabited habitats (Cockell, 2014).

The idea with this equation is to keep it simple. If wished, one could devise more complex equations to cover more elaborate ways in which life could have survived to the present and what form it could take,

The main point here is that it's not really fair to ask this of astrobiologists, to try to come up with a probability based on zero previous experience of other biospheres. But this may help focus ideas about possibilities, and though it can't give a definite answer, and nothing can, it could be instructive to look at the range of numbers resulting from differing approaches and assumptions.

\section{Mathematics of backwards contamination}

In the backwards direction, the planetary protection requirements for a Mars sample return facility are typically based on the idea of a "one in a million" chance of part of the sample escaping containment as the "gold standard". This is not based on any calculation, it is just a figure that is traditionally used for biosafety facilities. But in this case, if the backwards contamination risks degradation of the environment of Earth, is a one in a million risk low enough?

Some colonization enthusiasts are so keen to colonize Mars that they might answer without hesitation that 
"Yes, of course we shouldn't hesitate from taking a risk as small as one in a million of degradation of Earth's environment for something of such value as setting up a colony on another planet".

If you are like Carl Sagan who thought we shouldn't take even a small risk with a billion lives you may find that puzzling. However, it is understandable if you read that one chance in a million as a personal risk.

We run far higher risks than that in our personal lives. For instance the lifetime chance of dying in a motor vehicle crash in the US is 1 in 112 (NSC, 2017), and compared to that this back contamination risk is indeed negligible on a personal level.

The difference is that this time, rather than a personal risk, it is a risk taken by someone else that in the worst case can impact on the whole of humanity.

Even for a minor nuisance, how many would say it is worth taking a one in a million chance that from now on, after the sample return, that we have to buy new freezers that operate at $-70{ }^{\circ} \mathrm{C}$ instead of $-20{ }^{\circ} \mathrm{C}$ because returned Mars microbes make food mouldy even kept at $-20{ }^{\circ} \mathrm{C}$ ? Or that honey, jam, cake and salted fish have to be kept in fridges? When it becomes an action that could become as much as a minor nuisance in their lives, people may think differently about such matters.

When it comes to a major impact on the environment of Earth, many would agree with Carl Sagan that we can't take even a small risk with a billion lives.

For instance, is it okay to take a one in a million risk that one or other of these things happens:

- Many people develop a severe motor neurone disease leading them to be confined to wheelchairs, have shorter lives, and eventually only able to speak with a speech synthesizer, as a result of the sample return leading to life proliferating that uses BMAA in place of I-serine - and from then on humans can only avoid this disease if they eat food that's grown in special greenhouse enclosures designed to keep out the martian life

- A returned photosynthetic life form takes over from the oceans leading to eventual depletion of oxygen in the atmosphere a thousand years from now and need for humans to live in enclosed habitats with photosynthetic life or artificially supplied oxygen starting from a few centuries from now

- Or some other major impact such as some of the ones explored in this article

There's an interesting way of working with this mathematically derived by Nick Bostrom which may help understand the perspective of those who think a one in a million chance of a severe impact like this is unacceptable. His approach is to multiply the probability by the population to get the expected number impacted (Bostrom, 2002). 
Let's apply his approach to this back contamination scenario. With a population of 7.5 billion and a 1 in a million chance of a severe impact, suppose that it affects half the population, then that multiplies out to an expected 3,250 people that would be impacted by the sample return.

But if it is something that has long term future effects on our ecosystem, leaving Earth significantly less habitable to humans for all future time - then the numbers become far greater. For instance, if you look forward a 100,000 years, or 3,000 generations, those 3,250 people become 9.75 million.

Nick Bostrom suggests that this can give a way to think about these existential risks, that take us out of our instinctual responses.

As he explains in his paper, it becomes even more of an impact if you consider a possibility of human extinction. The potential numbers of future humans that could be impacted by extinction (can no longer come into existence) in our solar system alone may number in the trillions. The asteroid belt has resources sufficient to make habitats spinning for artificial gravity (not spinning hollowed out asteroids, but rather, the asteroid materials made into habitats) with total living area equal to a thousand times that of the land area of Earth. Future populations could easily amount to trillions, or even more if they expand out to the resource rich icy bodies of the outer solar system and then the Kuiper belt and beyond using large thin film mirrors to concentrate the sunlight.

However, l'd argue that total extinction of humans is unlikely in the case of back contamination. One way or another it should be possible to keep out the alien microbes from enclosed habitats designed like space habitats even in the worst case scenarios. They would be far easier to construct on Earth with equal pressure inside and out, and sterilization procedures to prevent a by then known and well characterized exobiology from entering the habitats.

That leaves us with those expected numbers of millions of future people dying or severely impacted if there is as much as a 1 in a million chance of severe degradation of the environment of Earth after a breach of sample return.

There is no way to argue this case in a logically conclusive way, without going into areas of ethics where agreement is unlikely. However perhaps this can help some of the Mars colonization enthusiasts to appreciate the point of view of those who, like Carl Sagan, advocate a more cautious approach for sample return.

Sample return studies have so far used a value of 1 in a million chance of contamination as acceptable. But I would submit that considered in this way, the number should be far lower, at least for any sample that risks degradation of the environment of Earth, as described in the Outer Space Treaty text. Or best, we should not run a small risk but have a method that $100 \%$ guarantees that Earth is safe from back contamination until we know what it is that we could return from Mars. 


\section{Drake type equation approach to extinctions in the forward direction}

When it comes to collisions of entire biospheres, it's possible that our imaginations are not able to prepare us for what we may find. We have had no previous experience of such a collision. At any rate we have to think beyond the limitations of what happens with Earth analogue environments.

Again the Drake type equation may help. With each probability conditional on the previous one, let's define

$>\mathrm{p}_{\mathrm{g}}$ that life ever evolved on Mars or got transferred to Mars from elsewhere via panspermia

$>\mathrm{p}_{\mathrm{s}}$ that if there was life on Mars in the past, that it survives to the present

$>\mathrm{P}_{\mathrm{e}}$ that surviving life includes early life either as the only form of life or as a shadow biosphere

$>\mathrm{p}_{\mathrm{t}}$ that this early life is made extinct in its totality after it encounters modern Earth life.

The chance that forward contamination means that we lose the opportunity to study prebiotic chemistry in a world that has never had life would be just

$>1-p_{g}=$ probability that what is there is pre-biotic chemistry that never developed life

We'd have a chance of

$>1-p_{g} \times p_{s}=$ probability that what is there is pre-biotic chemistry that never evolved to life or that developed life but then lost it.

With this approach of just assuming a $50 \%$ chance for $\mathrm{p}_{\mathrm{g}}$ and $\mathrm{p}_{\mathrm{S}}$, then that would be a $50 \%$ chance that with forward contamination we lose the opportunity to study prebiotic chemistry on Mars.and an additional $25 \%$ chance that we lose the opportunity to study uninhabited habitats with non biotic chemistry on a previously habited world.

The chance that all Mars life is eventually made extinct after irreversible forward contamination is

$$
p_{g} \times p_{s} \times p_{e} \times p_{t}
$$


Setting all these numbers to $1 / 2$ gives us $1 / 16$ for the chance that what we encounter on Mars is made extinct in its entirety by Earth microbes. Where, once more, as for the Drake equation, readers can try other numbers in place of $1 / 2$ for each of those possibilities.

In both cases, Earth microbes might rapidly spread to all habitats in the vicinity of a human landing, after the first dust storm. It might take a fair while to recognize what it is we had lost.

\section{New version of the precautionary principle for superpositive outcomes}

The precautionary principle was developed to help deal with some of the new unprecedented challenges faced by humans.

"We believe there is compelling evidence that damage to humans and the worldwide environment is of such magnitude and seriousness that new principles for conducting human activities are necessary.

"While we realize that human activities may involve hazards, people must proceed more carefully than has been the case in recent history. Corporations, government entities, organizations, communities, scientists and other individuals must adopt a precautionary approach to all human endeavors.

"Therefore, it is necessary to implement the Precautionary Principle: When an activity raises threats of harm to human health or the environment, precautionary measures should be taken even if some cause and effect relationships are not fully established scientifically.

"In this context the proponent of an activity, rather than the public, should bear the burden of proof.

"The process of applying the Precautionary Principle must be open, informed and democratic and must include potentially affected parties. It must also involve an examination of the full range of alternatives, including no action."

\section{(Raffensperger, 1998)}

This applies in the backwards direction, to a sample return, if we decide to return a sample to Earth. The affected parties there are everyone on the Earth because it could, potentially, impact on the habitability of the Earth.

The main point there is that it has to involve examination of the full range of alternatives including no action. 
It's not so clear though in the forward direction. If we contaminate Mars or Europa or Enceladus with Earth life, there is no risk to human health or the environment, at least, not the environment of Earth, so we can't motivate this in the same way as the precautionary principle, by looking at the negative effects.

However, we can also have a mirror version this, what we might call a "super-positive outcome". There might well be discoveries to be made in the field of biology, such as XNA life or using it as a window into the stages between life and non life - that we could only find out otherwise by travelling interstellar distances (and maybe not even then).

We don't have a risk of immense harm, but what we do have is a risk of destroying a potential future benefit of immense value. This suggests the need for a positive version of the precautionary principle. It's based on the idea that with the technology we now have, we may meet situations where we can see a prospect of a potential for a vast future benefit, but we can't prove that this benefit will occur. We could call such an outcome a "super positive outcome".

Proposed definition: a "super positive outcome" is one which has positive transformative effects on us, our children and all future generations and civilizations. For example, discovering some alternative form of life or early life on Mars could revolutionize biology, could potentially benefit medicine, agriculture, and indeed anything that we do that uses products of life, also nanotechnology. It could potentially, in the best case scenario be a hugely positive transformative discovery.

The principle then would cover situations where we have a potential, but not proven, future positive outcome, and actions that can undermine that outcome.

As with the precautionary principle, there may be no way we can establish the cause and effect relationships thoroughly before it happens. Until we can do a biological survey of Mars, there is no way to prove that Earth microbes could make Mars life extinct, or to prove the other way that they will cause no harm. To do that we need to find out whether Mars has habitats for Earth life, whether those habitats contain indigenous Mars life, and whether Earth microbes can make the Mars life extinct. We also have no way to decide how much of a benefit our scientific knowledge of it can be.

So, I suggest we should have similar guidelines to the precautionary principle for super positive outcomes - outcomes of potentially overwhelming positive value for humanity. So, let's try just replacing "threats of harm to human health or the environment" by "super positive outcome" and rephrase the rest of it accordingly in a positive way. We get something like this:

"When an activity impacts on a potential super positive outcome that may be of overwhelming positive and transformative value for humanity, precautionary measures should be taken even if some cause and effect relationships are not fully established scientifically. 
"In this context the proponent of an activity, rather than the public, should bear the burden of proof.

"The process of applying the precautionary Principle for superpositive outcomes must be open, informed and democratic and must include potentially affected parties. It must also involve an examination of the full range of alternatives, including no action."

We can still do things that impact on extraterrestrial life. The principle is saying, however, that if we do, then as with the precautionary principle, it has to be on the basis of knowing clearly the consequences of our actions. Also it can't be a decision of a few enthusiasts making on the spot executive decisions. When there is a super positive outcome involved, we need public debate and open and informed democratic discussion of whether we should do it. As with the precautionary principle in the backwards direction, "no action" always has to be on the table as an option.

Also, as for the precautionary principle, when we don't know the effect of our actions, the burden of proof has to be on the proponent for the activity, such as to land humans on Mars. They would be responsible for providing the evidence we need to assess whether it is going to impact on a super positive outcome. If they can't do that, then it's reasonable to ask for "no action" until we understand the situation better.

We can then use a positive version of Nick Bostrom's approach. For instance, a 1 in 10,000 increase in the probability of a super positive outcome for a billion people, due to more care taken over planetary protection measures, means that the expected number of people who benefit is a hundred thousand.

It's well worth going to a fair bit of effort to do something that has an expectation of a positive benefit to a hundred thousand people. My new suggestion is that it is worth doing that, even if it is a tiny probability of a super-positive outcome that could benefit billions of people.

Then, as with Nick Bostrom's argument, if you take account of future generations as well - all the future generations that would be able to study your contamination free Enceladus ocean - then potentially trillions, and even quadrillions or more people may be affected by a decision about whether or not we take a 1 in 10,000 risk of contaminating the Enceladus ocean right now.

\section{Mundane value of astrobiology for business - example of the billion dollar extremophile enzymes industry}

I think many would agree that it is important not to extinguish native life on Mars for astrobiology, because of a shared interest in learning about our origins, and about whether life is possible around other planets and other stars, and what such life might be like. 
However many may not realize that it can also be important in more mundane ways too. For instance study of extremophiles has lead to enzymes that are widely used (Sarmiento et al, 2015)

- in the $\$ 1$ billion industry of enzymes for detergents - this is another application - they work at cold temperatures so removing the need for heating and saving energy.

- in the food industry, including bread making, fruit juices, for lactose free foods, for making syrups for wood pulp and paper processing

- in the textile, cement, cosmetic industries.

- in various research techniques for experts studying DNA and RNA

They are used to reduce costs, make the processes more eco friendly, reduce $\mathrm{CO}_{2}$ emissions, enable more efficient faster processing, etc etc.

The cold adapted enzymes are more active, so less of the enzyme is needed, and they can be used at lower temperatures, saving energy. The heat adapted enzymes are active and efficient at high temperatures, extreme $\mathrm{pH}$ values, high concentrations of the substrate, and high pressures. They are also highly resistant to organic solvents, and other things that stop enzymes working (denaturing agents). They are easier to separate during purification steps (because they don't break up) and they catalyze faster reactions.

Discoveries from study of a novel biochemistry could be far more radical than these enzymes, with implications for pharmaceuticals, understanding processes in medicine, agriculture, nanotechnology, and many other fields. They may form materials with novel properties too (many materials we use in everyday life are the results of biology).

Indeed, it's impossible to know what the results would be. These lifeforms if they exist, and any discoveries that would flow from them, are part of our natural heritage as human beings living in our solar system.

Is it right to take a $1 / 16$ risk of losing this heritage - or whatever number we come to when we plug in our figures into the equation?

If the risk could be reduced to as low as 1 in 10,000 - would that then be acceptable as a risk of forward contamination? This 1 in 10,000 figure is one that is used in planetary protection discussions as an acceptable per mission risk for forward contamination. Although it is no longer a requirement to calculate probabilities as it was for Viking, the motivation is still a 1 in 10,000 probability, for historical reasons.

The origin of this figure is unclear. It may be from an COSPAR resolution from 1964 which used this figure, but not as a probability of contamination, rather, as the probability of there being a single viable microbe present on the spacecraft, a far stricter requirement (Young et al, 1965). It may alternatively come from a chapter written by Carl Sagan and Sidney Coleman, published in 1966 and reporting on a study carried out from 1964_(Sagan et al, 1966). In that case it was a figure he used to illustrate a method of calculating a per mission probability to achieve a desired overall probability of contamination of Mars over a fixed exploration phase of 1 in 1000 . 
The participants in the COSPAR workshop in 2000 on preventing forward contamination of Europa agreed that there is no justification possible other than historical precedent for any particular figure over any other figure, but decided to go with this customary figure on the basis that they had no reason to change it to any other number (Board et al, 2000).

If you transfer such a probability to some other area of life where people handle items of great value, suppose an antique dealer has a track record of accidentally dropping 1 in 10,000 of the items they handle. Would you give such a dealer a precious Ming vase to appraise it? Should we be less careful about such a unique heritage and birthright as early life on Mars, or in the Europan or Enceladus oceans as we are about a Ming vase?

Our near future decisions may become more important than we realized. If the potential effect of Earth microbes on Mars, Europa or Enceladus is to make native Mars microbes extinct, or to erase traces of prebiotic chemistry that we could have studied in situ on a pristine planet, the stakes for successful planetary protection may become high indeed.

As the participants of the 2000 workshop commented, there seems no particular reason to choose any other figure in place of that 1 in 10,000. But what if we can achieve $100 \%$ certainty of no forward contamination?

\section{Are $100 \%$ sterile rovers and instruments possible in the forward direction?}

(work in progress)

Dry heat sterilization (actually with a small amount of humidity to help decompose the organics, despite the name) is the most established method for planetary protection, used since Viking. The other methods that are approved by NASA and ESA are gamma radiation, and low pressure hydrogen peroxide. There are many other ideas under investigation (Pugel et al, 2017).

However none of these currently have the design goal of $100 \%$ sterilization. That design goal is feasible if we can find some process that electronics can withstand and life can't.

Heat sterilization seems the most promising. No Earth life can survive temperatures of hundreds of degrees centigrade. At high enough temperatures, organics get decomposed as well, and not even gene transfer agents, RNA or proteins will survive.

The main issue with heat sterilizing modern spacecraft is that many modern instruments can't tolerate these high temperatures either. They can be damaged or go out of alignment, Solders and resins may melt, monolayers can evaporate, and even the sterilization temperature for Viking of $112{ }^{\circ} \mathrm{C}$ for 40 hours is too much for many modern electronic components.

However this is only because normal consumer electronics do not need to be designed to withstand high temperatures. They can be built at a lower cost with components that are not 
heat tolerant. Our capability to design components that function at high temperatures has improved significantly since Viking, but is only used for components that need this capability.

So far, this idea of high temperature tolerant spacecraft has been explored mainly for Venus surface missions. This is for ideas of a rover to operate at Venus surface temperatures for long periods of time. One option is to cool it to $200-300{ }^{\circ} \mathrm{C}$. Another is to design it to run at $500{ }^{\circ} \mathrm{C}$ with no cooling. NASA are exploring both options

The lower temperature range of $200-300{ }^{\circ} \mathrm{C}$ is easiest to design for. Many commercial components including microprocessors and memory devices, already function at these temperatures. They are used in oil wells, aviation and electric cars.

The temperature of an oil well goes up by $25^{\circ} \mathrm{C}$ for each kilometer of depth. Now that shallower oil fields are exhausted, oil is extracted from "hostile wells" with temperatures in excess of $200^{\circ} \mathrm{C}$ and pressures in excess of 25,000 psi (over 1,700 atmospheres). Cooling is not practical in those conditions so they need electronics that can work at those temperatures.

High temperature electronics also save weight on planes. They don't have to be cooled, and they can be placed closer to the engines. This helps with cost, weight and most important, reliability (there are no worries about what happens if the cooling system fails). Electric cars also can be more reliable, weigh less and cost less if the electronics can be placed right next to the engine components at high temperatures. High-Temperature Electronics

High temperature mechanical components, sensors and cameras are all possible with the correct choice of components. The batteries and solar panels are best replaced by RTG's (Radioisotope Thermal Generators) which can withstand high temperatures. Radio communication can be done with high temperature components and high temperature mechanical components are also possible. All these ideas have been explored in the Venus rover design studies.

If we adopt this approach then future instruments for Mars special regions and subsurface oceans of icy moons and dwarf planets will need to use chips, solders and other components that work up to high enough temperatures for $100 \%$ sterilization.

Since these temperatures are only used for sterilization, unlike the situation for a Venus rover, the instruments do not have to operate at $200-300{ }^{\circ} \mathrm{C}$. They just have to be able to survive heating at that temperature for several months on the journey to Mars. Once there, they would operate at normal temperatures.

In the system of planetary protection used for Curiosity and our other modern rovers, the computer section has to be isolated from the rest of the spacecraft with filters to keep the microbes inside it. This is no longer needed if the electronics is certified to operate at $200{ }^{\circ} \mathrm{C}$ or $500{ }^{\circ} \mathrm{C}$. The whole spacecraft can be sterilized, as for Viking, but at a far higher temperature using modern more heat resistant electronics. 
The simplest way to do this might be to use the spacecraft to heat itself. If it uses an RTG as a source for power, it can also be used as a heat source too. Typically an RTG has an active cooling system or heat radiators. If this is disconnected, with the insulation of the vacuum of space, the RTG could heat the entire spacecraft during the journey out.

The lowest temperature of $200^{\circ} \mathrm{C}$ would sterilize it of all life during a six months cruise.

This might still permit forwards contamination by RNA, proteins, etc. This could be significant, especially if it might explore prebiotic chemistry or early life. Also, GTAs could transfer properties of Earth microbes via lateral gene transfer to life related to modern DNA based Earth life.

However, at $250{ }^{\circ} \mathrm{C}$ the half life of the RNA bases under hydrolysis is between 1 and 35 minutes, with $U$ the most stable, $\mathrm{G}$ and $\mathrm{A}$ of intermediate stability and $\mathrm{C}$ decomposing most rapidly. This suggests that if it is heated for several months at $250{ }^{\circ} \mathrm{C}$, so long as some water vapour is available for hydrolysis of the bases, then there is not likely to be any genetic material remaining by the time it reaches Mars. If the temperature can be raised to $350{ }^{\circ} \mathrm{C}$ then the half-lives are between 2 and 15 seconds (Levy et al, 1998).

$300{ }^{\circ} \mathrm{C}$ should be enough to destroy proteins too. Eight of the 20 amino acids, G, C, D, N, E, $\mathrm{Q}, \mathrm{R}$ and $\mathrm{H}$, have been proven to not just evaporate or liquify but to decompose at temperatures between 185 for $\mathrm{Q}$ (Glutamine) to 280 for $\mathrm{H}$ (Histamine) For the other amino acids, they were not able to completely characterize the gases emitted. (Weiss et al, 2018)

There might be other more recalcitrant organics remaining but it seems that this should be sufficient to make sure no genetic material reaches the destination.

(Sauder et al, 2017 section 5.0) estimated that it would take 5-6 years to develop their automaton rover, at a cost of hundreds of millions of dollars, able to operate at $500{ }^{\circ} \mathrm{C}$. A rover able to operate at $300{ }^{\circ} \mathrm{C}$ would be easier to develop.

It's beyond the scope of this paper to explore whether higher temperatures are needed or whether this is a sufficient level of sterilization to prevent contamination of pre-biotic or early life. However if higher temperatures or more sterilization is needed, there are ways to do it.

\section{Designs for $500{ }^{\circ} \mathrm{C}$ to decompose organics if higher temperatures than $300{ }^{\circ} \mathrm{C}$ are needed}

Higher temperatures would also decompose the organics, so leaving nothing to contaminate the special region or subsurface ocean with biomolecules that could be incorporated in native life there. However they are more challenging to design for, and this may not be necessary depending on whether biomolecules that resist sterilization at $300{ }^{\circ} \mathrm{C}$ for months are of concern. 
High temperature sterilization at $500{ }^{\circ} \mathrm{C}$ for extended periods is feasible for small probes. It is more of a technical challenge for a complete surface rover with broadband communications, but that may also be possible in the future.

There have been several design studies for a rover that can withstand the higher temperatures of the Venus surface without cooling. This would need new components, and with this in mind, in 2007, NASA developed a silicon chip capable of 17,000 hours of continuous operation at $500^{\circ} \mathrm{C}$.

Landis has shown that solar cells can operate at Venus surface temperatures of $450{ }^{\circ} \mathrm{C}$ and tested them at those temperatures (Landis, 2013), and has sketched out a proposal for a camera to operate at up to $500{ }^{\circ} \mathrm{C}$ using similar principles to the imaging camera used for Viking (Landis, 2014).

Sauder et al have done a NASA concept study for an automaton rover using mechanical components similarly to the early mechanical ballistic computers that were used in the 1950s before the first digital computers, with a mix of mechanical components with occasional use of high temperature electronics but no centralized CPU. It would communicate either with a transceiver - but they say that this is not yet at a technological readiness level to send to Venus - or else using radar reflection from spinning disks with three orders of magnitude lower bit rates. They suggest that this idea could be used for $100 \%$ sterile rovers on Mars capable of visiting the RSL's perhaps deployed from a more capable but less fully sterlized master rover. (Sauder et al, 2017, section 6.2)

Brian Wilcox is working on a $100 \%$ sterile probe to descend into the Europan ocean. It would have vacuum insulation like a thermos flask, a blade that cuts ice chips that the body then melts and analysed. It would be heated to over $900^{\circ} \mathrm{F}\left(500^{\circ} \mathrm{C}\right)$ during its cruise to Europa which would not only kills microbes but also decomposes organics that would confuse the results.

In his abstract he says (Wilcox, 2017)

"A central thesis of this work is that we must start by addressing the Planetary Protection constraints, and not to try to add them on at the end. Specifically, all hardware in the probe would be designed to survive heat sterilization at $500{ }^{\circ} \mathrm{C}$ for extended periods, as required to meet the COSPAR 1-in-10,000 probability per mission of biological contamination of the ocean"

The NASA press release says (NASA, 2017)

"To ensure no Earth microbes hitched a ride, the probe would heat itself to over 900 degrees Fahrenheit (482 degrees Celsius) during its cruise on a spacecraft. That would kill any residual organisms and decompose complex organic molecules that could affect science results." 


\section{$\mathrm{CO}_{2}$ snow sterilization - alternative to temperatures high enough to decompose organics - MID EDIT}

An alternative to such high temperatures as $500{ }^{\circ} \mathrm{C}$ is to use $\mathrm{CO}_{2}$ snow. This is not enough to sterilize components on its own, but if the components are pre-cleaned then it can kill any remaining dormant microbes and not only that, also decompose the organics too. This could be done at the end of the journey after the heat sterilization phase.

Carbon dioxide snow is one of the few techniques that removes everything, if it is used to sterilize a part that is already reasonably sterile. This is a new technique for spacecraft sterilization currently being evaluated by ESA. See Deep cleaning with carbon dioxide. and Science Daily article about it. JPL are also exploring it. The main difficulty is scaling it up to sterilize a complete spacecraft. Also, although it is great at removing micron scale contamination, it's not so good at dealing with complete microbes.

So far, neither NASA nor ESA have approved it for planetary protection.

The great advantage of this is that it sterilizes at low temperatures, has no adverse effect on electronics, and also removes the organics completely if the robot starts off reasonably clean. It can also penetrate into tiny cracks and holes. It also has the additional advantage that it removes impurities that could interfere with the electronics.

If this method can be made $100 \%$ effective, you not only get no life on the spacecraft, but no DNA fragments or GTAs, or indeed, anything organic at all.

There are two ways to do it. One is to use supercritical liquid carbon dioxide, and enclose the instrument in a pressure vessel for small and delicate parts. The other way is to generate tiny particles of carbon dioxide snow which impact on the surfaces. See also wikipedia article on carbon dioxide cleaning.

With the supercritical liquid method, the liquid carbon dioxide penetrates into tiny holes, dissolves the organics, and then, as it escapes as snow, it takes the organics with it, and changes back to gas, so leaving the spacecraft completely dry with no residue.

The pressurized supercritical method is:

- Inject $\mathrm{CO}_{2}$ in a supercritical state. It behaves much like a liquid at 74 atmospheres upwards and $31 \mathrm{C}$.

- In this state, it dissolves organics readily

- It then forms snow which captures the organics and gets blown / sucked away.

- The snow evaporates rapidly into the gas state, leaving no residue

- Can be mixed with Hydrogen peroxide and other chemicals to increase effectiveness.

- Can be used even with sensitive electronics.

- It has been used to clean USB drives in testing and they functioned afterwards.

- Surface is left with no trace of organics, not even dead organisms. Major plus! 
The impacting snow method is what ESA are investigating and use already in an operating spacecraft clean room. It relies on tiny explosions of carbon dioxide snow to clean the spacecraft.

They mix the liquid $\mathrm{CO}_{2}$ in advance with clean dry air, where it forms tiny snowflakes. So they then hit the spacecraft with minute snowflakes which penetrate into every nook and cranny. The spacecraft surfaces are relatively hot for the snowflakes. So when they hit those "hot" surfaces, they suddenly expand 800 fold, in mini explosions, taking the organics and other matter away with them. Instead of injecting with supercritical liquid which forms snowflakes later, they hit the surface with $\mathrm{CO}_{2}$ that has already formed into snowflakes, soon after it left the nozzle.

They plan to use this method to help with sterilizing ExoMars due for launch in 2018. See this press release from August 2015.

"The method originates from the USA, and is used to remove paint from aircraft fuselage. A powerful jet of frozen carbon dioxide $\left(\mathrm{CO}_{2}\right)$ crystals, about the size of a rice kernel, blasts the paint right off the metal. The researchers made this crude instrument substantially more refined. Instead of $\mathrm{CO}_{2}$ pellets, they use carbon dioxide snow to work on each individual component - from the highly sophisticated aluminum workbench to the ring washers. Here's the rub: the beam that the jet emits is additionally accelerated with a blast of CDA (clean dry air) that encases it. This is how it penetrates into every nook and cranny, removing even the minuscule pollutant. As soon as the tiny snowflakes hit the relatively hot surface, they become gaseous, causing their volume to explosively expand 800-fold. The detonation pressure completely sweeps away every single bit of dust, even fingerprints which the cold gas had just turned brittle. "This approach involves a dry process that does not warp surfaces. When cleaning, these can be gently treated with $\mathrm{CO}_{2}$. That makes it unnecessary to apply heat or chemicals," Gommel says when explaining the advantages of this method."

More about planetary protection for ExoMars here.

This shows scientists using a similar technique to clean all the organics from the surface of a test mirror:

Scientists using $\mathrm{CO}_{2}$ snow cleaning to clean a test mirror - if the James Webb mirror gets contaminated by organics they can clean it in this way before the launch. The advantage of this approach is that it doesn't just sterilize the equipment. It also removes all trace of organics.

We could use this, first on Earth before the final heat sterilization stage.

We could also take a container of $\mathrm{CO}_{2}$ and use it for a final $\mathrm{CO}_{2}$ snow sterilization stage, or in the case of Mars, use carbon dioxide in the Mars atmosphere for an extra $\mathrm{CO}_{2}$ snow sterilization stage after landing there. 
Of course this adds somewhat to the complexity of the mission. But if you value the Europa ocean highly, then it's worth it, to make sure we have no practical chance of making life there extinct.

One advantage of $\mathrm{CO} 2$ snow is that it is especially easy to sterilize microsats, and microrovers, to the extent that there are no organics left.

Another approach, useful for microrovers that will re-enter an atmosphere, such as the Mars atmosphere, is to pre-sterilize the entire reentry spaceship and enclose it in plastic before the launch. The plastic cover would then simply burn away during re-entry exposing the sterile spacecraft inside.

Staehle et al. propose this method for their miniature paraglider to run piggyback on larger missions. It would re-entry as a parawing glider, capable of 10+ km of guided flight at a 3:1 glide ratio, with the entire spacecraft massing 10-20 kg including deployer and de-orbit propulsion with science payload 1-2 $\mathrm{kg}$ and cost $\$ 20 \mathrm{M}$ reducing to $\$ 10 \mathrm{M}$ if 2-3 copies are made (Staehle et al 2015)

\section{Sterilizing in situ life detection experiments that need amino acids, polyclonal antibodies and other delicate components}

What about organics and other sensitive components of the experiments themselves? The chiral labeled release experiments needs to have chiral amino acids to feed to the microbes to test for presence of a metabolism. SOLID3 needs its polyclonal antibodies.

Most organics can't survive high temperatures. Glutamine, one of the less heat stable amino acids, decomposes thermally at $185 \mathrm{C}$ (Weiss et al, 2018).

Viking did use amino acids, but a lower temperature for heat sterilization. So maybe we just have to choose a temperature low enough so that the amino acids and other organics survive the heat. It would still be sterile of all life, but with less decomposition of the organics, so then we would be more reliant on other methods to remove those. The amino acids would also lose their chirality for the chiral labeled release at lower temperatures than the temperatures that decompose the amino acids.

So, the need for $100 \%$ sterilization might restrict some of the possibilities for in situ biological experiments. Or could those organics be transferred in some way to the spacecraft after it is sterilized, or manufactured in situ?

We could also use a sterile rover or lander to collect a sample and return it to less sterile instruments at some distance from the site of interest. This might work for small special regions such as RSL's on Mars, or it could be returned to orbit for immediate analysis and then returned to Earth. But this reduces some of the advantages of in situ study, the ability to 
examine many samples and study them in situ in a short period of time, many more than could be returned for study, also to use the results in decisions about what to sample next.

With Europa's low gravity and fairly low orbital velocity, and especially with Enceladus, with hardly any gravity and very low orbital velocity, it might be relatively easy to return samples to orbit for the less well sterilized instruments to study.

Perhaps another approach might be to have most of the spacecraft sterilized using heat sterilization but a separate section, perhaps a tank with these organics, insulated from the rest of the spacecraft and pre-sterilized using another method that is effective enough for a simpler component such as a tank. $\mathrm{CO}_{2}$ snow sterilization might be a good way to achieve that too.

If the rover will re-enter an atmosphere, for instance for Mars, the easiest way to handle temperature sensitive organics might be the idea from the last section to pre-sterilize the entire rover using $\mathrm{CO} 2$ snow (after first adding the organics in sterile form inside of a container), including re-entry vehicle, and enclose it in a plastic cover that burns away during re-entry. (Staehle et al 2015)

\section{Biosphere collisions can be mutually beneficial as well as harmful}

So far we've focused on situations where the biosphere collisions are harmful, either mutually or in one direction. However we should also recognize that the collisions can also be beneficial.

Here on Earth, invasive species aren't always harmful. Schlaepfer et al did a survey of invasive species and in their table 1 they find many non native species that are actually beneficial. Some were deliberately introduced for their value for conservation. Many of the best examples were introduced unintentionally. (Schlaepfer et al, 2011). Here are some of their categories.

- Habitat, shelter, and food for native species (e.g. non native tamarisk as nesting sites for nesting birds, and non native plants in California for native butterflies to lay their eggs and for the caterpillars to feed).

- Catalysts for restoration - e.g. non native guava trees in Kenya support fruit eating birds and encourage seed dispersal leading to forest restoration

- Ecosystem engineers - e.g. non-native sea squirts (ascidian) in intertidal waters in Chile creating three dimensional matrix that increases species richness

- Taxon substitution - e.g. Aldabra giant tortoise replacing extinct Cylindraspis giant tortoise in the Mascarene island (this one was intentional) 
This could happen in the forwards direction to Mars or indeed backwards direction to Earth too.

For example, introduced lichens, if Mars has never evolved them, could provide habitat, shelter (from the UV) and food, as a prime producer, using just carbon dioxide, water vapour, and trace elements from the basalt. If Mars had lichens in the past and they went extinct, it could help through taxon substitution.

Cockell gave an example of another beneficial interaction, that if a martian species was at risk of going extinct through lack of nitrogen we might be able to prevent its extinction through introducing nitrogen fixating microbes from Earth (Cockell, 2005).

If this nitrogen fixation was combined with photosynthesis, it could convert many previously uninhabited habitats on Mars into habitats that are also habitable to the native life there by providing them with organics, nitrates, and shelter in biofilms. Cyanobacteria could do this, or haloarchaea, or Lichens or any other photosynthetic prime producer. If nitrogen fixing is important the lichens need to be cyanolichens as the cyanobacteria photobiont component of the lichen then will do the nitrogen fixing_(Seneviratne et al, 2006).

This could expand the range of habitats for native life, and encourage its diversity.

Cockell suggests an approach of "biorespect" rather than preservation (Cockell, 2005):

"The ethical approach suggests that contamination is acceptable. The shedding of microorganisms from human habitats and space suits, microorganisms which themselves originate from unsterilizable humans, is acceptable provided that they do not destroy the integrity of indigenous microbial communities."

He argues that many species of microbes from Earth would be able to survive only in certain restricted habitats on Mars because they required a limited range of conditions such as $\mathrm{pH}$, chemical tolerances, and temperature. However he urges caution for Europa because of the globally connected ocean. This is from 2005 before the Phoenix results, discovery of the RSLs and so on at a time when it seemed that any habitats on Mars must be below the surface at geological hot spots and probably localized to small oases.

The modern picture of Mars is more globally connected, through the dust storms, because of the surface thin brines that may well be habitable in many locations on Mars. They may be similar enough for the same microbe to inhabit them all, and we also know of many polyextremophiles like chroococcidiopsis that are versatile and could survive in many different microhabitats on Mars.

It might be that humans, by going to Mars and shedding micro-organisms, just provide a wider range of habitats for martian life - and that in turn the martian life never harms us either but is only beneficial in its effects on Earth life too. Perhaps it may be a wonderful symbiosis of the two biospheres. 
However, as we have seen in this paper, there are enough biosphere interactions that could go very wrong, that we need to be careful. Much may depend on the outcome of this biosphere collision.

\section{Earth using us to reach into space and "it will be magnificent" - Robert Zubrin}

Robert Zubrin has an argument which he presented in the "Making of" episode 0 of season 1 of the National Geographic series Mars (Zubrin, 2016).

"I would say that we have not only the right, but the obligation, to go and establish ourselves on Mars. We are the creatures with all of our flaws that the Earth's biosphere has evolved to allow itself to reach out and establish itself on additional worlds. And we will take this nearly dead world and we will create a fully living world there. And so there'll be new species of birds and fish and plants. And it will be magnificent. No-one will be able to look on it and not feel prouder to be human."

We provide a way of getting into space, yes. But if Earth evolved us to reach out and establish itself on other world,s, it also evolved us with the intelligence, foresight, and deep scientific and ethical understanding to guide that exploration. We are the Earth's biosphere's guiding intelligences in space, and that may be one of our main roles.

For instance one of the main reasons for going into space may be to protect Earth from hazards (such as asteroids), or to find resources for use on Earth, or to increase our understanding of ourselves, and of science, biology and the universe, or indeed, as a place for adventure and recreation. We have already found many benefits for Earth's biosphere, already, through our satellites in Earth orbit.

It's not automatic that what anything humans find inspiring and want to do is going to work, and is going to be harmless to ourselves or to others. For instance deliberate introduction of the European wild rabbit into Australia for sport led to many problems_(Fenner, 2010).

Also, the solar system is vast with many other places of great interest where we can visit, and even set up home, without risk of contaminating them.

Yes, we can set up those magnificent habitats that Robert Zubrin talks about in settlements constructed from materials in the asteroid belt. There is enough by way of materials there to create habitats with total living area equal to that of a thousand times the land area of Earth. We can also set them up on Callisto, and in the lunar caves, which may be over 100 kilometers long and up to 5 kilometers in diameter in the low lunar gravity.

We could paraterraform Titan if there is no native life there to be made extinct, more easily than anywhere. It has abundant wind and hydro power which could power electric lighting for agriculture in vast habitats that straddle the entire moon. 
So, yes, we could set up habitats in space and indeed they could be magnificent. But there is no urgent need to go to Mars right away to do this. There are many other places to try it, and given the special planetary protection issues for Mars, we can take the time to study Mars first and decide what to do based on what we find there.

\section{What if the decision is to keep Mars, Europa or Enceladus biologically isolated for ever?}

What if we find independently originated early life on Mars, Europa or Enceladus, or intricate prebiotic chemistry, or some other form of life that Earth life would make extinct? Should we leave these destinations pristine to avoid contaminating it?

In the other direction also, if we find something in any of these places that endangers the environment of Earth, should we keep them biologically isolated from Earth indefinitely?

Either of these could lead to a future where serious consideration is given to the need for biological isolation of Earth from Mars.

The backwards direction is easier to argue. In that case it might depend on whether it is a major or minor nuisance. But even a relatively minor nuisance such as fridges that have to operate at $-80 \mathrm{C}$ would probably have most humans arguing against relaxing restrictions.

In the forwards direction, then it would be like having our own exoplanet, with its distinct biology, in our own solar system. The nearest terrestrial planet like that, other than Mars, may be light years away. Depending on future technological progress, it might be centuries before we have a similar opportunity - or if life is rare in our galaxy, maybe even millions of years. Some might argue however, that once we fully understand the life on Mars we can find a way to replicate it in space habitats or in biologically isolated habitats for martian life on the planet.

We can expect much debate in either of those two cases. However, on the basis of what is known so far, it is a possible outcome of our studies that the decision is made to keep Mars, Europa or Enceladus biologically isolated from Earth.

This would be a future where you have agile rovers on the surface. and increasingly sophisticated humanoid avatars on the surface as well, directed and teleoperated by colonists in orbital colonies. It is a future where the Martian past and present turn out to be so interesting (or so hazardous to Earth's environment), that humans never land on the surface in person, in their physical bodies, to preserve a biologically pristine Mars.

\section{We are our biosphere's noosphere - its thinking component}


We are our biosphere's way of getting into space and visiting other planets as Zubrin suggests. But we are also its noosphere (de Chardin 1932), its intelligence that it can use to avoid potentially catastrophic consequences. We already have used this, for instance to prevent extinction of blue whales, and to feed billions through the green revolution. By acting as a thinking biosphere, we can plan our missions in space in such a way as to keep our options open, and to advance into space as humans and robots together, each doing what we do best, with the robots as our eyes, and hands, in regions we cannot yet explore in person.

If it is true that other biospheres are as potentially vulnerable as this article suggests, perhaps this is a time to start a serious development program with the aim of $100 \%$ sterile landers and rovers for astrobiological exploration. This would be a joint program with the Venus rover project.

Although this would take some time and would involve some expense, the pay off would be huge. With $100 \%$ sterile rovers we could then send our robotic astrobiological explorers anywhere in our solar system, including the depths of Enceladus and Europa, without any more concerns about forward contamination.

Once we have $100 \%$ sterile rovers, if we have Elon Musk's BFR to send 150 tons to Mars orbit in one go, then the astrobiological exploration phase could develop quickly. The BFR couldn't land on Mars itself as it can't be sterilized. However, its 150 tons per payload would be enough to have several tons of equipment landed on each of over a dozen landing spots on Mars. Also, with two BFRs in orbit around Mars connected by tethers and spinning for artificial gravity we'd have an instant space station to permit dozens of people to explore the Mars surface via telepresence. By sending many instruments to study Mars in situ from Earth first, and then from orbit, we could have the results from astrobiological instruments that have directly studied all the known surface habitats on Mars in diverse locations with just one human mission to Mars orbit. It would be bound to turn up more questions to ask, more instruments to send. But within a decade we'd have advanced our understanding of Mars astrobiology hugely.

At that point we'd be able to make decisions wisely. There might well be many views on what we do next but at least we'd make the decisions based on knowledge rather than guesswork.

Meanwhile at our current level of technology, we already have a way to study Enceladus and Europa with flyby missions that collect samples from the plumes, to study in situ immediately after collection, with no risk of contamination in either direction, and search for unambiguous traces of life there.

On Mars we can continue to search for past life, and search for spores of past life in

This would seem to impact on the hopes of Mars colonists. But is it not important to evaluate the effects of Earth's biosphere on Mars and vice versa before proceeding? 
This is not a rubber stamping exercise. Yes it's possible that Earth and Mars life gets on nicely together in a wonderfully symbiotic joining of biospheres.

But it is also possible that there's a clash in which one or other or both biospheres suffer hugely, to the point of degrading the environment of Earth, possibly to the point of mass extinctions, and in the forward direction possibly to the point of complete extinction of native martian life. We have as yet no sure way to assess which of these we face in the case of a collision with the biospheres of Earth and Mars. We need to know this before we can make our decisions wisely.

\section{Value of experimental biosphere collisions in large kilometer scale habitats first}

One way to help eliminate many of the unknowns involved in introducing life to a new planet or moon would be to start by experimenting first with larger and larger habitats. They are far more controllable, as unlike a planet, they have a "reset button". If necessary you sterilize the whole thing and start again.

With habitats made from materials from asteroids and comets, spinning for artificial gravity, we can experiment with any level of gravity, and use thin film mirrors to reflect sunlight into it at varying intensities, and vary the day length. It can have miniature lakes as well as "oceans" of salty water too. This is a level of technology much more accessible than terraforming. We could take such a habitat to completion in a decade or less.

Once such habitats are available, varying the gravity level, insolation, day night cycle, ocean, atmospheric and soil chemistry would be an easy matter. If desired we can simulate perchlorate soils, even the Martian atmosphere and dry ice geysers, not in a small simulation chamber but a large habitat. Any desired atmospheric, ocean, and soil conditions can be simulated. It would be a natural step by step future development from the current small Mars simulation experiments left on the exterior of the ISS for BIOMEX, part of Expose R2 (Gronstal, 2014).

We can also set up large habitats to parateraform moons and planets with no risks of forward contamination. These can include Callisto, Titan, possibly the Venus upper atmosphere, and nearest to us, the Moon with its lava tube caves, that may be up to 5 kilometers in diameter and a hundred kilometers long, larger than an O'Neil habitat. All of these have potential for paraterraforming, using technology that is far more accessible to us at present than the megatechnology planet scale mirrors and other methods suggested for Martian terraforming.

For example, if a destination turns out to be lifeless, with uninhabited habitats, perhaps we should not be in a hurry to seed a lifeless Europa, or indeed, Mars? We want to understand what happens in such a huge habitat without life. How far does it get towards life? Is there perhaps some primitive almost alive organism that's not quite life, or not reproducing exactly, in its hydrothermal vents or some surface habitat? Are there multiple sporadic attempts at biogenesis that constantly then go extinct again? Do we want to seed it with modern life, or 
do we attempt at triggering a new abiogenesis by repeatedly seeding it with plausible protocells, or leave it "as is"?

We covered Cassie Conley's example of certain microbes that, if introduced to Mars, would turn the underground aquifers to cement in the methane rich conditions there. If there is no life this won't have happened. Is this what we want to do to the planet? Is there some form of life we could introduce first to prevent it?

As another example, a natural way to try to warm Mars up would be to engineer it with microbes that produce hydrogen sulfide, poisonous to humans. Do you engineer such microbes and turn Mars into a planet that smells like a sewer but is a little warmer for indigenous life or introduced life? Maybe warm enough for liquid water especially if the $\mathrm{H}_{2} \mathrm{~S}$ thickens the atmosphere too through $\mathrm{CO}_{2}$ release + the $\mathrm{H}_{2} \mathrm{~S}$ itself?

Or, maybe you don't want that to happen, but if so how do you direct it into some other direction? What about long term cycles like carbon dioxide recycling?

Then, human pathogens can also escape into the biosphere, evolve, and may return to us with new capabilities. The atmosphere or the water may itself become toxic to us as in the example of hydrogen sulfide generating microbes, or cyanobacteria producing BMAA and toxins that can kill cows. It may produce allergens, protoxins and so on.

This may be something that is under our control, if we introduce life to the biosphere in the correct order. But we may need to undertake experimental research first to find the optimal way to do it.

Introducing life to habitats is experimental science because the environment is controllable and we have a "reset button". We can run controls, we can try varying the conditions and so on. Introducing life to a planet is not so much an experiment, as an irreversible bioengineering project with commitments that stretch for millennia into the future or longer.

It may be that it takes careful planning, to ensure that colliding biospheres can do this in a symbiotic way that is mutually beneficial. If we find a second biosphere on Mars, we can experiment with biosphere collisions on a smaller scale first in large space habitats, in locations where there is no risk of either Earth or the other biosphere (Mars, Europa, Enceladus etc) being directly affected.

\section{Seeding Earth life on exoplanets}

The physicist Claudius Gros looks at a clash of interpenetrating biospheres in his paper on a "Genesis project" to develop ecospheres on transiently habitable planets, (Gros, 2016). As we mentioned before he suggested the worst case scenario is that both the host planet and any introduced multicellular life from Earth is reduced to a microbial slush due to the inability of either type of multicellular life to recognize the microbes from the colliding biosphere as "non self". 
"In the worst case scenario more or less all multicellular organism of the planet targeted for human settlement would be eradicated. The host planet would then be reduced to a microbial slush in a pre-cambrian state, with considerably prolonged recovery times. The leftovers of the terrestrial and the indigenous biospheres may coexist in the end in terms of 'shadow biospheres' "

In his paper he suggests sending microbes to planets that will be habitable only briefly. From our experience on Earth, it took billions of years for the first eukaryotes (cells with a nucleus) and multicellular organisms to arise. So what if we were to try to kickstart all that?

To do this, first, he suggests, we find a planet that has only a few hundred million or a billion years of habitability left - still plenty of time for higher lifeforms, with future prospects not unlike our Earth - but it's got no life there yet. Then the idea is to seed it with a few eukaryotes and other higher lifeforms from Earth. Hopefully that then saves its ecosystem billions of years of evolution and means that new complex life will arise there that would never have done so without our intervention.

He would look for a planet with no life at all, to avoid issues of clashing biospheres and incompatible biochemistry. It would be a very long term experiment, indeed, unusually, it wouldn't matter how long it takes to get there, so long as the process can be automated. The aim is to make the planet into one with complex life, and perhaps intelligence, hundreds of millions of years into the future, so a few extra centuries now will make no difference.

This raises many ethical questions, as well as practical ones

- How do we know that there is no life there yet? What if it is cryptic, hidden from view unless you examine the planet close up, or has only just started to evolve?

- Is it true that life always takes billions of years to evolve multicellular life as it did on Earth?

- It's bound to have life precursor complex molecules, as even meteorites and comets have those. Perhaps it will have protobionts too, almost alive life precursors. Might we be interfering in the early stages of development of a form of biology that we don't understand yet?

- If we introduce new life to a planet, do we not have some responsibility for the creatures that will arise there billions of years from now? Maybe what we think will be a stable biosphere turns out to be a disaster for them, just as they evolve to complex intelligent life like ourselves?

- What if the planet then evolves intelligent creatures that turn out to be a harmful to our galaxy?

- Is it always a good thing to increase the amount of complex life, and intelligence, in our galaxy and universe?

On the other hand, if we knew what we were doing, perhaps it is a beneficial thing to do, bringing life to an entire biosphere which wouldn't be there otherwise. In that case, it's not unlike bringing a desert to life. The idea of bringing new life to a desert certainly has its appeal and it seems a worthwhile thing to do, somehow, maybe even irrespective of whether 
any humans or other intelligent beings enjoy it? However the responsibilities to those future biospheres are high and perhaps to do this successfully is something that needs more experience than we have quite yet.

\section{Aim for "win win" rather than uneasy compromise between colonization enthusiasts and astrobiologists}

Meanwhile there is much humans can do on the Moon. The spacesuits and habitats designed for Mars also work on the Moon. Whatever we do further afield, the Moon is bound to be the main place tourists visit outside of Earth for the foreseeable future, and a science hub, and it is of astrobiological interest too. If we get human settlers anywhere in our solar system, there are bound to be some on the Moon. So why not start our settlement experiments there? Sending humans into space is hard. The Apollo astronauts were test pilots that made decisions in seconds with a cool head that would have killed less able astronauts. Returning to the Moon is dangerous enough at present, and we don't need to make it more exciting and dangerous than it is already.

The Moon is of astrobiological interest too. It collects meteorites as readily as Earth, and should have meteorites from early Earth, Mars, Ceres and possibly Venus too. The amounts may be substantial. According to one estimate, 7 ppm from Earth. A 10 by 10 square kilometer area of the Moon may have 20 metric tons of materials from early Earth. The materials transferred from Venus over the same area may be 1-30 kg and from Mars as much as $180 \mathrm{~kg}$ (Armstrong et al, 2002).

If any of those places had life, the organics may be preserved on the Moon at liquid nitrogen temperatures in the permanently shaded craters at its poles. These also present no risk of forward or backwards contamination. The lunar caves are also of great interest and the Moon may have many surprises in store for us.

Once we have the ability for safe interplanetary travel then humans could study Mars from orbit via telepresence with both astronauts and the public engaged in the in situ search for life. Further afield, the natural place for humans in Jupiter's system is not Europa, but Callisto, outside of the main radiation belts, with lower levels of ionizing radiation even than Mars, with abundant ice as well as deposits from carbonaceous meteorites, and other resources. Although it has a deep subsurface ocean, the surface is ancient, unchanged for billions of years, and it is classified as planetary protection category II similar to the Moon(Adams et al, 2003).

In the Saturn system the natural place for humans is Titan, which has vast potential for both wind and hydropower. The atmosphere protects not just from ionizing radiation and micrometeorites but even large meteorites, similarly to Earth's atmosphere. A human there would not need a spacesuit, often likened to a miniature spaceship in its own right. They just need a closed system rebreather and insulation from the cold. Even in those cold conditions, adequate insulation is well within our capabilities, and is similar in technological sophistication to the equipment you buy in an outdoor sports shop, and this moon has 
abundant raw materials suitable for making plastics. This would depend on planetary protection assessment, for instance, does it have cryovolcanoes? But if it is suitable then it could be one of the easiest places for humans to live outside of the Earth.

There are the moons of Mars also, Mercury, possibly airships in the clouds of Venus, and many asteroids (though Ceres may have planetary protection considerations as a result of the observation of water vapour plumes). When it comes to potential settlement, the asteroid belt has materials sufficient to make habitats (spinning for artificial gravity) with a total habitable surface area of a thousand times the land area of Earth. These "thousand planets" can be constructed on timescales of a decade or less per settlement. Human settlements further away from the sun are also possible with thin film mirrors to concentrate the sunlight out to Pluto and beyond. Such a future civilization would be immune to anything that could happen in our solar system for billions of years right through to the red giant phase and final end state of the sun as a white dwarf, by moving the settlements further away or closer as needed.

In this way - let's not move into a future of uneasy compromises where Mars colonization enthusiasts struggle but fail to keep Earth contamination contained to their settlements and astrobiologists out of frustration plan rapid astrobiological surveys that they know will cause extensive forward contamination.

Meltzer put it like this (Meltzer, 2012 page 479)

"We humans have a burning desire to increase our understanding of everything around us, but we are accountable to future generations of scientists to explore our solar system without destroying the capability of others to conduct their own investigations. The planetary protection field is thus driven by a deep regard for the importance of both present and future scientific inquiry. Careless planetary exploration in the present could forever obfuscate the answer to a vital question: Are we Earthlings alone in the universe?"

Let's move to a "win win" situation. Let's be even more ambitious with humans, aim to send them as far as Jupiter, Saturn and beyond, study Mars from orbit, explore the Moon - and at the same time, let's be equally ambitious with our astrobiological searches, to aim for $100 \%$ sterile landers and rovers, where necessary.

In that way we can leave our children with a solar system with the same opportunities and the same heritage that we received ourselves. And if we do send humans to these places, let's do it in knowledge and understanding of what is there and what our microbes will do to it, and indeed, what its native life will do to us. And if we are going to lose something as a result of sending humans there, let's at least know what it is we are going to lose, before we lose it. Let's make our decisions based on knowledge of what the true situation is there.

\section{Bibliography}


Abad-Manterola, P., Burdick, J., Nesnas, I.A. and Cecava, J., 2009, March. Wheel design and tension analysis for the tethered axel rover on extreme terrain. In Aerospace conference, 2009 IEEE (pp. 1-8). IEEE.

Abdel-Nour, M., Duncan, C., Low, D.E. and Guyard, C., 2013. Biofilms: the stronghold of Legionella pneumophila. International journal of molecular sciences, 14(11), pp.21660-21675.

Abe, S., 2001, Can Liquid Water Exist on Present-Day Mars? NASA Astrobiology Institute Abrevaya, X.C., Mauas, P.J. and Cortón, E., 2010. Microbial fuel cells applied to the metabolically based detection of extraterrestrial life. Astrobiology, 10(10), pp.965-971.

Adams, F.C. and Spergel, D.N., 2005. Lithopanspermia in star-forming clusters. Astrobiology, 5(4), pp.497-514.

Adams, R.B., Alexander, R.A., Chapman, J.M., Fincher, S.S., Hopkins, R.C., Philips, A.D., Polsgrove, T.T., Litchford, R.J., Patton, B.W. and Statham, G., 2003. $\underline{\text { Conceptual design of }}$ in-space vehicles for human exploration of the outer planets.

Aerts, J.W., Röling, W.F., Elsaesser, A. and Ehrenfreund, P., 2014. Biota and biomolecules in extreme environments on Earth: implications for life detection on Mars. Life, 4(4), pp.535-565.

Agriculture Victoria, Note Number: AG1093, Blue-green algal poisoning of stock

Aharonson, O. Evolution of design for mini cryobot to explore the Mars polar ice deposits.

Alberts B, Johnson A, Lewis J, et al. Molecular Biology of the Cell. 4th edition,2002, New York: Garland Science; .Cell Biology of Infection

Ammann, Walter and Barros, John and Bennett, Allan and Bridges, Jim and Fragola, Joseph and Kerrest, Armel and Marshall-Bowman, Karina and Raoul, Hervé and Rettberg, Petra and Rummel, John and Salminen, Mika and Stackebrandt, Erko and Walter, Nicolas (2012) Mars Sample Return backward contamination - Strategic advice and requirements Report from the ESF-ESSC Study Group on MSR Planetary Protection Requirements.

European Science Foundation, archived copy here. (Printing: Ireg - Strasbourg). ISBN 978-2-918428-67-1.

American Chemical Society, 2015, “Cyborg bacteria outperform plants when turning sunlight into useful compounds", Phys.org

"A future direction, if this phenomenon exists in nature, would be to bioprospect for these organisms and put them to use," 
Anbar, A.D. and Levin, G.V., 2012, June. A Chiral Labeled Release Instrument for In Situ Detection of Extant Life. In Concepts and Approaches for Mars Exploration (Vol. 1679, p.4319)

Armstrong, J.C., Wells, L.E. and Gonzalez, G., 2002. Rummaging through Earth's attic for remains of ancient life. Icarus, 160(1), pp.183-196.

Bada, J.L., Aubrey, A.D., Grunthaner, F.J., Hecht, M., Quinn, R., Mathies, R., Zent, A. and Chalmers, J.H., 2009. Seeking signs of life on Mars: In situ investigations as prerequisites to a sample return mission. Planetary science decadal survey White Paper, Scripps Institution of Oceanography, USA.

Barry, J.R., Jenkins, D.R., White, D.J., Goodman, P.A. and Reingold, L.A., 2003. Columbia Accident Investigation Board Report. Volume Two. See Appendix D10, Debris recovery

Baugh, R.F., 2017. Murky Water: Cyanobacteria, BMAA and ALS. Journal of Neurological Research and Therapy, 2(1), p.34.

Belbruno, E., Moro-Martín, A., Malhotra, R. and Savransky, D., 2012. Chaotic exchange of solid material between planetary systems: implications for lithopanspermia. Astrobiology, 12(8), pp.754-774.

Steven a. Benner; Paul Davies (15 Nov 2012). Chris Impey; Jonathan Lunine; José Funes, eds. Towards a Theory of Life, in Frontiers of Astrobiology (Page 37). Cambridge University Press. ISBN $\underline{9781139789226 .}$

Biello, D., 2016. Bionic leaf makes fuel from sunlight, water and air. Sci. Am.

Blackmond, D.G., 2010. The origin of biological homochirality. Cold Spring Harbor perspectives in biology, 2(5), p.a002147.

Board, S.S., 2000. Preventing the forward contamination of Europa. National Academies Press.

Board, S.S. and National Research Council, 2002. The Quarantine and Certification of Martian Samples. National Academies Press.

"The initial processing of returned martian samples should be restricted to a $B S L-4$ laboratory in the quarantine facility. A very modest gas-tight glove box (Class III cabinet) in a "clean room" (class 10; however see following g section) will be sufficient for this purpose. " page $\underline{51}$

Board, S.S. and National Research Council, 2009. Assessment of planetary protection requirements for Mars sample return missions. National Academies Press. page 48 
"Despite suggestions to the contrary, it is simply not possible, on the basis of current knowledge, to determine whether viable martian life forms have already been delivered to Earth. Certainly in the modern era, there is no evidence for large-scale or other negative effects that are attributable to the frequent deliveries to Earth of essentially unaltered martian rocks. However the possibility that such effects occurred in the distant past cannot be discounted."

Board, S.S. and National Research Council, 2007. An Astrobiology Strategy for the Exploration of Mars. National Academies Press.

Boston, P.J., 2010. Location, location, location! Lava caves on Mars for habitat, resources, and the search for life. Journal of Cosmology, 12, pp.3957-3979.

Bostrom, N., 2002. Existential risks: Analyzing human extinction scenarios and related hazards.

Boxe, C.S., Hand, K.P., Nealson, K.H., Yung, Y.L. and Saiz-Lopez, A., 2012. An active nitrogen cycle on Mars sufficient to support a subsurface biosphere. International Journal of Astrobiology, 11(2), pp.109-115.

Boyle, R.A., Lenton, T.M. and Williams, H.T., 2007. Neoproterozoic 'snowball Earth'glaciations and the evolution of altruism. Geobiology, 5(4), pp.337-349.

Brazil, R., 2015, The Origin of Homochirality, Chemistry World.

Brown, G.D., Denning, D.W., Gow, N.A., Levitz, S.M., Netea, M.G. and White, T.C., 2012. Hidden killers: human fungal infections. Science translational medicine, 4(165), pp.165rv13-165rv13.

Brown, J.R., 2003. Ancient horizontal gene transfer. Nature Reviews Genetics, 4(2), p.121.

Bryson, P.D., 1996. Comprehensive reviews in toxicology: for emergency clinicians. CRC press. See page 680

Cartwright, J.H., Piro, O. and Tuval, I., 2007. Ostwald ripening, chiral crystallization, and the common-ancestor effect. Physical review letters, 98(16), p.165501.

Chyba, C., 2002, September. Planetary Protection: Two Relevant Terrestrial Examples. In Bulletin of the American Astronomical Society (Vol. 34, p. 839).

Chin, J.P., Megaw, J., Magill, C.L., Nowotarski, K., Williams, J.P., Bhaganna, P., Linton, M., Patterson, M.F., Underwood, G.J., Mswaka, A.Y. and Hallsworth, J.E., 2010. Solutes determine the temperature windows for microbial survival and growth. Proceedings of the National Academy of Sciences, 107(17), pp.7835-7840. 
Cichan, T., Bailey, S.A., Antonelli, T., Jolly, S.D., Chambers, R.P., Clark, B. and Ramm, S.J., 2017. Mars Base Camp: An Architecture for Sending Humans to Mars. New Space, 5(4), pp.203-218.

Cockell, C.S., 2005. Planetary protection-A microbial ethics approach. Space Policy, 21(4), pp.287-292.

Cleland, C.E., 2007. Epistemological issues in the study of microbial life: alternative terran biospheres?. Studies in History and Philosophy of Science Part C: Studies in History and Philosophy of Biological and Biomedical Sciences, 38(4), pp.847-861.

Cockell, C.S., 2008. The Interplanetary Exchange of Photosynthesis. Origins of Life and Evolution of Biospheres, 38(1), pp.87-104.

Cockell C.S., Balme M., Bridges J.C., Davilad A., and Schwenzer S.P., 2012, Uninhabited habitats on Mars. Icarus 217:184-193. Crossref, Google Scholar

Cockell, C.S., 2014. Trajectories of martian habitability. Astrobiology, 14(2), pp.182-203.

Cooper, G. and Rios, A.C., 2016. Enantiomer excesses of rare and common sugar derivatives in carbonaceous meteorites. Proceedings of the National Academy of Sciences, 113(24), pp.E3322-E3331.

Cornish-Bowden, A. and Cárdenas, M.L., 2017. Life before LUCA. Journal of theoretical biology, 434, pp.68-74.

"The earliest self-sustaining organisms, which existed before LUCA, must have been vastly simpler than anything we can see today, and the first catalysts cannot have been as specific as protein enzymes"

COSPAR, 2011. COSPAR Planetary Protection Policy, 20 October 2002, As Amended to 24 March 2011.

Cousins, C.R. and Crawford, I.A., 2011. Volcano-ice interaction as a microbial habitat on Earth and Mars. Astrobiology, 11(7), pp.695-710.

Czaja, A.D., Beukes, N.J. and Osterhout, J.T., 2016. Sulfur-oxidizing bacteria prior to the Great Oxidation Event from the 2.52 Ga Gamohaan Formation of South Africa. Geology, 44(12), pp.983-986. See also Czaja interviewed for University of Cincinatti by Melanie Schefft, 2016, Life before oxygen,

"And this discovery is helping us reveal a diversity of life and ecosystems that existed just prior to the Great Oxidation Event, a time of major atmospheric evolution."

Danovaro, R., Dell'Anno, A., Pusceddu, A., Gambi, C., Heiner, I. and Kristensen, R.M., 2010. The first metazoa living in permanently anoxic conditions

. BMC biology, 8(1), p.30. 
David, L., 2017, Mars Flows: A Recurring Controversy, Leonard David's "Inside Outer Space" blog (space journalist).

Davies, P., 2014, The key to life on Mars may well be found in Chile, The Guardian

Davila, A.F., Skidmore, M., Fairén, A.G., Cockell, C. and Schulze-Makuch, D., 2010. New priorities in the robotic exploration of Mars: the case for in situ search for extant life.

Astrobiology, 10(7), pp.705-710.

de La Torre, R., Sancho, L.G., Horneck, G., de los Ríos, A., Wierzchos, J., Olsson-Francis, K., Cockell, C.S., Rettberg, P., Berger, T., de Vera, J.P.P. and Ott, S., 2010. Survival of

de Chardin, P.T., 1923, "Hominization", 1957. Included in. "The Vision of the Past", p.63. "And this amounts to imagining, in one way or another, above the animal biosphere a human sphere, a sphere of reflection, of conscious invention, of conscious souls (the noosphere, if you will)"

DeLong, J.P., Okie, J.G., Moses, M.E., Sibly, R.M. and Brown, J.H., 2010. Shifts in metabolic scaling, production, and efficiency across major evolutionary transitions of life. Proceedings of the National Academy of Sciences, 107(29), pp.12941-12945.

Department of Energy, 2016, Lessons Learned Quarterly Report, December 2, 2016; Issue No. 89

Desroches, T.C., McMullin, D.R. and Miller, J.D., 2014. Extrolites of Wallemia sebi, a very common fungus in the built environment. Indoor air, 24(5), pp.533-542.

de Vera, J.P., Schulze-Makuch, D., Khan, A., Lorek, A., Koncz, A., Möhlmann, D. and Spohn, T., 2014. Adaptation of an Antarctic lichen to Martian niche conditions can occur within 34 days. Planetary and Space Science, 98, pp.182-190. See also summary Koh Xuan Yang, 2014, Adaptation of Antarctic Lichens to Conditions on Mars, Beyond Earthly Skies

Dhami, N.K., Reddy, M.S., Mukherjee, A., 2013. Biomineralization of calcium carbonates and their engineered applications: a review. Frontiers in microbiology, 4, p.314.

Doolittle W. F., 2000, Uprooting the Tree of Life, Scientific American

As Woese has written, "The ancestor cannot have been a particular organism, a single organismal lineage. It was communal, a loosely knit, diverse conglomeration of primitive cells that evolved as a unit, and it eventually developed to a stage where it broke into several distinct communities, which in their turn become the three primary lines of descent [bacteria, archaea and eukaryotes]." In other words, early cells, each having relatively few genes, differed in many ways. By swapping genes freely, they shared various of their talents with their contemporaries.

Eventually this collection of eclectic and changeable cells coalesced into the three basic domains known today. These domains remain recognizable because much 
(though by no means all) of the gene transfer that occurs these days goes on within domains.

Doyle, A., 2014, Mapping Amino Acids to Understand Life's Origins, Astrobiology Magazine

Drake, H., Åström, M.E., Heim, C., Broman, C., Åström, J., Whitehouse, M., Ivarsson, M., Siljeström, S. and Sjövall, P., 2015. Extreme $13 \mathrm{C}$ depletion of carbonates formed during oxidation of biogenic methane in fractured granite. Nature communications, 6, p.7020.

Dundas, C.M., McEwen, A.S., Chojnacki, M., Milazzo, M.P., Byrne, S., McElwaine, J.N. and Urso, A., 2017. Granular flows at recurring slope lineae on Mars indicate a limited role for liquid water. Nature Geoscience, 10(12), p.903.

Dunlop, R.A., Cox, P.A., Banack, S.A. and Rodgers, K.J., 2013. The non-protein amino acid BMAA is misincorporated into human proteins in place of L-serine causing protein misfolding and aggregation. PloS one, 8(9), p.e75376.

Edwards, C.S. and Piqueux, S., 2016. The water content of recurring slope lineae on Mars. Geophysical Research Letters, 43(17), pp.8912-8919. NASA press release: Test for Damp Ground at Mars' Seasonal Streaks Finds None

Ehlmann, B.L., Mustard, J.F., Swayze, G.A., Clark, R.N., Bishop, J.L., Poulet, F., Des Marais, D.J., Roach, L.H., Milliken, R.E., Wray, J.J. and Barnouin-Jha, O., 2009. Identification of hydrated silicate minerals on Mars using MRO-CRISM: Geologic context near Nili Fossae and implications for aqueous alteration. Journal of Geophysical Research: Planets, 114(E2).

Eugster, O., Busemann, H., Lorenzetti, S. and Terribilini, D., 2002. Ejection ages from krypton-81-krypton-83 dating and pre-atmospheric sizes of Martian meteorites. Meteoritics \& Planetary Science, 37(10), pp.1345-1360.

Fairén, A.G., Parro, V., Schulze-Makuch, D. and Whyte, L., 2017. Searching for life on Mars before it is too late. Astrobiology, 17(10), pp.962-970.

Fairén, A.G., Parro, V., Schulze-Makuch, D. and Whyte, L., 2018. Is Searching for Martian Life a Priority for the Mars Community?. Astrobiology, 18(2), pp.101-107.

Fastook, J.L., Head, J.W., Marchant, D.R., Forget, F. and Madeleine, J.B., 2012. Early Mars climate near the Noachian-Hesperian boundary: Independent evidence for cold conditions from basal melting of the south polar ice sheet (Dorsa Argentea Formation) and implications for valley network formation. Icarus, 219(1), pp.25-40. Summary and interview: Burnham, R., 2012, Southern polar eskers point to warmer past, Red Planet Report 
Fawdon, P., Skok, J.R., Balme, M.R., Vye-Brown, C.L., Rothery, D.A. and Jordan, C.J., 2015. The geological history of Nili Patera, Mars. Journal of Geophysical Research: Planets, 120(5), pp.951-977.

Fenner, F., 2010. Deliberate introduction of the European rabbit, Oryctolagus cuniculus, into Australia. Revue scientifique et technique, 29(1), p.103.

Fernanda Pires, 2015, "Mars liquid water: Curiosity confirms favorable conditions", Michigan news.

"Life as we know it needs liquid water to survive. While the new study interprets Curiosity's results to show that microorganisms from Earth would not be able to survive and replicate in the subsurface of Mars, Rennó sees the findings as inconclusive. He points to biofilms-colonies of tiny organisms that can make their own microenvironment."

Fischer, E., Martínez, G.M., Elliott, H.M. and Rennó, N.O., 2014. Experimental evidence for the formation of liquid saline water on Mars. Geophysical research letters, 41(13), pp.4456-4462. See also press release, Fischer, E., 2014, Martian salts must touch ice to make liquid water, study shows, American Geophysical Union.

Fishbaugh, K.E., Poulet, F., Chevrier, V., Langevin, Y. and Bibring, J.P., 2007. On the origin of gypsum in the Mars north polar region. Journal of Geophysical Research: Planets, 112(E7).

Fisk, M., Popa, R., Bridges, N., Renno, N., Mischna, M., Moores, J. and Wiens, R., 2013. Habitability of Transgressing Mars Dunes. Geochimica et Cosmochimica Acta, 67, pp.3871-3887.

Flahaut, J., Quantin, C., Clenet, H., Allemand, P., Mustard, J.F. and Thomas, P., 2012. Pristine Noachian crust and key geologic transitions in the lower walls of Valles Marineris: Insights into early igneous processes on Mars. Icarus, 221(1), pp.420-435.

Fries, M., Christou, A., Archer, D., Conrad, P., Cooke, W., Eigenbrode, J., ten Kate, I.L., Matney, M., Niles, P., Sykes, M. and Steele, A., 2016. A cometary origin for Martian atmospheric methane. Geochem. Perspect. Lett, 2, pp.10-23.

Fritz, J., Artemieva, N. and Greshake, A., 2005. Ejection of Martian meteorites. Meteoritics \& Planetary Science, 40(9-10), pp.1393-1411.

Galletta, G., Bertoloni, G. and D'Alessandro, M., 2010. Bacterial survival in martian conditions. arXiv preprint arXiv:1002.4077.

Gardin, E., Allemand, P., Quantin, C. and Thollot, P., 2010. Defrosting, dark flow features, and dune activity on Mars: Example in Russell crater. Journal of Geophysical Research:

Planets, 115(E6). 
Gaskin, J.A., Jerman, G., Gregory, D. and Sampson, A.R., 2012, March. Miniature variable pressure scanning electron microscope for in-situ imaging \& chemical analysis. In

Aerospace Conference, 2012 IEEE (pp. 1-10). IEEE.

Gladman, B.J., Burns, J.A., Duncan, M., Lee, P. and Levison, H.F., 1996. The exchange of impact ejecta between terrestrial planets. Science, 271(5254), pp.1387-1392.

Gladman, B., Dones, L., Levison, H.F. and Burns, J.A., 2005. Impact seeding and reseeding in the inner Solar System. Astrobiology, 5(4), pp.483-496.

Goetz, W., Brinckerhoff, W.B., Arevalo, R., Freissinet, C., Getty, S., Glavin, D.P., Siljeström, S., Buch, A., Stalport, F., Grubisic, A. and Li, X., 2016. MOMA: the challenge to search for organics and biosignatures on Mars. International Journal of Astrobiology, 15(3), pp.239-250.

Gordon, E., Mouz, N., Duee, E. and Dideberg, O., 2000. The crystal structure of the penicillin-binding protein $2 x$ from Streptococcus pneumoniae and its acyl-enzyme form: implication in drug resistance. Journal of molecular biology, 299(2), pp.477-485.

Greenberg, R. and Tufts, B.R., 2001. Macroscope: Infecting Other Worlds. American Scientist, 89(4), pp.296-299.

"As long as the probability of people infecting other planets with terrestrial microbes is substantially smaller than the probability that such contamination happens naturally, exploration activities would, in our view, be doing no harm. We call this concept the natural contamination standard."

Gronstal, A. L., 2014, "BIOMEX: Exploring Mars in Low Earth Orbit. Astrobiology Magazine (NASA).

Gros, C., 2016. Developing ecospheres on transiently habitable planets: the genesis project. Astrophysics and Space Science, 361(10), p.324.

Grant, J.A., Irwin III, R.P. and Wilson, S.A., 2010. Aqueous depositional settings in Holden crater, Mars. Lakes on Mars, pp.323-346. See also Grant, J., Irwin, R., Milliken, R., M2020 Candidate Landing Site Data Sheets - HOLDEN CRATER and Possible MSL Landing Site: Holden Crater

Haberle, R.M., McKay, C.P., Schaeffer, J., Cabrol, N.A., Grin, E.A., Zent, A.P. and Quinn, R., 2001. On the possibility of liquid water on present-day Mars. Journal of Geophysical Research: Planets, 106(E10), pp.23317-23326.

Hamilton, D.B., 2016. Biosignature detection in Mars analogue aqueous alteration minerals using Raman spectroscopy. 
Hand, K.P., Murray, A.E., Garvin, J.B., Brinckerhoff, W.B., Christner, B.C., Edgett, K.S., Ehlmann, B.L., German, C.R., Hayes, A.G., Hoehler, T.M., Horst, S.M., Lunine, J.I., Nealson, K.H., Paranicas, C., Schmidt, B.E., Smith, D.E., Rhoden, A.R., Russell, A.R., Russell, M.J., Templeton, A.S., Willis, P.A., Yingst, R.A., Phillips, C.B., Cable, M.L., Craft, K.L., Hofmann, A.E., Northeim, T.A., Pappalardo, R.P., and the Project Engineering Team (2017): Report of the Europa Lander Science Definition Team. February 2017.

Hartvigsen, J., Elangovan, S., Elwell, J. and Larsen, D., 2017. Oxygen Production from Mars Atmosphere Carbon Dioxide Using Solid Oxide Electrolysis. ECS Transactions, 78(1), pp.2953-2963.

Head, J.N., Melosh, H.J. and Ivanov, B.A., 2002. Martian meteorite launch: High-speed ejecta from small craters. Science, 298(5599), pp.1752-1756.

Nishiizumi et al. (1986) found that all cosmogenic nuclide data indicate that the shergottites were ejected from $>3 \mathrm{~m}$ depth. This conclusion was supported by Reedy (1989) stating that the Shergottite-Nakhdite-Chassignite group meteorites (SNCs), especially the shergottites, must have been buried $>5 \mathrm{~m}$ in any previous parent object (corresponding to a shielding depth of $>1500$ glcm2)

Hendrix, A.R. and Yung, Y.L., 2017. Energy Options for Future Humans on Titan. arXiv preprint arXiv:1707.00365.

Hoff, B., Thomson, G. and Graham, K., 2007. Ontario: Neurotoxic cyanobacterium (blue-green alga) toxicosis in Ontario. The Canadian Veterinary Journal, 48(2), p.147.

Hoffman N, Kyle PR, 2003,. The ice towers of Mt. Erebus as analogues of biological refuges on Mars. In Sixth International Conference on Mars 2003 Jul. Described in 2003, Martian Hot Spots,NASA Astrobiology Magazine.

Holson, D.A.. 2015, Ackee Fruit Toxicity, Medscape - Emergency medicine

Hopkins, J.B. and Pratt, W.D., 2011, September. Comparison of Deimos and Phobos as destinations for human exploration, and identification of preferred landing sites. In AIAA Space 2011 Conference \& Exposition, Long Beach (pp. 27-29).

Houtkooper, J.M. and Schulze-Makuch, D., 2006. A possible biogenic origin for hydrogen peroxide on Mars: the Viking results reinterpreted. arXiv preprint physics/0610093.

Houtkooper, J.M. and Schulze-Makuch, D., 2010. The possible role of perchlorates for Martian life. Journal of Cosmology, 5, pp.930-939.

Hu, R., Bloom, A.A., Gao, P., Miller, C.E. and Yung, Y.L., 2016. Hypotheses for near-surface exchange of methane on Mars. Astrobiology, 16(7), pp.539-550.

Hu, R., Kass, D.M., Ehlmann, B.L. and Yung, Y.L., 2015. Tracing the fate of carbon and the atmospheric evolution of Mars. Nature communications, 6, p.10003. 
Jheeta, S., 2013. Horizontal gene transfer and its part in the reorganisation of genetics during the LUCA epoch .Life (Basel), 3(4): 518-523.

"What are the mechanisms by which HGT occurs? Currently these include: transduction, a process whereby a viral capsule is used to transfer genetic material from one cell to another; conjugation, a process exhibited by microbes during which a plasmid or a small piece of a plasmid from one donor cell is transferred to another recipient cell (Prof. Matxalen Llosa-see summary report); transformation, which occurs when a competent cell takes up a "naked" strands of nucleic acid from the environment-such strands of nucleic acids may not necessarily have been exuded by living entities (e.g., mitochondrion genes transferred to eukaryote chromosomes), they could also be from recently dead cells, as well as from long extinct organisms; gene transfer agents (GTA), which are bacteriophage-like particles containing random cellular genomic segments intended for transduction to another living recipient cell; and membrane vesicle transfer (MVT), in which small membrane sacs emanating from the surface of a cell contain genetic material for transfer to another living recipient cell."

Johnson, R.D. and Holbrow, C.H. eds., 1977. Space settlements: A design study (Vol. 413). Scientific and Technical Information Office, National Aeronautics and Space Administration.

"At all distances out to the orbit of Pluto and beyond, it is possible to obtain Earth-normal solar intensity with a concentrating mirror whose mass is small compared to that of the habitat."

\section{chapter 7}

Johnson, S.S., Mischna, M.A., Grove, T.L. and Zuber, M.T., 2008. Sulfur-induced greenhouse warming on early Mars. Journal of Geophysical Research: Planets, 113(E8).

For instance 2.11 microbars of $\mathrm{H} 2 \mathrm{~S}$ would be sufficient for a $5 \mathrm{C}$ increase in temperature in a 50 mbar CO2 atmosphere (Table 3, combined with Table 2 for the partial pressures), Also, sulfur dioxide's effect is amplified by water vapour, especially in a dense atmosphere, while water vapour actually reduces the greenhouse effect for hydrogen sulfide.

Kahn, R., 1985. The evolution of CO2 on Mars. Icarus, 62(2), pp.175-190.

Kapoor, G., Saigal, S. and Elongavan, A., 2017. Action and resistance mechanisms of antibiotics: A guide for clinicians. Journal of anaesthesiology, clinical pharmacology, 33(3), p.300.

Kerber, L. and Nesnas, I.A., 2016, October. The Axel Rover: A Novel Platform for Instruments Making Measurements in Extreme Terrains. In 3rd International Workshop on Instrumentation for Planetary Mission (Vol. 1980).

Kerwick, T.B., 2012. Colonizing Jupiter's Moons: An Assessment of Our Options and Alternatives. Journal of the Washington Academy of Sciences, pp.15-26. 
Khayat, A., Villanueva, G.L., Mumma, M.J. and Tokunaga, A.T., 2014, November. A new search for active release of volcanic gases on Mars: Sensitive upper limits for OCS. In AAS/Division for Planetary Sciences Meeting Abstracts (Vol. 46). Also described by Howell, E., 2015, The Search For Volcanic Eruptions On Mars Reaches The Next Level, NASA Astrobiology Magazine.

Kite, E.S., Gaidos, E. and Onstott, T.C., 2018. Valuing life detection missions. arXiv preprint arXiv:1802.09006.

Kite, E.S., Mischna, M.A., Gao, P. and Yung, Y.L., 2017. Climate optimum on Mars initiated by atmospheric collapse. arXiv preprint arXiv:1709.08302.

Klingler, J.M., Mancinelli, R.L. and White, M.R., 1989. Biological nitrogen fixation under primordial Martian partial pressures of dinitrogen. Advances in Space Research, 9(6), pp.173-176.

Koonin, E.V., 2014. Carl Woese's vision of cellular evolution and the domains of life. RNA biology, 11(3), pp.197-204.

Kwong, J., Norris, S.D., Hopkins, J.B., Buxton, C.J., Pratt, W.D. and Jones, M.R., 2011, September. Stepping stones: exploring a series of increasingly challenging destinations on the way to mars. In AIAA Space 2011 Conference, Long Beach, CA (pp. 27-29).

Kvenvolden, K.A., Lawless, J.G. and Ponnamperuma, C., 1971. Nonprotein amino acids in the Murchison meteorite. Proceedings of the National Academy of Sciences, 68(2), pp.486-490.

Landis, G.A. and Haag, E., 2013. Analysis of Solar Cell Efficiency for Venus Atmosphere and Surface Missions. In 11th International Energy Conversion Engineering Conference (p. 4028).

Landis, G.A., Oleson, S.R. and Grantier, D., 2014. Zephyr: A Landsailing Rover for Venus, 65th International Astronautical Congress.

Lane, N., 2015. The vital question: energy, evolution, and the origins of complex life. WW Norton \& Company, page 49.

"Microbes are not equivalent to large animals: their population sizes are enormously larger, and they pass around useful genes (such as those for antibiotic resistance) by lateral transfer, making them very much less vulnerable to extinction. There is no hint of any microbial extinction even in the aftermath of the Great Oxygenation Event. The 'oxygen holocaust', which supposedly wiped out most anaerobic cells, can't be traced at all; there is no evidence from either phylogenetics or geochemistry that such an extinction ever took place. On the contrary, anaerobes prospered."

Lanza, N.L., Fischer, W.W., Wiens, R.C., Grotzinger, J., Ollila, A.M., Cousin, A., Anderson, R.B., Clark, B.C., Gellert, R., Mangold, N. and Maurice, S., 2014. High manganese 
concentrations in rocks at Gale crater, Mars. Geophysical Research Letters, 41(16), pp.5755-5763.

Popular exposition: Nina Lanza, How a weird Mars rock may be solid proof of an ancient oxygen atmosphere, Astronomy magazine

Lapen, T.J., Righter, M., Brandon, A.D., Debaille, V., Beard, B.L., Shafer, J.T. and Peslier, A.H., 2010. A younger age for ALH84001 and its geochemical link to shergottite sources in Mars. Science, 328(5976), pp.347-351.

Latgé, J.P., 1999. Aspergillus fumigatus and aspergillosis. Clinical microbiology reviews, 12(2), pp.310-350.

Lebeaux, D., Chauhan, A., Rendueles, O. and Beloin, C., 2013. From in vitro to in vivo models of bacterial biofilm-related infections. Pathogens, 2(2), pp.288-356.

Leber, A.L., 2016. Clinical microbiology procedures handbook. John Wiley \& Sons.

Lederberg, J. 1959, letter to J.B.S. Haldane

"Just as $i$ started to write this letter I realized there might have been a substantial connection between its import and the occasion of my visit to you November 6, $1957 \ldots$.

"It must have been around this time surely that I began to think of the scientific consequences of lunar and planetary probes. ... I have in mind the quite tangible possibility of contamination by terrestrial organisms of the surfaces of Mars and Venus, unless stringent precautions are taken to sterilize any vehicles sent there...."

Lederberg, J., 1999a. Paradoxes of the host-parasite relationship. ASM News, 65(12).

Lederberg, J., 1999b. Parasites face a perpetual dilemma. ASM News, 65(2).

Leflaive, J. and Ten-hage, L.O.Ï.C., 2007. Algal and cyanobacterial secondary metabolites in freshwaters: a comparison of allelopathic compounds and toxins. Freshwater Biology, 52(2), pp.199-214.

Leslie E, O., 2004. Prebiotic chemistry and the origin of the RNA world. Critical reviews in biochemistry and molecular biology, 39(2), pp.99-123.

"A scenario that I personally find attractive is one in which the very first replicators were 'naked genes' adsorbed on the surface of mineral particles, and in which impermeable membrane caps were 'invented' by the genetic system as it became metabolically competent. Escape from the mineral surface, enabled by the development of a closed spherical membrane would occur at a relatively late stage in evolution" 
Levin, G.V. and Straat, P.A., 2016. The case for extant life on Mars and its possible detection by the Viking labeled release experiment. Astrobiology, 16(10), pp.798-810.

Levy, M. and Miller, S.L., 1998. The stability of the RNA bases: implications for the origin of life. Proceedings of the National Academy of Sciences, 95(14), pp.7933-7938.

Lin, Y., El Goresy, A., Hu, S., Zhang, J., Gillet, P., Xu, Y., Hao, J., Miyahara, M., Ouyang, Z., Ohtani, E. and Xu, L., 2014. NanoSIMS analysis of organic carbon from the Tissint Martian meteorite: Evidence for the past existence of subsurface organic-bearing fluids on Mars. Meteoritics \& Planetary Science, 49(12), pp.2201-2218.

Lindensmith, C.A., Rider, S., Bedrossian, M., Wallace, J.K., Serabyn, E., Showalter, G.M., Deming, J.W. and Nadeau, J.L., 2016. A submersible, off-axis holographic microscope for detection of microbial motility and morphology in aqueous and icy environments. PloS one, 11(1), p.e0147700.

Lindquist, J., 2006, Bacteriology 102: Enrichment and Isolation of Purple Non-Sulfur Photosynthetic Bacteria

Losiak, A. and Velbel, M.A., 2011. Evaporite formation during weathering of Antarctic meteorites_A weathering census analysis based on the ANSMET database. Meteoritics \& Planetary Science, 46(3), pp.443-458.

Losiak, A., Czechowski, L. and Velbel, M.A., 2014, September. Ice Melting by Radiantly Heated Dust Grains on the Martian Northern Pole. In 77th Annual Meeting of the Meteoritical Society (Vol. 1800).

Lucchetti, A., Cremonese, G., Cambianica, P., Daubar, I., McEwen, A.S. and Re, C., 2015, December. Numerical Modelling and Ejecta Distribution Analysis of a Martian Fresh Crater. In AGU Fall Meeting Abstracts. Photograph released by JPL as A Spectacular New Martian Impact Crater (2014).

Lüthi, D., 2008. EPICA Dome C Ice Core 800KYr Carbon Dioxide Data. IGBP PAGES/World Data Center for Paleoclimatology Data Contribution Series, 55. Also, plotted as a graph by Robert Simmon for NASA Earth Observatory on the page Changes in the Carbon Cycle.

Mancinelli, R.L., 1993, personal communication with D. Thomas at NASA Ames Research center, cited in Thomas, D., 1995, Biological aspects of the ecopoesis and terraformation of Mars: Current perspectives and research, Journal of the British Interplanetary Society, vol 48, pp 415 - 418,

"Additional unpublished research revealed nitrogen fixation by a variety of microorganisms at $\mathrm{pN}$ of 0.2 mbar - the current partial pressure of nitrogen in the Mars atmosphere." 
Martel, L.M., 2001. If Lava Mingled with Ground Ice on Mars. Planetary Science Research Discoveries Report.

Martins, Z., Alexander, C.O.D., Orzechowska, G.E., Fogel, M.L. and Ehrenfreund, P., 2007. Indigenous amino acids in primitive CR meteorites. Meteoritics \& Planetary Science, 42(12), pp.2125-2136.

Martín-Torres, F.J., Zorzano, M.P., Valentín-Serrano, P., Harri, A.M., Genzer, M., Kemppinen, O., Rivera-Valentin, E.G., Jun, I., Wray, J., Madsen, M.B. and Goetz, W., 2015. Transient liquid water and water activity at Gale crater on Mars. Nature Geoscience, 8(5), p.357. Summary: "Evidence of liquid water found on Mars (BBC)

Martin, M.O., 2002. Predatory prokaryotes: an emerging research opportunity. Journal of molecular microbiology and Biotechnology, 4(5), pp.467-478.

Martínez, G.M. and Renno, N.O., 2013. Water and brines on Mars: current evidence and implications for MSL. Space Science Reviews, 175(1-4), pp.29-51. See particularly section 3.1.2 Dune Dark Spots and Flow-like Features

Massé, M., Bourgeois, O., Le Mouélic, S., Verpoorter, C., Spiga, A. and Le Deit, L., 2012. Wide distribution and glacial origin of polar gypsum on Mars. Earth and Planetary Science Letters, 317, pp.44-55.

Matthews, D., Jones, H., Gans, P., Coates, S. and Smith, L.M., 2005. Toxic secondary metabolite production in genetically modified potatoes in response to stress. Journal of Agricultural and Food Chemistry, 53(20), pp.7766-7776.

McGuire, M.L., Borowski, S.K., Mason, L.M. and Gilland, J., 2003. High power MPD nuclear electric propulsion (NEP) for artificial gravity HOPE missions to Callisto.

McEwen, A.S., Ojha, L., Dundas, C.M., Mattson, S.S., Byrne, S., Wray, J.J., Cull, S.C., Murchie, S.L., Thomas, N. and Gulick, V.C., 2011. Seasonal flows on warm Martian slopes. Science, 333(6043), pp.740-743.

McKay, C.P., 2009. Planetary ecosynthesis on Mars: restoration ecology and environmental ethics. Exploring the origin, extent, and future of life: Philosophical, ethical, and theological perspectives, pp.245-260.

McKay, C.P., Stoker, C.R., Glass, B.J., Davé, A.I., Davila, A.F., Heldmann, J.L., Marinova, M.M., Fairen, A.G., Quinn, R.C., Zacny, K.A. and Paulsen, G., 2013. The Icebreaker Life Mission to Mars: a search for biomolecular evidence for life, Astrobiology, 13(4), pp.334-353.

McLean, R.J. and McLean, M.A., 2010. Microbial Survival Mechanisms and the Interplanetary Transfer of Life Through Space. Journal of Cosmology, 7, pp.1802-1820. 
Mégarbane, B., Borron, S.W. and Baud, F.J., 2005. Current recommendations for treatment of severe toxic alcohol poisonings. Intensive care medicine, 31(2), pp.189-195.

Meltzer, M., 2007. Mission to Jupiter: a history of the Galileo project. NASA STI/Recon Technical Report N, 7.

Meltzer, M., 2012. When Biospheres Collide: A History of NASA's Planetary Protection Programs. Government Printing Office, After Splashdown: Plans To Safely Transport the Apollo Astronauts, Command Module, and Samples to the Recovery Ship, Page 217 and following

Meringer, M., Cleaves, H.J. and Freeland, S.J., 2013. Beyond terrestrial biology: Charting the chemical universe of $\alpha$-amino acid structures. Journal of chemical information and modeling, 53(11), pp.2851-2862.

Michalski, J.R. and Niles, P.B., 2010a. Deep crustal carbonate rocks exposed by meteor impact on Mars. Nature Geoscience, 3(11), p.751.

Michalski, J.R., Bibring, J.P., Poulet, F., Loizeau, D., Mangold, N., Dobrea, E.N., Bishop, J.L., Wray, J.J., McKeown, N.K., Parente, M. and Hauber, E., 2010b. The Mawrth Vallis region of Mars: A potential landing site for the Mars Science Laboratory (MSL) mission. Astrobiology, 10(7), pp.687-703. Short summary: Possible MSL Landing Site: Mawrth Vallis

Mileikowsky, C., Cucinotta, F.A., Wilson, J.W., Gladman, B., Horneck, G., Lindegren, L., Melosh, J., Rickman, H., Valtonen, M. and Zheng, J.Q., 2000. Natural transfer of viable microbes in space: 1. From Mars to Earth and Earth to Mars. Icarus, 145(2), pp.391-427.

P395 "For the temperature rise at ejection to be $<100 \mathrm{C}$, the maximum pressure, $\mathrm{P}$ max , must be less than $1 \mathrm{Gp"}$

Table 1 page

8.2 "The number of ejecta from Earth landing on Mars from 4.0 Ga to present time (Table II) was one to two orders of magnitude lower than the number of martian meteorites landing on Earth during the same period of time, but nevertheless still very substantial, about a billion during the last four billion years. How-ever, what fraction of the cells on board could survive? The principal differences between Earth-to-Mars transfers and Mars-to-Earth transfers are the following: - The escape velocity from Earth is $11 \mathrm{~km} / \mathrm{s}$, compared to $5 \mathrm{~km} / \mathrm{s}$ from Mars. —Of ejecta emitted from Earth, roughly $0.01 \%$ land on Mars, within about $1 \mathrm{Ma}$, whereas ${ }^{a} 0.5 \%$ of martian ejecta land on Earth, a difference of a factor of 50. - The natural radioactivity in meteorites ejected from Earth is usually much higher-by more than a factor of 10-than that in martian meteorites (Table V). - The difference in atmospheric pressure that has existed most of the time: for almost $4 \mathrm{Ga}$ Mars' surface-level atmospheric pressure has on average been less than the present 7 millibars. This is an advantage in terms of transfer from Mars to Earth, with low 
resistance and heating for the outgoing ejecta and gradual braking during the fall through Earth's progressively denser atmosphere. -Ejecta from Earth to Mars on the other hand do not enjoy such advantages. On their way out they immediately meet the resistance and heating of Earth's full atmospheric pressure, and on approaching Mars no significant atmosphere softens the hypervelocity landing."

Miller, J.D., Straat, P.A. and Levin, G.V., 2002, February. Periodic analysis of the Viking lander Labeled Release experiment. In Instruments, Methods, and Missions for Astrobiology IV (Vol. 4495, pp. 96-108). International Society for Optics and Photonics.

Möhlmann, D.T.F., 2009. Liquid interfacial and melt-water in the upper sub-surface of Mars. In Workshop on Modeling Martian Hydrous Environments (Vol. 1482, p. 48). Lunar and Planetary Institute.

Möhlmann, D., 2010a. The three types of liquid water in the surface of present Mars. International Journal of Astrobiology, 9(1), pp.45-49.

Möhlmann, D.T., 2010b. Temporary liquid water in upper snow/ice sub-surfaces on Mars?. Icarus, 207(1), pp.140-148.

Möhlmann, D.T., 2010c. Temporary liquid water in upper snow/ice sub-surfaces on Mars? Icarus, 207(1), pp.140-148, and as interviewed by Shiga, D., 2009, Watery niche may foster life on Mars, New Scientist

"Möhlmann's calculations assumed an impermeable upper crust of solid ice, which would form as water vapour diffused into pores and refroze. Such a seal would prevent evaporation and trap heat more effectively inside a snow bank, causing it to start melting in a zone that begins a few centimetres below the icy surface and extends a further 10 metres down, he says"

Mojarro, A., Hachey, J., Tani, J., Smith, A., Bhattaru, S., Pontefract, A., Doebler, R., Brown, M., Ruvkun, G., Zuber, M.T. and Carr, C.E., 2016, October. SETG: nucleic acid extraction and sequencing for in situ life detection on Mars. In 3rd International Workshop on Instrumentation for Planetary Mission (Vol. 1980).

Mullen, L., 2003, Alien Infection, NASA Astrobiology Magazine By - Aug 25, 2003

Murray, J.B., Muller, J.P., Neukum, G., Werner, S., Hauber, E., Markiewicz, W., Head, J.W., Foing, B.H., Page, D., Mitchell, K.L. and Portyankina, G., 2005. Evidence from HRSC Mars Express for a frozen sea close to Mars' equator. Lunar and Planetary Science XXXVI, 1234, pp.CD-ROM. See also Murray, L., 2005, The Martian Frozen Sea - Interview with John Murray, NASA Astrobiology Magazine 
Nadeau, J., Lindensmith, C., Deming, J.W., Fernandez, V.I. and Stocker, R., 2016.

Microbial morphology and motility as biosignatures for outer planet missions. Astrobiology, 16(10), pp.755-774.

NASA press release, 2017, NASA Tests Robotic Ice Tools

NASA video, 2017, A guide to Gale crater

Naseem, M., Osmanoglu, Ö. and Dandekar, T., 2020. Synthetic Rewiring of Plant CO2 Sequestration Galvanizes Plant Biomass Production. Trends in Biotechnology, 38(4), pp.354-359.

The CETCH cycle requires less energy to operate than other aerobic $\mathrm{CO}_{2}$-fixation pathways. One limitation of $C E T C H$ is the production of glyoxylate, a less active metabolic intermediate that requires acetyl-CoA (AcCoA) or propanoyl-CoA [3] for conversion into other metabolites. Also, glyoxylate is not well connected to other metabolic pathways. Despite functional impediments associated with any synthetically designed pathway, CETCH is the most efficient artificial cycle that fixes (in vitro) several-fold more $\mathrm{CO}_{2}$ than does the natural $\mathrm{CBB}$. The incorporation of CETCH-based enoyl-CoA carboxylase/reductases (ECRs) should be an excellent alternative to the native Calvin cycle. It can sequester approximately $80 \mathrm{CO}_{2}$ molecules per second (in vitro) compared with RuBisCO, which fixes two to five $\mathrm{CO}_{2}$ molecules per second in plants.

National Research Council. 2009. Assessment of Planetary Protection Requirements for Mars Sample Return Missions (Report). p. 59.

"It has been estimated that the planning, design, site selection, environmental reviews, approvals, construction, commissioning, and pre-testing of a proposed SRF will occur 7 to 10 years before actual operations begin. In addition, 5 to 6 years will likely be required for refinement and maturation of SRF-associated technologies for safely containing and handling samples to avoid contamination and to further develop and refine biohazard-test protocols. Many of the capabilities and technologies will either be entirely new or will be required to meet the unusual challenges of integration into an overall (end-to-end) Mars sample return program."

National Safety Council, 2017 NSC Releases Annual Compilation of the Leading Causes of Preventable Death

Neukum, G., Jaumann, R., Hoffmann, H., Hauber, E., Head, J.W., Basilevsky, A.T., Ivanov, B.A., Werner, S.C., Van Gasselt, S., Murray, J.B. and McCord, T., 2004. Recent and episodic volcanic and glacial activity on Mars revealed by the High Resolution Stereo Camera. Nature, 432(7020), p.971.

Nicholson, W.L., 2009. Ancient micronauts: interplanetary transport of microbes by cosmic impacts. Trends in microbiology, 17(6), pp.243-250. 
Niles, P.B., Boynton, W.V., Hoffman, J.H., Ming, D.W. and Hamara, D., 2010. Stable Isotope Measurements of Martian Atmospheric CO2 at the Phoenix Landing Site. science, 329(5997), pp.1334-1337.

Nisbet, E., Zahnle, K., Gerasimov, M.V., Helbert, J., Jaumann, R., Hofmann, B.A., Benzerara, K. and Westall, F., 2007. Creating habitable zones, at all scales, from planets to mud micro-habitats, on Earth and on Mars. Space science reviews, 129(1-3), pp.79-121.

Noell, A.C., Fisher, A.M., Takano, N., Fors-Francis, K., Sherrit, S. and Grunthaner, F., 2016, October. Astrobionibbler: In Situ Microfluidic Subcritical Water Extraction of Amino Acids. In 3rd International Workshop on Instrumentation for Planetary Mission (Vol. 1980).

Nolan, K., 2008. Mars: A cosmic stepping stone. In MARS A Cosmic Stepping Stone (pp. 105-115). Springer, New York, NY. For the triple point feedback suggestion see page 137. Nott, J., 2009. Titan: a distant but enticing destination for human visitors. Aviation, space, and environmental medicine, 80(10), pp.900-901.

Oehler, D.Z. and Etiope, G., 2017. Methane seepage on Mars: where to look and why. Astrobiology, 17(12), pp.1233-1264.

Ojha, L., Wilhelm, M.B., Murchie, S.L., McEwen, A.S., Wray, J.J., Hanley, J., Massé, M. and Chojnacki, M., 2015. Spectral evidence for hydrated salts in recurring slope lineae on Mars. Nature Geoscience, 8(11), p.829.

Oleson, S.R., Landis, G.A., McGuire, M.L. and Schmidt, G.R., 2013. HERRO mission to Mars using telerobotic surface exploration from orbit

O'Malley-James, J.T., Greaves, J.S., Raven, J.A. and Cockell, C.S., 2013. Swansong biospheres: refuges for life and novel microbial biospheres on terrestrial planets near the end of their habitable lifetimes. International Journal of Astrobiology, 12(2), pp.99-112.

O'Malley-James, J.T., Cockell, C.S., Greaves, J.S. and Raven, J.A., 2014. Swansong biospheres II: The final signs of life on terrestrial planets near the end of their habitable lifetimes. International Journal of Astrobiology, 13(3), pp.229-243.

O'Malley-James, J.T., 2014. Life at the end of worlds: modelling the biosignatures of microbial life in diverse environments at the end of the habitable lifetimes of Earth-like planets (Doctoral dissertation, University of St Andrews).

Oren, A., Bardavid, R.E. and Mana, L., 2014. Perchlorate and halophilic prokaryotes: implications for possible halophilic life on Mars. Extremophiles, 18(1), pp.75-80.

Orosei, R., Lauro, S.E., Pettinelli, E., Cicchetti, A., Coradini, M., Cosciotti, B., Di Paolo, F., Flamini, E., Mattei, E., Pajola, M. and Soldovieri, F., 2018. Radar evidence of subglacial liquid water on Mars. Science, 361(6401), pp.490-493. 
Osman, S., Peeters, Z., La Duc, M.T., Mancinelli, R., Ehrenfreund, P. and Venkateswaran, K., 2008. Effect of shadowing on survival of bacteria under conditions simulating the Martian atmosphere and UV radiation. Applied and Environmental Microbiology, 74(4), pp.959-970.

Paige, D.A., 2000, July. Mars exploration strategies: Forget about sample return. In Concepts and Approaches for Mars Exploration (p. 243).

Parfrey, L.W., Lahr, D.J., Knoll, A.H. and Katz, L.A., 2011. Estimating the timing of early eukaryotic diversification with multigene molecular clocks. Proceedings of the National Academy of Sciences, 108(33), pp.13624-13629.

Parro, V., de Diego-Castilla, G., Moreno-Paz, M., Blanco, Y., Cruz-Gil, P., Rodríguez-Manfredi, J.A., Fernández-Remolar, D., Gómez, F., Gómez, M.J., Rivas, L.A. and Demergasso, C., 2011. A microbial oasis in the hypersaline Atacama subsurface discovered by a life detector chip: implications for the search for life on Mars. Astrobiology, 11(10), pp.969-996.

Pasini, D., 2014, April. Panspermia Survival Scenarios for Organisms that Survive Typical Hypervelocity Solar System Impact Events. In European Planetary Science Congress (Vol. 9).

Paulsen, G.L., Farritor, S., Huntsberger, T.L. and Aghazarian, H., 2005, April. All terrain exploration with the cliff-bot system. In Robotics and Automation, 2005. ICRA 2005. Proceedings of the 2005 IEEE International Conference on (pp. 721-726). IEEE.

Pavlov, A.K., Kalinin, V.L., Konstantinov, A.N., Shelegedin, V.N. and Pavlov, A.A., 2006. Was Earth ever infected by martian biota? Clues from radioresistant bacteria. Astrobiology, 6(6), pp.911-918

Perkel, J., 2016, Superresolution microscopy, Science/AAAS Custom Publishing Office

Pires, P. and Winter, O.C., 2020. Location and stability of Distant Retrograde Orbits around the Moon. Monthly Notices of the Royal Astronomical Society, 494(2), pp.2727-2735.

Pitt, J.I. and Christian, J.H.B., 1968. Water relations of xerophilic fungi isolated from prunes. Applied Microbiology, 16(12), pp.1853-1858.

Pommerol, A., Appéré, T., Portyankina, G., Aye, K.M., Thomas, N. and Hansen, C.J., 2013. Observations of the northern seasonal polar cap on Mars III: CRISM/HiRISE observations of spring sublimation. Icarus, 225(2), pp.911-922.

Pondrelli, M., Rossi, A.P., Marinangeli, L., Hauber, E., Gwinner, K., Baliva, A. and Di Lorenzo, S., 2008. Evolution and depositional environments of the Eberswalde fan delta, Mars. Icarus, 197(2), pp.429-451. Curiosity landing site selection summary: on Possible MSL Landing Site: Eberswalde Crater 
Quinn, R.C., Martucci, H.F., Miller, S.R., Bryson, C.E., Grunthaner, F.J. and Grunthaner, P.J., 2013. Perchlorate radiolysis on Mars and the origin of martian soil reactivity.

Astrobiology, 13(6), pp.515-520.

Pugel, D.B., Rummel, J.D. and Conley, C., 2017, March. Brushing your spacecraft's teeth: A review of biological reduction processes for planetary protection missions

. In Aerospace Conference, 2017 IEEE (pp. 1-10). IEEE.

Race, M. S., 1996, Planetary Protection, Legal Ambiguity, and the Decision Making Process for Mars Sample Return Adv. Space Res. vol 18 no 1/2 pp (1/2)345-(1/2)350

Race, M. R., Johnson, J.E., Spry, J.A., Siegel, B., Conley, C., 2015, Planetary Protection Knowledge Gaps for Human Extraterrestrial Missions Workshop Report, NASA Ames Research Center

- "Obviously, the current understanding of microbe survival in Mars dust environments remains uncertain and represents an important knowledge gap" (page 34)

Raffensperger, C., 1998, The Wingspread Consensus Statement on the Precautionary Principle

Reichhardt, T., 2016, Mars Needs Broadband!, Air and Space Magazine

Rettberg, P., Anesio, A.M., Baker, V.R., Baross, J.A., Cady, S.L., Detsis, E., Foreman, C.M., Hauber, E., Ori, G.G., Pearce, D.A. and Renno, N.O., 2016. Planetary protection and Mars special regions - a suggestion for updating the Idefinition.

Rummel, J.D., Beaty, D.W., Jones, M.A., Bakermans, C., Barlow, N.G., Boston, P.J., Chevrier, V.F., Clark, B.C., de Vera, J.P.P., Gough, R.V. and Hallsworth, J.E., 2014. A new analysis of Mars "special regions": findings of the second MEPAG Special Regions Science Analysis Group (SR-SAG2)..

Rummel, J. D., Conley C. A, 2017,.Four fallacies and an oversight: searching for martian life Astrobiology, 17(10), pp. 971-974.

Rummel, J.D. and Conley, C.A., 2018. Inadvertently Finding Earth Contamination on Mars Should Not Be a Priority for Anyone. Astrobiology, 18(2), pp.108-115.

Sagan, C. and Coleman, S., 1966. Decontamination Standards for Mars Exploration Programs. Biology and the Exploration of Mars, Space Science Board, National Academy of Sciences, Washington, DC, pp.470-481.

Sagan, C., 1973, The Cosmic Connection - an Extraterrestrial Perspective

Sagan, C., Levinthal, E.C. and Lederberg, J., 1968. Contamination of Mars. Science, 159(3820), pp.1191-1196. 
"The prominent dust storms and high wind velocities previously referred to imply that aerial transport of contaminants will occur on Mars. While it is probably true that a single unshielded terrestrial microorganism on the Martian surface ... would rapidly be enervated and killed by the ultraviolet flux, ... The Martian surface material certainly contains a substantial fraction of ferric oxides, which are extremely strongly absorbing in the near ultraviolet. ... A terrestrial microorganism imbedded in such a particle can be shielded from ultraviolet light and still be transported about the planet."

Sagan, C. Organic matter and the Moon., 1961, National Academy of Sciences.

Sakimoto, K.K., Wong, A.B. and Yang, P., 2016. Self-photosensitization of nonphotosynthetic bacteria for solar-to-chemical production. Science, 351(6268), pp.74-77.

Sakon, J.J. and Burnap, R.L., 2005, March. A Further Analysis of Potential Photosynthetic Life on Mars. In 36th Annual Lunar and Planetary Science Conference (Vol. 36).

Sakon, J.J. and Burnap, R.L., 2006. An analysis of potential photosynthetic life on Mars. International Journal of Astrobiology, 5(2), pp.171-180.

While isolating psychrophilic strains would likely provide a better analog for the Martian surface, the generation times are prohibitively slow for research purposes in such exploratory experiments

Salisbury, F.B., Gitelson, J.I. and Lisovsky, G.M., 1997. Bios-3: Siberian experiments in bioregenerative life support. BioScience, 47(9), pp.575-585.

Sarmiento, F., Peralta, R. and Blamey, J.M., 2015. Cold and hot extremozymes: industrial relevance and current trends. Frontiers in bioengineering and biotechnology, 3, p.148.

Sauder, J., Hilgemann, E., Johnson, M., Parness, A., Hall, J., Kawata, J. and Stack, K., 2017. Automation Rover for Extreme Environments.

We propose a novel approach that enables a long duration Venus rover mission by pairing clever, robust mechanisms with high temperature electronics that exist today. Electronic complexity is reduced by implementing mechanical analog systems in areas where electronics fall short. The final system is one in which the best of both high temperature electronics and mechanical systems are combined to make a system more capable than either technology on its own. Although the concept does not require significant advances in high temperature electronics, any developments in that area enhance the proposed concept.

An automaton rover could be subjected to much higher contamination control procedures than traditional rovers. It could be baked at extremely high temperatures, irradiated, and subjected to multiple chemical baths to kill any 
bacteria. Thus, automatons would be valuable in highly contamination control sensitive environments, like collecting samples from the dark, water streaks on Mars. In this type of situation, the automaton would likely be working in tandem with a traditional Mars rover

Scanlon, K.E., Head, J.W., Wilson, L. and Marchant, D.R., 2014. Volcano-ice interactions in the Arsia Mons tropical mountain glacier deposits. Icarus, 237, pp.315-339. Press release:

Stacey, K., 2014, A habitable environment on Martian volcano?, Brown university.

Scharf, C., 2016, How the Cold War Created Astrobiology, Life, death, and Sputnik, Nautilus Magazine.

Schirber, M.,, 2013, Searching for Organics in a Nibble of Soil NASA Astrobiology Magazine

Schirrmeister, B.E., Antonelli, A. and Bagheri, H.C., 2011. The origin of multicellularity in cyanobacteria. BMC evolutionary biology, 11(1), p.45.

Schlaepfer, M.A., Sax, D.F. and Olden, J.D., 2011. The potential conservation value of non-native species. Conservation Biology, 25(3), pp.428-437.

Schulze-Makuch, D. and Houtkooper, J.M., 2010. A perchlorate strategy for extreme xerophilic life on Mars. EPSC Abstracts, 5, pp.EPSC2010-308.

Schulze-Makuch, D., 2015, Sample Return May Not Be the Best Way to Find Martian Life, airspacemag.com

Moderator:, Schulze-Makuch, D., Participants:, Rummel, J.D., Benner, S.A., Levin, G., Parro, V. and Kounaves, S., 2015. Nearly Forty Years after Viking: Are We Ready for a New Life-Detection Mission?. Astrobiology, 15(6), pp.413-419. also summarized in Schulze-Makuch, D., 2015, The Right Time to Search for Martian Life, airspacemag.com

Schmidt, M.E., Ruff, S.W., McCoy, T.J., Farrand, W.H., Johnson, J.R., Gellert, R., Ming, D.W., Morris, R.V., Cabrol, N., Lewis, K.W. and Schroeder, C., 2008. Hydrothermal origin of halogens at Home Plate, Gusev crater. Journal of Geophysical Research: Planets, 113(E6).

Schwander, T., von Borzyskowski, L.S., Burgener, S., Cortina, N.S. and Erb, T.J., 2016. A synthetic pathway for the fixation of carbon dioxide in vitro. Science, 354(6314), pp.900-904.

Sella, S.R., Vandenberghe, L.P. and Soccol, C.R., 2014. Life cycle and spore resistance of spore-forming Bacillus atrophaeus. Microbiological research, 169(12), pp.931-939.

Seneviratne, G. and Indrasena, I.K., 2006. Nitrogen fixation in lichens is important for improved rock weathering. Journal of biosciences, 31(5), pp.639-643. 
Sharov, A.A., 2006. Genome increase as a clock for the origin and evolution of life. Biology Direct, 1(1), pp.1-10.

Shiga, D., 2009, First liquid water may have been spotted on Mars, New Scientist

Shirley, J.H., 2015. Solar System dynamics and global-scale dust storms on Mars. Icarus, 251, pp.128-144.

Skok, J.R., Mustard, J.F., Ehlmann, B.L., Milliken, R.E. and Murchie, S.L., 2010. Silica deposits in the Nili Patera caldera on the Syrtis Major volcanic complex on Mars. Nature Geoscience, 3(12), p.838.

Smith, M., 2016, Watzin to MEPAG: No 2022 Orbiter, No Decision on Helicopter, Space Policy Online.

Spaulding, S.A., Kilroy, C.A.T.H.Y. and Edlund, M.B., 2010. Diatoms as non-native species. The diatoms: applications for the environmental and earth sciences, pp.560-569.

Staehle, R. L., Fine,S. A. , Roberts, A., Schulenburg, C. R., and Skinner, D. L. 1976, Mars polar ice sample return mission ,Space-flight, 18, 399-409. and Space-flight, 19, 441-445, described in Portree, D.S.F., 2013 Mars Polar Ice Sample Return (1976-1978), Wired Science

Staehle, R.L., Spangelo, S., Lane, M.S., Aaron, K.M., Bhartia, R., Boland, J.S., Christensen, L.E., Forouhar, S., de la Torre Juarez, M., Trawny, N. and Webster, C.R., 2015. Multiplying Mars lander opportunities with MARSdrop microlanders. 29th Annual AIAA/USU Conference on Small Satellites

Stamenković, V., Ward, L. M., Mischna. M., Fischer. W. W.. "을 2 solubility in Martian near-surface environments and implications for aerobic life" - Nature, October 22, 2018 see also Vlada Stamenkovic. "Origins of Life \& Habitability - authors website with bibliography - and author shared link to the article", sharing is via Nature Sharedit — Habilabs

Stamenković, V., Sponges on Mars? We ask Stamenković about their oxygen-rich briny seeps model, 2019, personal communication - interview for WikiNews (extended version)

Stano, P. and Luisi, P.L., 2010. Chemical Approaches to Synthetic Biology-From Vesicles Self-Reproduction to Semi-Synthetic Minimal Cells. In ALIFE (pp. 147-153).

Stern, J.C., Sutter, B., Freissinet, C., Navarro-González, R., McKay, C.P., Archer, P.D., Buch, A., Brunner, A.E., Coll, P., Eigenbrode, J.L. and Fairen, A.G., 2015. Evidence for indigenous nitrogen in sedimentary and aeolian deposits from the Curiosity rover investigations at Gale crater, Mars. Proceedings of the National Academy of Sciences, 112(14), pp.4245-4250.

See also NASA press release: $\underline{\text { Curiosity Rover Finds Biologically Useful Nitrogen on Mars }}$ 
Stevenson, A., Burkhardt, J., Cockell, C.S., Cray, J.A., Dijksterhuis, J., Fox-Powell, M., Kee, T.P., Kminek, G., McGenity, T.J., Timmis, K.N. and Timson, D.J., 2015.Multiplication of microbes below 0.690 water activity: implications for terrestrial and extraterrestrial life. Environmental microbiology, 17(2), pp.257-277.

Stillman, D.E., Michaels, T.I. and Grimm, R.E., 2017. Characteristics of the numerous and widespread recurring slope lineae (RSL) in Valles Marineris, Mars. Icarus, 285, pp.195-210.

Stöffler, D., Horneck, G., Ott, S., Hornemann, U., Cockell, C.S., Moeller, R., Meyer, C., de Vera, J.P., Fritz, J. and Artemieva, N.A., 2007. Experimental evidence for the potential impact ejection of viable microorganisms from Mars and Mars-like planets. Icarus, 186(2), pp.585-588.

Strange, N., Landau, D., McElrath, T., Lantoine, G. and Lam, T., 2013. Overview of mission design for NASA asteroid redirect robotic mission concept.

Conley, C (2016). interviewed by Straus, M., for National Geographic, Going to Mars Could Mess Up the Hunt for Alien Life. Available at:

https://www.nationalgeographic.com/news/2016/09/mars-journey-nasa-alien-life-protection-h umans-planets-space/ (accessed 1 July 2020)

From the perspective of planetary protection, Conley is also concerned about terrestrial organisms that can absorb water from the air. She recalls fieldwork she did in the Atacama Desert in Chile, which is one of the driest places on Earth, with less than 0.04 inch of rain a year.

Even in this dessicated place, she found life: photosynthetic bacteria that had made a home in tiny chambers within halite salt crystals. There's a small amount of water retained inside the halite and, at night, it cools down and condenses both on the walls of the chambers and on the surface of the organisms that are sitting there.

Conley also warns that water contaminated with Earth microbes could pose serious problems if astronauts ever establish a base on Mars. Most current plans call for expeditions that rely on indigenous resources to sustain astronauts and reduce the supplies they would need to haul from Earth.

What if, for example, an advance mission carried certain types of bacteria known to create calcite when exposed to water? If such bacteria could survive on Mars, Conley says, future explorers prospecting for liquid water instead might find that underground aquifers have been turned into cement.

Stroupe, A., Huntsberger, A., Garrett, M. and Younse, P., 2007. Robotic Mars geology with TRESSA: beyond the Mars Rovers. In Workshop on Robotics and Challenging Environments, ICRA. 
Szostack, J., 2016, “On the Origin of Life", MEDICINA (BuenosAires) 2016; 76, 199-203 and Szostack lab summary

Tebo, B.M., Davis, R.E., Anitori, R.P., Connell, L.B., Schiffman, P. and Staudigel, H., 2015. Microbial communities in dark oligotrophic volcanic ice cave ecosystems of Mt. Erebus,

Antarctica. Frontiers in microbiology, 6, p.179.

Hoffman and Kyle suggested the ice towers of Mt. Erebus as analogues of biological refuges on Mars. They combined the idea of still existing near surface ice deposits with the assumption that there is still some localized volcanic activity on Mars today.

There are several examples from Mars that show a direct interaction between lava and ice in the geological history of Mars. The most obvious cases are the rootless cones seen in the northern lowlands. HRSC images show direct and violent interaction in the relatively recent geological history, for example at the scarps of Olympus Mons. Mars today is in relatively dormant phase, and any interactions which might be occurring today are presumably on a much less dynamic scale.

Nevertheless, they may be driving local hydrothermal systems. Studying the geothermal processes in the first few tens to hundreds of meters below the surface of Mars today might thus uncover a wide variety of new habitats where biological activity may survive on this cold and dry planet.

Thomet, L. and Sheridan, K., 2016, "Elon Musk envisions 'fun' but dangerous trips to Mars", Associated Free Press.

Trinks, H., Schröder, W. and Biebricher, C.K., 2005. Ice and the origin of life. Origins of Life and Evolution of Biospheres, 35(5), pp.429-445.

United Nations, 1966, resolution 2222 (XXI), Treaty on Principles Governing the Activities of States in the Exploration and Use of Outer Space, including the Moon and Other Celestial Bodies "Article IX: ... States Parties to the Treaty shall pursue studies of outer space, including the Moon and other celestial bodies, and conduct exploration of them so as to avoid their harmful contamination and also adverse changes in the environment of the Earth resulting from the introduction of extraterrestrial matter and, where necessary, shall adopt appropriate measures for this purpose..."

Valtonen, M., Nurmi, P., Zheng, J.Q., Cucinotta, F.A., Wilson, J.W., Horneck, G., Lindegren, L., Melosh, J., Rickman, H. and Mileikowsky, C., 2008. Natural transfer of viable microbes in space from planets in extra-solar systems to a planet in our solar system and vice versa.

The Astrophysical Journal, 690(1), p.210.

From our discussion above, it is clear that exchanges of bacteria between planets in different solar systems are only possible during the birth cluster stage of the systems in question. As the number of life-carrying bodies received by the Earth may have been in thousands, so also other planets in other stellar systems may have received their life from other members of our original star cluster, or even from a single source, the Earth.Thus the limited form of lithopanspermia inside a star cluster is possible, while the stronger version of life spreading through the whole Galaxy from a single source could not happen via mechanisms described in this work. But life-carrying 
bodies originating from our solar system may have found their way to our original neighbours, and that all conditions being optimal, life seeded by our system could have spread to many other solar systems. Here in our solar system our common ancestor cell most probably originated either on the Earth or on Mars. We cannot say for sure which one since there has been millions of potentially life-carrying transfers between these two planets. The GAIA mission will perhaps be able to locate the members of the birth cluster of the Sun while the SIM and DARWIN missions will be able to detect planets around them and search for signs of life in the planets. Even before these missions, the currently ongoing search for life in Mars may already give an indication how likely it is that life is transported between planets by natural means.

van Heereveld, L., Merrison, J., Nørnberg, P. and Finster, K., 2017. Assessment of the Forward Contamination Risk of Mars by Clean Room Isolates from Space-Craft Assembly Facilities through Aeolian Transport-a Model Study. Origins of Life and Evolution of Biospheres, 47(2), pp.203-21

Vartoukian, S.R., Palmer, R.M. and Wade, W.G., 2010. Strategies for culture of 'unculturable'bacteria. FEMS microbiology letters, 309(1), pp.1-7.

Velicer, G.J., Mendes-Soares, H.,2009, Bacterial Predators (quick guide), Current Biology Volume 19, Issue 2, Pages R55-R56

Verseux, C., Baqué, M., Cifariello, R., Fagliarone, C., Raguse, M., Moeller, R. and Billi, D., 2017. Evaluation of the resistance of Chroococcidiopsis spp. to sparsely and densely ionizing irradiation. Astrobiology, 17(2), pp.118-125.

Vítek, P., Edwards, H.G.M., Jehlička, J., Ascaso, C., De los Ríos, A., Valea, S., Jorge-Villar, S.E., Davila, A.F. and Wierzchos, J., 2010. Microbial colonization of halite from the hyper-arid Atacama Desert studied by Raman spectroscopy. Philosophical Transactions of the Royal Society of London A: Mathematical, Physical and Engineering Sciences, 368(1922), pp.3205-3221.

Wadsworth, J. and Cockell, C.S., 2017. Perchlorates on Mars enhance the bacteriocidal effects of UV light. Scientific reports, 7(1), p.4662. Interview: Daley, J. 2017, Mars Surface May Be Too Toxic for Microbial Life Smithsonian magazine

"The bacterial model we tested wasn't an extremophile so it's not out of the question that hardier life forms would find a way to survive."

Warmflash, D., Larios-Sanz, M., Jones, J., Fox, G.E. and McKay, D.S., 2007. Assessing the Biohazard Potential of Putative Martian Organisms for Exploration Class Human Space Missions.

"In essence, all that a potentially infectious human pathogen needs in order to emerge and persist in an environment is to grow and live naturally under conditions that are similar to those that it might later encounter in a human host. On Mars, these conditions might be met in a particular niche within the 
extracellular environment of a biofilm, or within the intracellular environment of another single-celled Martian organism."

Weiss, B.P., Kirschvink, J.L., Baudenbacher, F.J., Vali, H., Peters, N.T., Macdonald, F.A. and Wikswo, J.P., 2000. A low temperature transfer of ALH84001 from Mars to Earth.

Science, 290(5492), pp.791-795.

Weiss, I.M., Muth, C., Drumm, R. and Kirchner, H.O., 2018. Thermal decomposition of the amino acids glycine, cysteine, aspartic acid, asparagine, glutamic acid, glutamine, arginine and histidine. BMC biophysics, 11(1), p.2. For the decomposition temperatures see Table 1

Wells, L.E., Armstrong, J.C. and Gonzalez, G., 2003. Reseeding of early Earth by impacts of returning ejecta during the late heavy bombardment. Icarus, 162(1), pp.38-46.

"Conservatively evaluating experiments performed [by others] on Bacillus subtilis and Deinococcus radiodurans to constrain biological survival under impact conditions, we estimate that if the Earth were hit by a sterilizing impactor $~ 300 \mathrm{~km}$ in diameter, with a relative velocity of $30 \mathrm{~km} \mathrm{~s}^{-1}$ (such as may have occurred during the Late Heavy Bombardment), an initial cell population in the ejecta of order $10^{3}-10^{5}$ cells kg-1 would in most cases be sufficient for a single modern organism to survive and return to an again-clement planet $3000-5000$ years later. "

Westall, F., Loizeau, D., Foucher, F., Bost, N., Betrand, M., Vago, J. and Kminek, G., 2013. Habitability on Mars from a microbial point of view. Astrobiology, 13(9), pp.887-897.

Westall, F., Foucher, F., Bost, N., Bertrand, M., Loizeau, D., Vago, J.L., Kminek, G., Gaboyer, F., Campbell, K.A., Bréheret, J.G. and Gautret, P., 2015. Biosignatures on Mars: what, where, and how? Implications for the search for martian life. Astrobiology, 15(11), pp.998-1029.

Thus, whatever the scenario for the development of habitability, the relatively short period favorable for the appearance of life-early in the history of Mars (pre-Noachian to Noachian periods) - and the rapid discontinuity of habitable surface conditions (cf. Cockell et al., 2012) imply that, if life appeared on the Red Planet, it probably remained in a very primitive state of evolution, in most cases probably not achieving (anaerobic) photosynthesis.

Whitehead, R.A., Puil, E., Ries, C.R., Schwarz, S.K.W., Wall, R.A., Cooke, J.E., Putrenko, I., Sallam, N.A. and MacLeod, B.A., 2012. GABAB receptor-mediated selective peripheral analgesia by the non-proteinogenic amino acid, isovaline. Neuroscience, 213, pp.154-160.

Wierzchos, J., Davila, A.F., Sánchez-Almazo, I.M., Hajnos, M., Swieboda, R. and Ascaso, C., 2012. Novel water source for endolithic life in the hyperarid core of the Atacama Desert. Biogeosciences, 9(6), p.2275.

Wilcox, B.H., Carlton, J.A., Jenkins, J.M. and Porter, F.A., 2017, March. A deep subsurface ice probe for Europa. In Aerospace Conference, 2017 IEEE (pp. 1-13). IEEE. 
Witze, A., 2018. There's water on Mars! Signs of buried lake tantalize scientists. Nature, 560(7716), pp.13-15.

Woese, C., 1998. The universal ancestor. Proceedings of the national academy of Sciences, 95(12), pp.6854-6859.

There are different ways of looking at such a community of progenotes. On the one hand, it could have been the loose-knit evolutionary (genetic) community just discussed. On the other, it could have been more like a modern bacterial consortium, with cells cross-feeding one another not only genetically but also metabolically. Cell-cell contacts would have facilitated both processes. In both views of the community, the latter in particular, it is not individual cell lines but the community of progenotes as a whole that survives and evolves. It was such a community of progenotes, not any specific organism, any single lineage, that was our universal ancestor-a genetically rich, distributed, communal ancestor.

Woese, C.R., 2002. On the evolution of cells. Proceedings of the National Academy of Sciences, 99(13), pp.8742-8747.

"Aboriginal cell designs are taken to be simple and loosely organized enough that all cellular componentry can be altered and/or displaced through HGT, making HGT the principal driving force in early cellular evolution. Primitive cells did not carry a stable organismal genealogical trace. Primitive cellular evolution is basically communal. The high level of novelty required to evolve cell designs is a product of communal invention, of the universal HGT field, not intralineage variation. It is the community as a whole, the ecosystem, which evolves. The individual cell designs that evolved in this way are nevertheless fundamentally distinct, because the initial conditions in each case are somewhat different. As a cell design becomes more complex and interconnected a critical point is reached where a more integrated cellular organization emerges, and vertically generated novelty can and does assume greater importance."

Wohlforth, C., Hendrix, A.R., 2016a, Let's Colonize Titan, Scientific American

Wohlforth, C., Hendrix, A.R., 2016b, Beyond Earth: Our Path to a New Home in the Planets, Knopf Doubleday Publishing Group

Worth, R.J., Sigurdsson, S. and House, C.H., 2013. Seeding life on the moons of the outer planets via lithopanspermia. Astrobiology, 13(12), pp.1155-1165.

Wynn-Williams, D.D., Edwards, H.G.M., Newton, E.M. and Holder, J.M., 2002. Pigmentation as a survival strategy for ancient and modern photosynthetic microbes under high ultraviolet stress on planetary surfaces. International Journal of Astrobiology, 1(1), pp.39-49.

Yarlett, N. and Hackstein, J.H., 2005. Hydrogenosomes: one organelle, multiple origins. Bioscience, 55(8), pp.657-668.

Yeoman, B., 2014. Why the passenger pigeon went extinct. Audubon, May-June. 
Yocum, R.R., Rasmussen, J.R. and Strominger, J.L., 1980. The mechanism of action of penicillin. Penicillin acylates the active site of Bacillus stearothermophilus D-alanine carboxypeptidase. Journal of Biological Chemistry, 255(9), pp.3977-3986.

Young, R.S., Painter, R.B. and Johnson, R.D., 1965. An analysis of the extraterrestrial life detection problem. NASA Special Publication, 75, "that the probability that a single viable organism be aboard any vehicle intended for planetary landing must be less than 1 in $10,000^{\prime \prime}$

Zakharova, K., Marzban, G., de Vera, J.P., Lorek, A. and Sterflinger, K., 2014. Protein patterns of black fungi under simulated Mars-like conditions. Scientific reports, 4, p.5114.

Zhu, S., Zhu, M., Knoll, A.H., Yin, Z., Zhao, F., Sun, S., Qu, Y., Shi, M. and Liu, H., 2016. Decimetre-scale multicellular eukaryotes from the 1.56-billion-year-old Gaoyuzhuang Formation in North China. Nature communications, 7, p.11500.

Zubrin, R. (2016) starring in episode 0 (making of) of season 1 of the National Geographic series Mars, 